\title{
Supporting Information for Axial Redox Tuning at a Tetragonal Cobalt Center
}

\author{
Jonghoon Choi, ${ }^{\mathrm{a}}$ Sun Hee Kim, ${ }^{\mathrm{b}}$ and Yunho Lee*a

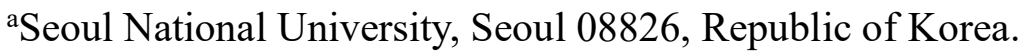 \\ ${ }^{b}$ Western Seoul Center, Korea Basic Science Institute, Seoul 03759, Republic of Korea. \\ yunhochem@snu.ac.kr
}

\section{Experimental Section}

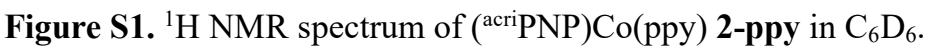

Figure S2. ${ }^{1} \mathrm{H}$ NMR spectrum of ( ${ }^{\text {acripNP) }} \mathrm{Co}(\mathrm{bzq})$ 2-bzq in $\mathrm{C}_{6} \mathrm{D}_{6}$.

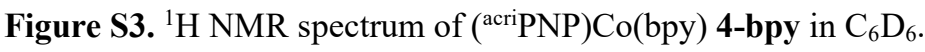

Figure S4. ${ }^{13} \mathrm{C}$ NMR spectrum of ( ${ }^{\text {acripNP) }} \mathrm{Co}(\mathrm{bpy})$ 4-bpy in $\mathrm{C}_{6} \mathrm{D}_{6}$.

Figure S5. ${ }^{31} \mathrm{P}$ NMR spectrum of ( ${ }^{\text {acripNP) }} \mathrm{Co}$ (bpy) 4-bpy in $\mathrm{C}_{6} \mathrm{D}_{6}$.

Figure S6. ${ }^{1} \mathrm{H}$ NMR spectrum of ( ${ }^{\text {acripNP) }} \mathrm{Co}$ (phen) 4-phen in $\mathrm{C}_{6} \mathrm{D}_{6}$.

Figure S7. ${ }^{13} \mathrm{C}$ NMR spectrum of ( ${ }^{\text {acripNP) }} \mathrm{Co}$ (phen) 4-phen in $\mathrm{C}_{6} \mathrm{D}_{6}$.

Figure S8. ${ }^{31} \mathrm{P}$ NMR spectrum of ( ${ }^{\text {acripNP) }} \mathrm{Co}\left(\right.$ phen) 4 -phen in $\mathrm{C}_{6} \mathrm{D}_{6}$.

Figure S9. ${ }^{1} \mathrm{H}$ NMR spectrum of $\left\{\left({ }^{\text {acri }} \mathrm{PNP}\right) \mathrm{Co}(\mathrm{bpy})\right\}\left\{\mathrm{NTf}_{2}\right\}$ 5-bpy in $\mathrm{CD}_{3} \mathrm{CN}$.

Figure S10. ${ }^{1} \mathrm{H}$ NMR spectrum of $\left\{\left({ }^{\text {acripNP}}\right) \mathrm{Co}(\right.$ phen $\left.)\right\}\left\{\mathrm{NTf}_{2}\right\}$ 5-phen in $\mathrm{CD}_{3} \mathrm{CN}$.

Figure S11. ${ }^{1} \mathrm{H}$ NMR spectrum of ( ${ }^{\text {acriPNP) }} \mathrm{Co}(\mathrm{biph}) 6$ in $\mathrm{C}_{6} \mathrm{D}_{6}$.

Figure S12. ${ }^{13} \mathrm{C}$ NMR spectrum of (acripNP)Co(biph) 6 in $\mathrm{C}_{6} \mathrm{D}_{6}$.

Figure S13. ${ }^{31} \mathrm{P}$ NMR spectrum of ( ${ }^{\text {acripNP) }} \mathrm{Co}(\mathrm{biph}) 6$ in $\mathrm{C}_{6} \mathrm{D}_{6}$.

Figure S14. ${ }^{1} \mathrm{H}$ NMR spectrum of $\left\{\left({ }^{\text {acriPNP }}\right) \mathrm{Co}(\mathrm{ppy})\right\}\left\{\mathrm{NTf}_{2}\right\}$ 7-ppy in THF- $d_{8}$.

Figure S15. ${ }^{13} \mathrm{C}$ NMR spectrum of $\left\{\left({ }^{\text {acriPNP }}\right) \mathrm{Co}(\mathrm{ppy})\right\}\left\{\mathrm{NTf}_{2}\right\}$ 7-ppy in THF- $d_{8}$.

Figure S16. ${ }^{31} \mathrm{P}$ NMR spectrum of $\left\{\left({ }^{\text {acriPNP}}\right) \mathrm{Co}(\mathrm{ppy})\right\}\left\{\mathrm{NTf}_{2}\right\}$ 7-ppy in THF- $d_{8}$.

Figure S17. ${ }^{1} \mathrm{H}$ NMR spectrum of $\left\{\left({ }^{\text {acriPNP }}\right) \mathrm{Co}(\right.$ bzq $\left.)\right\}\left\{\mathrm{NTf}_{2}\right\}$ 7-bzq in THF- $d_{8}$.

Figure S18. ${ }^{13} \mathrm{C}$ NMR spectrum of $\left\{\left({ }^{\text {acriPNP }}\right) \mathrm{Co}(\mathrm{bzq})\right\}\left\{\mathrm{NTf}_{2}\right\}$ 7-bzq in THF- $d_{8}$.

Figure S19. ${ }^{31} \mathrm{P}$ NMR spectrum of $\left\{\left({ }^{\text {acriPNP}}\right) \mathrm{Co}(\mathrm{bzq})\right\}\left\{\mathrm{NTf}_{2}\right\}$ 7-bzq in THF- $d_{8}$.

Figure S20. ${ }^{1} \mathrm{H}$ NMR spectrum of $\left\{\left({ }^{\text {acripNP }}\right) \mathrm{Co}(\mathrm{biph})\right\}\{\mathrm{K}(18-\mathrm{C}-6)\} \mathbf{8}$ in $\mathrm{CD}_{3} \mathrm{CN}$.

Figure S21. ${ }^{1} \mathrm{H}$ NMR spectra of the reaction of 6 with (a) $\mathrm{CoCp} 2$ and $\mathrm{CoCp}{ }_{2}$ in $\mathrm{THF} / \mathrm{C}_{6} \mathrm{D}_{6}$.

Figure S22. ${ }^{31} \mathrm{P}$ NMR spectra of the reaction of 6 with (a) $\mathrm{CoCp} \mathrm{p}_{2}$ and $\mathrm{CoCp}{ }_{2}$ in $\mathrm{THF} / \mathrm{C}_{6} \mathrm{D}_{6}$.

Figure S23. Solid-state structure of 2-ppy.

Table S1. Selected bond distances and angles for 2-ppy.

Figure S24. Solid-state structure of 2-bzq.

Table S2. Selected bond distances and angles for 2-bzq.

Figure S25. Solid-state structure of 4-bpy.

Table S3. Selected bond distances and angles for 4-bpy.

Figure S26. Solid-state structure of 4-phen.

Table S4. Selected bond distances and angles for 4-phen.

Figure S27. Solid-state structure of 5-bpy.

Table S5. Selected bond distances and angles for 5-bpy.

Figure S28. Solid-state structure of 5-phen.

Table S6. Selected bond distances and angles for 5-phen.

Figure S29. Solid-state structure of 6.

Table S7. Selected bond distances and angles for 6 .

Figure S30. Solid-state structure of 7-ppy. 
Table S8. Selected bond distances and angles for 7-ppy.

Figure S31. Solid-state structure of 7-bzq.

Table S9. Selected bond distances and angles for 7-bzq.

Figure S32. Solid-state structure of $\mathbf{8}$.

Table S10. Selected bond distances and angles for $\mathbf{8}$.

Figure S33. Cobalt centered core structures of 4-bpy, 5-bpy, 4-phen and 5-phen.

Figure S34. Nitrogen centered core structures of 4-bpy, 5-bpy, 4-phen and 5-phen.

Figure S35. Structural comparison of bidentate ligands in (a) 4-bpy and (b) 4-phen.

Figure S36. UV-Vis spectra of 2-ppy and 2-bzq.

Figure S37. UV-Vis spectra of 4-bpy and 4-phen.

Figure S38. UV-Vis spectra of 5-bpy and 5-phen.

Figure S39. UV-Vis spectra of 6.

Figure S40. UV-Vis spectra of 7-ppy and 7-bzq.

Figure S41. UV-Vis spectra of 8 .

Figure S42. X-Band EPR spectrum of 2-ppy in toluene at $10 \mathrm{~K}$.

Figure S43. X-Band EPR spectrum of 2-bzq in toluene at $10 \mathrm{~K}$.

Figure S44. X-Band EPR spectrum of 5-bpy in toluene at $10 \mathrm{~K}$.

Figure S45. X-Band EPR spectrum of 5-phen in toluene at $10 \mathrm{~K}$.

Figure S46. X-Band EPR spectrum of 8 in toluene at $10 \mathrm{~K}$.

Figure S47. Normalized Co K-edge spectra of 2-bzq, 4-bpy, 4-phen and 6.

Figure S48. Cyclic voltammogram of 3-N2, 2-ppy, 2-bzq, 4-bpy, 4-phen and 6.

Figure S49. Cyclic voltammogram of 2-ppy after 1st, 50th and 100th cycles.

Figure S50. Cyclic voltammogram of 2-bzq after 1st, 50th and 100th cycles.

Figure S51. Cyclic voltammogram of 4-bpy after 1st, 50th and 100th cycles.

Figure S52. Cyclic voltammogram of 4-phen after 1st, 50th and 100th cycles.

Figure S53. Cyclic voltammogram of $\mathbf{6}$ after 1st, 50th and 100th cycles.

Figure S54. Cyclic voltammogram of 2-ppy in the presence of fc after 1st, 50th and 100th cycles.

Figure S55. Cyclic voltammogram of 4-bpy in the presence of fe after 1st, 50th and 100th cycles.

Figure S56. Cyclic voltammogram of 6 in the presence of fc after 1st, 50th and 100th cycles.

Table S11. Solvent evaporation effect over the CV cycling test of 4-bpy in the presence of Fc; current and relative current over cycles.

Table S12. Solvent evaporation effect over the CV cycling test of $\mathbf{6}$ in the presence of Fc; current and relative current over cycles.

Table S13. Peak-current ratio $\left(i_{\mathrm{p} a} / \mathrm{i}_{\mathrm{pc}}\right)$ acquired from Figure S50 and Figure S52.

Figure S57. Unrestricted open shell calculation for Electronic structures for 2-ppy.

Figure S58. Unrestricted open shell calculation for Electronic structures for 2-ppy'.

Figure S59. Unrestricted open shell calculation for Electronic structures for 2-bzq.

Figure S60. Unrestricted open shell calculation for Electronic structures for 2-bzq'.

Figure S61. Restricted open shell calculation for Electronic structure for 2-ppy and 2-bzq.

Table S14. Computed energies for 2-ppy, 2-bzq, 2-ppy' and 2-bzq'.

Figure S62. Electronic structure for the open shell singlet state of 2-ppy ${ }^{+}$.

Figure S63. Electronic structure for the triplet state of 2-ppy $\mathbf{y}^{+}$.

Figure S64. Electronic structure for the closed shell singlet state of 2-ppy $\mathbf{y}^{+}$and $\mathbf{2}-\mathbf{b z q}^{+}$.

Figure S65. Electronic structure for the open shell singlet state of 2-bzq $\mathbf{~}^{+}$.

Figure S66. Electronic structure for the triplet state of $\mathbf{2}-\mathbf{b z q}^{+}$.

Table S15. Computed energies for 2-ppy ${ }^{+}$and 2-bzq ${ }^{+}$.

Figure S67. Electronic structure for 2-ppy ${ }^{2+}$.

Figure S68. Electronic structure for 2-bzq ${ }^{2+}$

Figure S69. Electronic structure the closed shell singlet state of 2-ppy 
Figure S70. Electronic structure the triplet state of 2-ppy

Table S16. Computed energies for 2-ppy ${ }^{-}$.

Figure S71. Electronic structures for the open shell singlet state of 4-bpy.

Figure S72. Electronic structures for the triplet state of 4-bpy.

Figure S73. Electronic structures for the closed shell singlet state of 4-bpy.

Figure S74. Electronic structures for the open shell singlet state of 4-phen.

Figure S75. Electronic structures for the triplet state of 4-phen.

Figure S76. Electronic structures for the closed shell singlet state of 4-phen.

Table S17. Computed energies for 4-bpy and 4-phen.

Table S18. Mulliken spin density for the open shell singlet state of 4-bpy and 4-phen.

Figure S77. Electronic structure for 4-bpy ${ }^{+}$.

Figure S78. Electronic structure for 4-phen ${ }^{+}$.

Figure S79. Electronic structure for the open shell singlet state of 4-bpy and 4-bpy ${ }^{+}$.

Figure S80. DFT-calculated acriPNP ligand-based orbital diagram for 4-bpy and 4-bpy ${ }^{+}$.

Figure S81. Electronic structures for the open shell singlet state of 4-bpy ${ }^{2+}$.

Figure S82. Electronic structures for the triplet state of $\mathbf{4 - b p y ^ { 2 + }}$.

Figure S83. Electronic structures for the closed shell singlet state of 4-bpy ${ }^{2+}$ and 4-phen ${ }^{2+}$.

Figure S84. Electronic structures for the open shell singlet state of 4-phen ${ }^{2+}$.

Figure S85. Electronic structures for the triplet state of 4-phen ${ }^{2+}$.

Table S19. Computed energies for 4-bpy ${ }^{2+}$ and 4-phen ${ }^{2+}$.

Figure S86. Electronic structures for the doublet state of 4-bpy ${ }^{-}$.

Figure S87. Electronic structures for the quartet state of 4-bpy $\mathbf{y}^{-}$.

Table S20. Computed energies for 4-bpy ${ }^{-}$.

Figure S88. Electronic structures for $\mathbf{6}$.

Figure S89. Electronic structures for $6^{-}$.

Figure S90. Electronic structures for the closed shell singlet state of $\mathbf{6}^{2-}$.

Figure S91. Electronic structures for the triplet state of $\mathbf{6}^{\mathbf{2}}$.

Table S21. Computed energies for $\mathbf{6}^{2-}$. 
Figure S1. ${ }^{1} \mathrm{H}$ NMR spectrum of (acripNP)Co(ppy) (2-ppy) in $\mathrm{C}_{6} \mathrm{D}_{6}$ at room temperature.

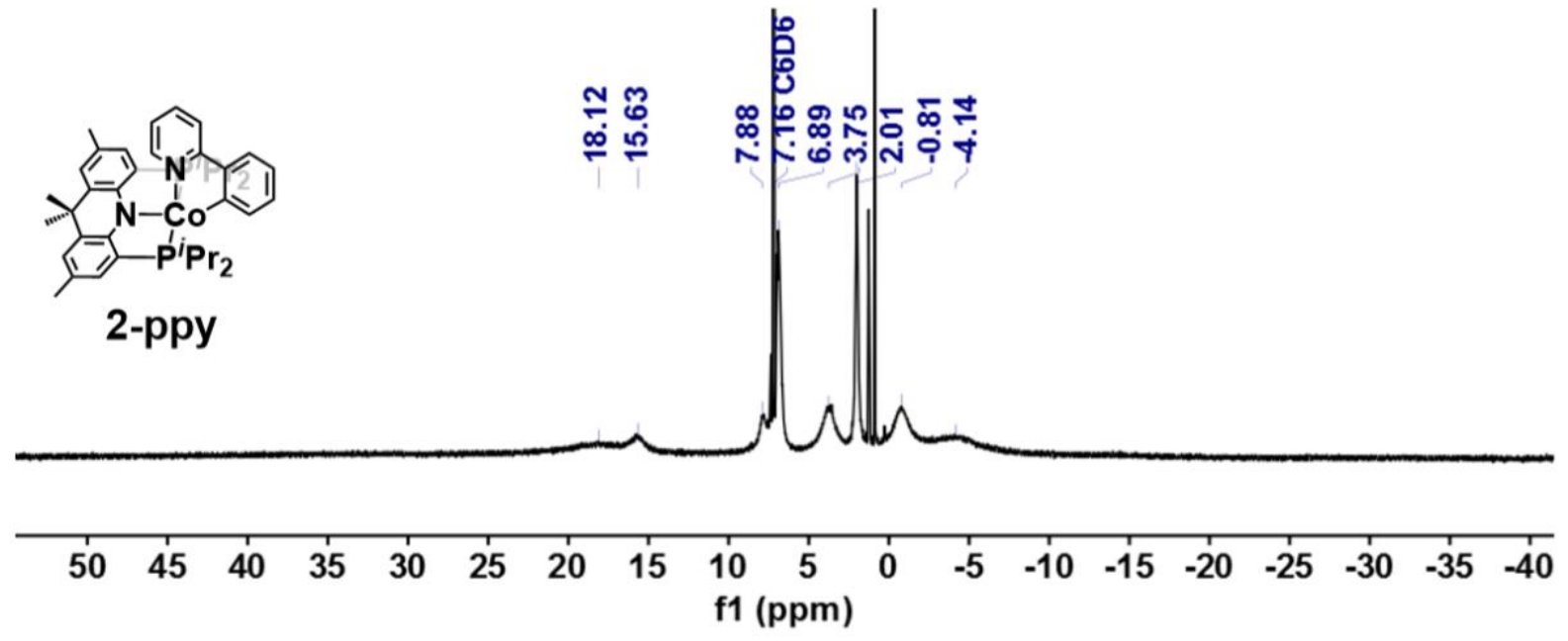

Figure S2. ${ }^{1} \mathrm{H}$ NMR spectrum of (acripNP)Co(bzq) (2-bzq) in $\mathrm{C}_{6} \mathrm{D}_{6}$ at room temperature.
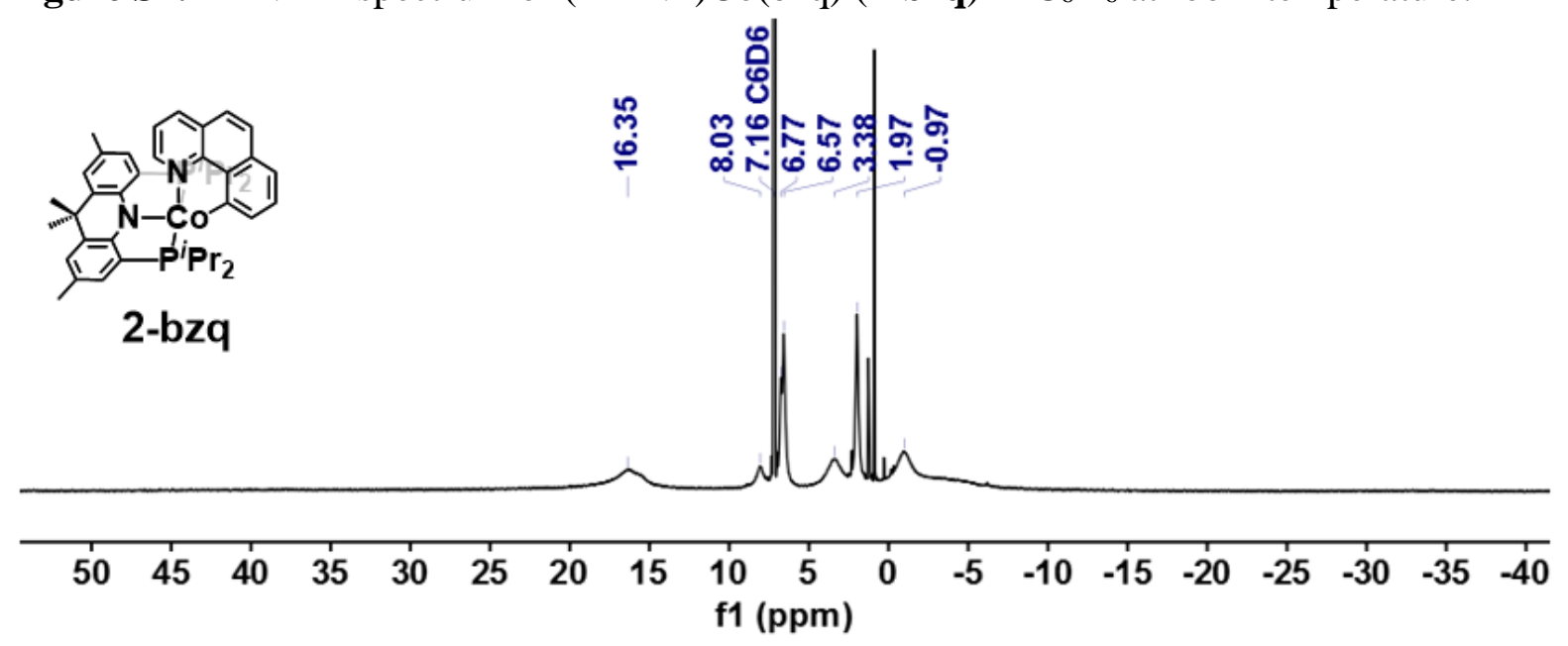

Figure S3. ${ }^{1} \mathrm{H}$ NMR spectrum of (acripNP)Co(bpy) (4-bpy) in $\mathrm{C}_{6} \mathrm{D}_{6}$ at room temperature.

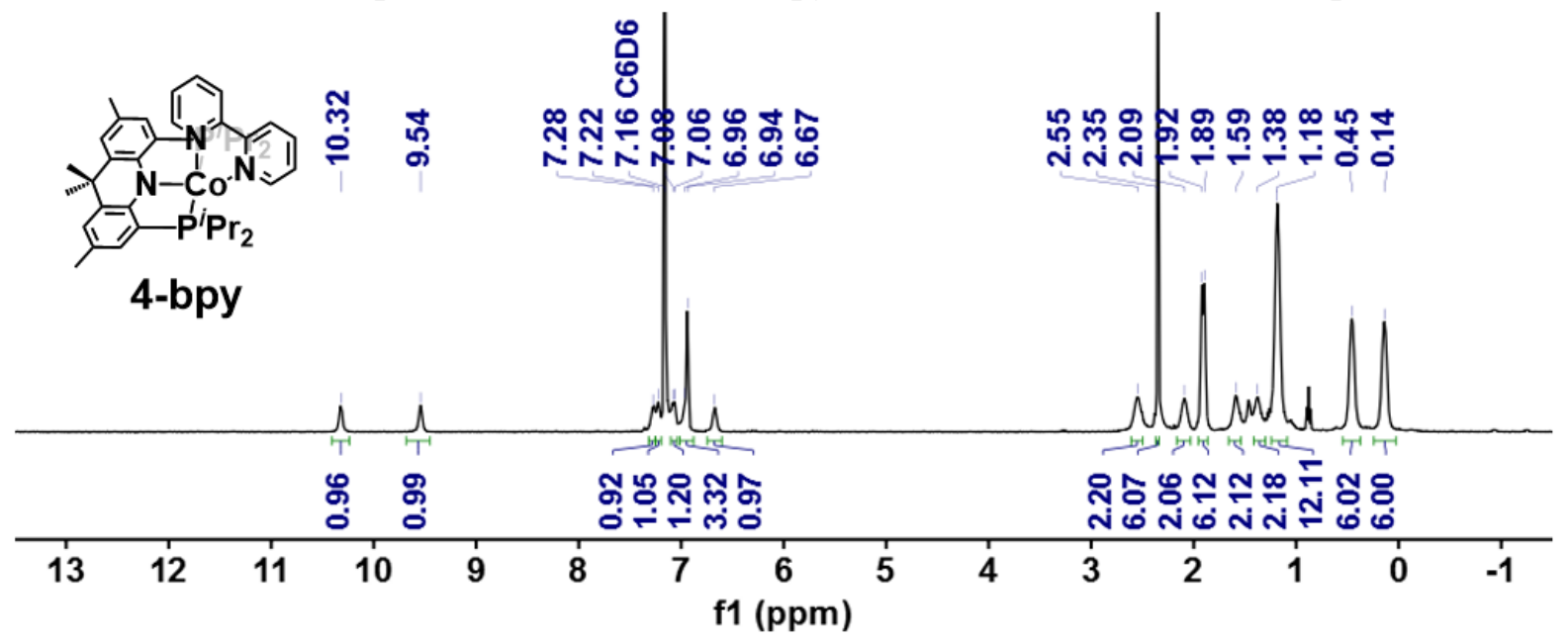


Figure S4. ${ }^{13} \mathrm{C}$ NMR spectrum of (acripNP)Co(bpy) (4-bpy) in $\mathrm{C}_{6} \mathrm{D}_{6}$ at room temperature.

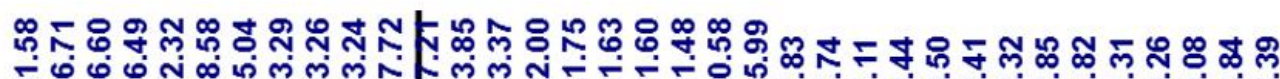

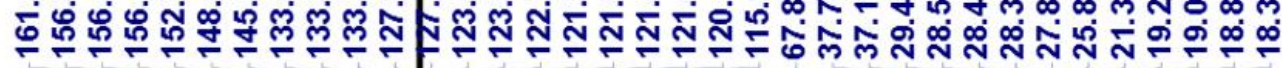

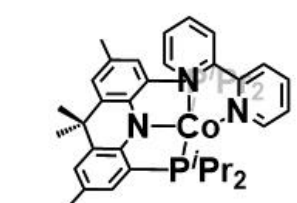

4-bpy

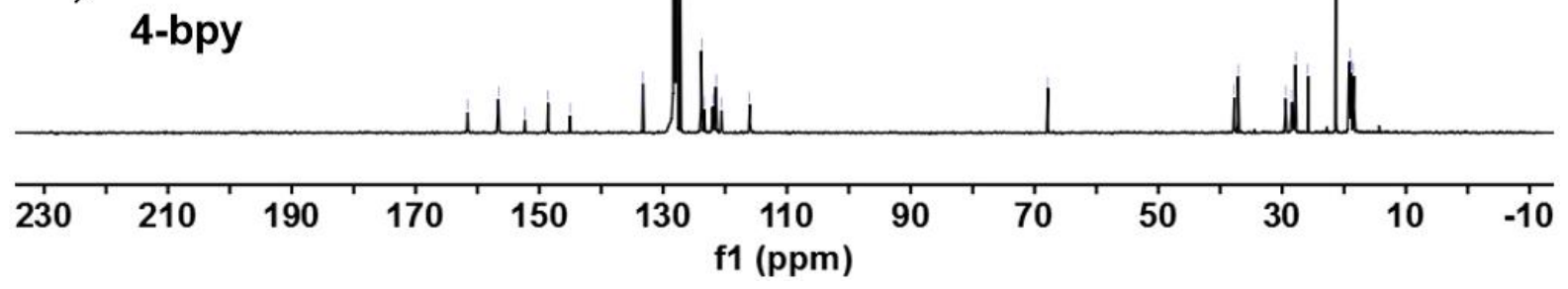

Figure S5. ${ }^{31} \mathrm{P}$ NMR spectrum of (acripNP)Co(bpy) (4-bpy) in $\mathrm{C}_{6} \mathrm{D}_{6}$ at room temperature.

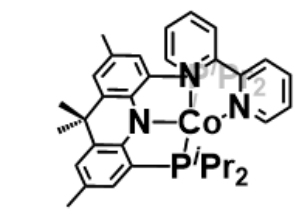

๑ొర

ถุ่

4-bpy

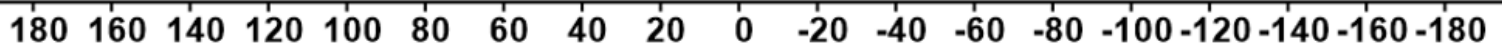
f1 (ppm)

Figure S6. ${ }^{1} \mathrm{H}$ NMR spectrum of (acripNP)Co(phen) (4-phen) in $\mathrm{C}_{6} \mathrm{D}_{6}$ at room temperature.

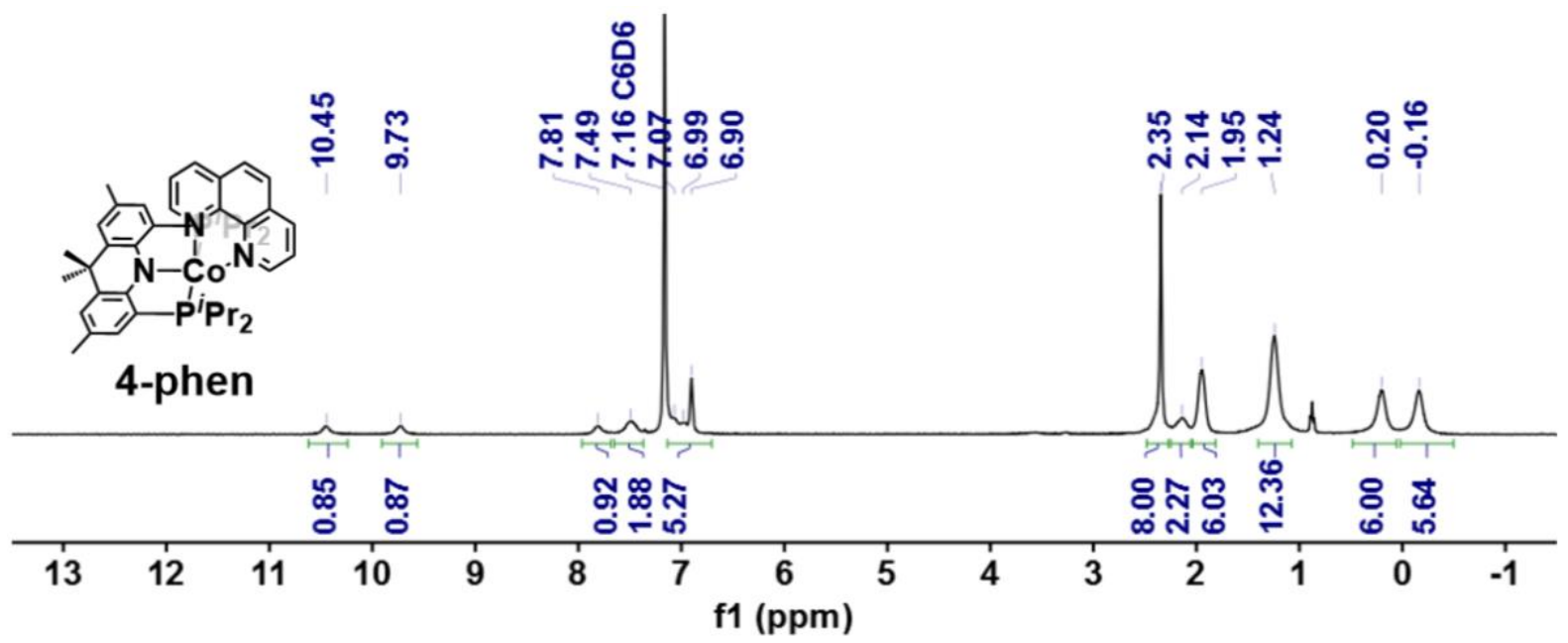


Figure S7. ${ }^{13} \mathrm{C}$ NMR spectrum of (acripNP)Co(phen) (4-phen) in $\mathrm{C}_{6} \mathrm{D}_{6}$ at room temperature.

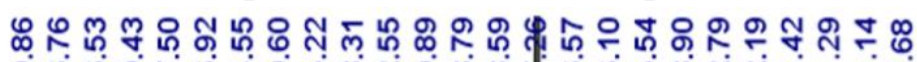

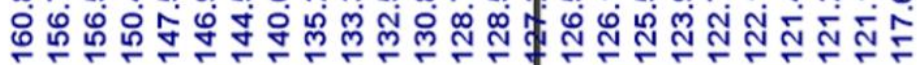

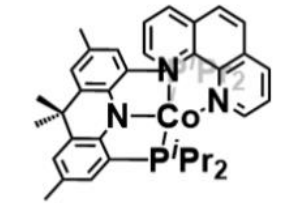

4-phen

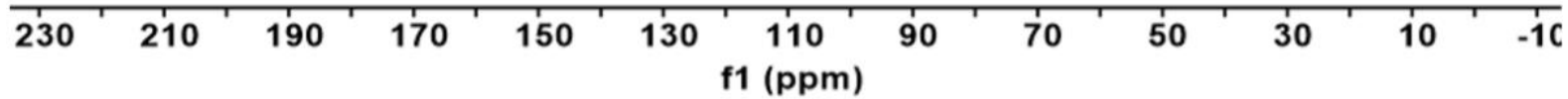

Figure S8. ${ }^{31} \mathrm{P}$ NMR spectrum of (acripNP)Co(phen) (4-phen) in $\mathrm{C}_{6} \mathrm{D}_{6}$ at room temperature.

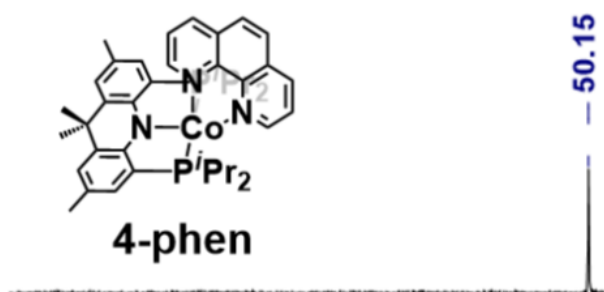

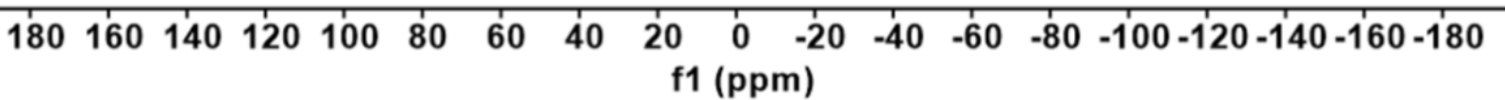

Figure S9. ${ }^{1} \mathrm{H}$ NMR spectrum of $\left\{\left({ }^{\text {acripNP}}\right) \mathrm{Co}(\right.$ bpy $\left.)\right\}\left\{\mathrm{NTF}_{2}\right\}$ (5-bpy) in $\mathrm{CD}_{3} \mathrm{CN}$ at room temperature.

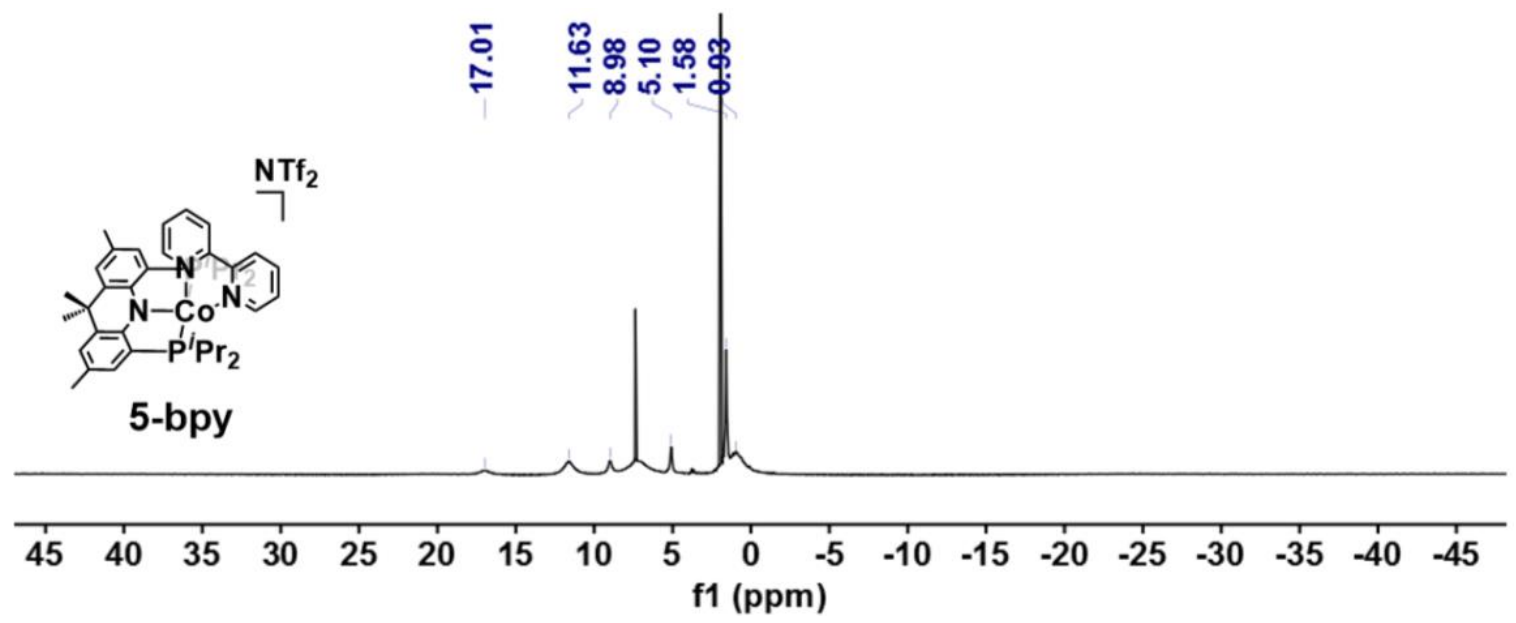


Figure S10. ${ }^{1} \mathrm{H}$ NMR spectrum of $\left\{\left({ }^{\text {acripNP}}\right) \mathrm{Co}(\mathrm{phen})\right\}\left\{\mathrm{NTF}_{2}\right\}\left(5\right.$-phen) in $\mathrm{CD}_{3} \mathrm{CN}$ at room temperature.

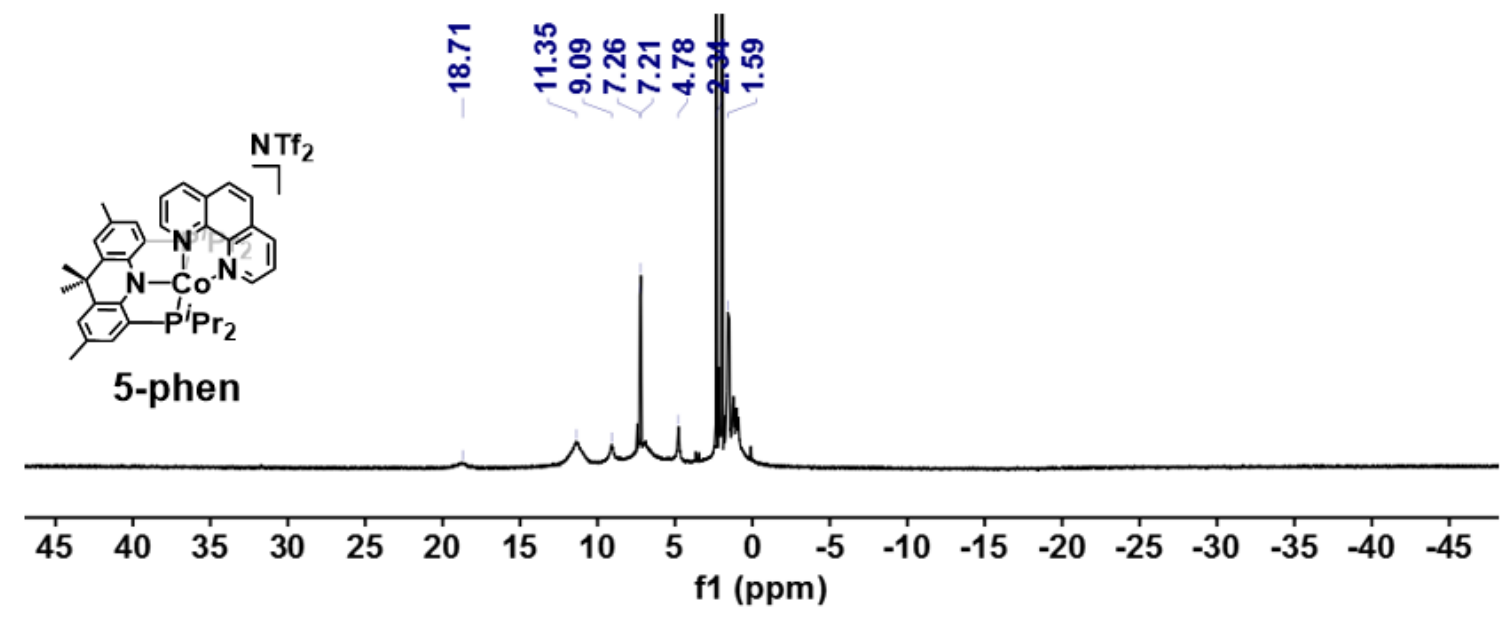

Figure S11. ${ }^{1} \mathrm{H}$ NMR spectrum of (acriPNP)Co(biph) (6) in $\mathrm{C}_{6} \mathrm{D}_{6}$ at room temperature.

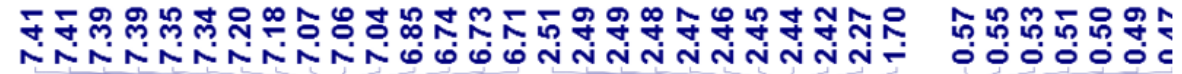
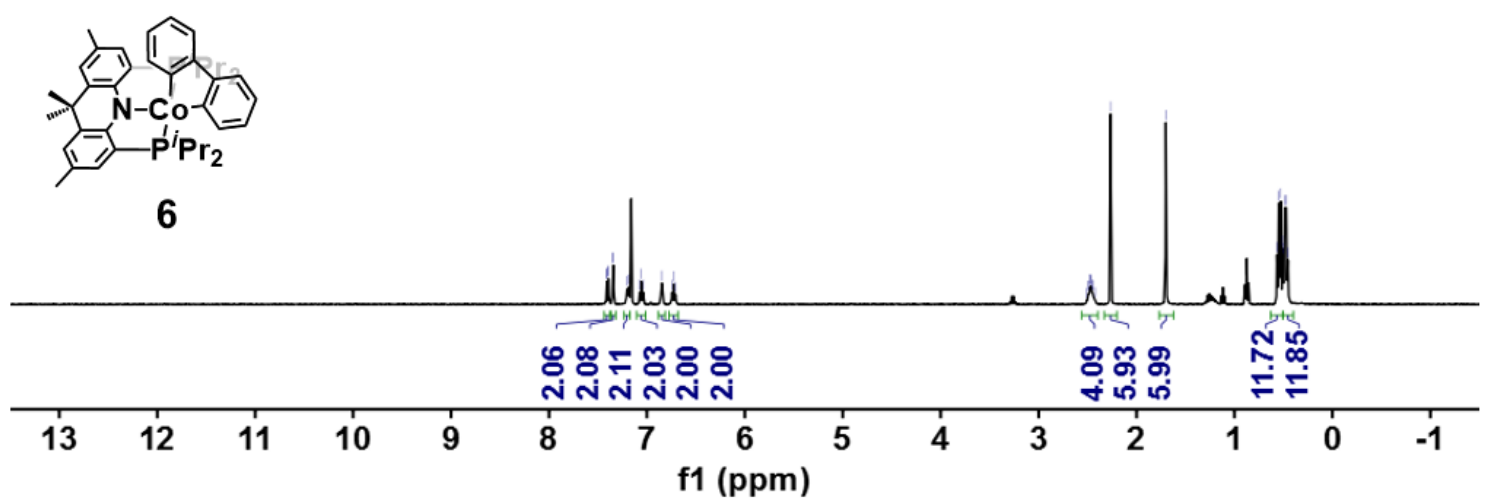

Figure S12. ${ }^{13} \mathrm{C}$ NMR spectrum of (acriPNP)Co(biph) (6) in $\mathrm{C}_{6} \mathrm{D}_{6}$ at room temperature.

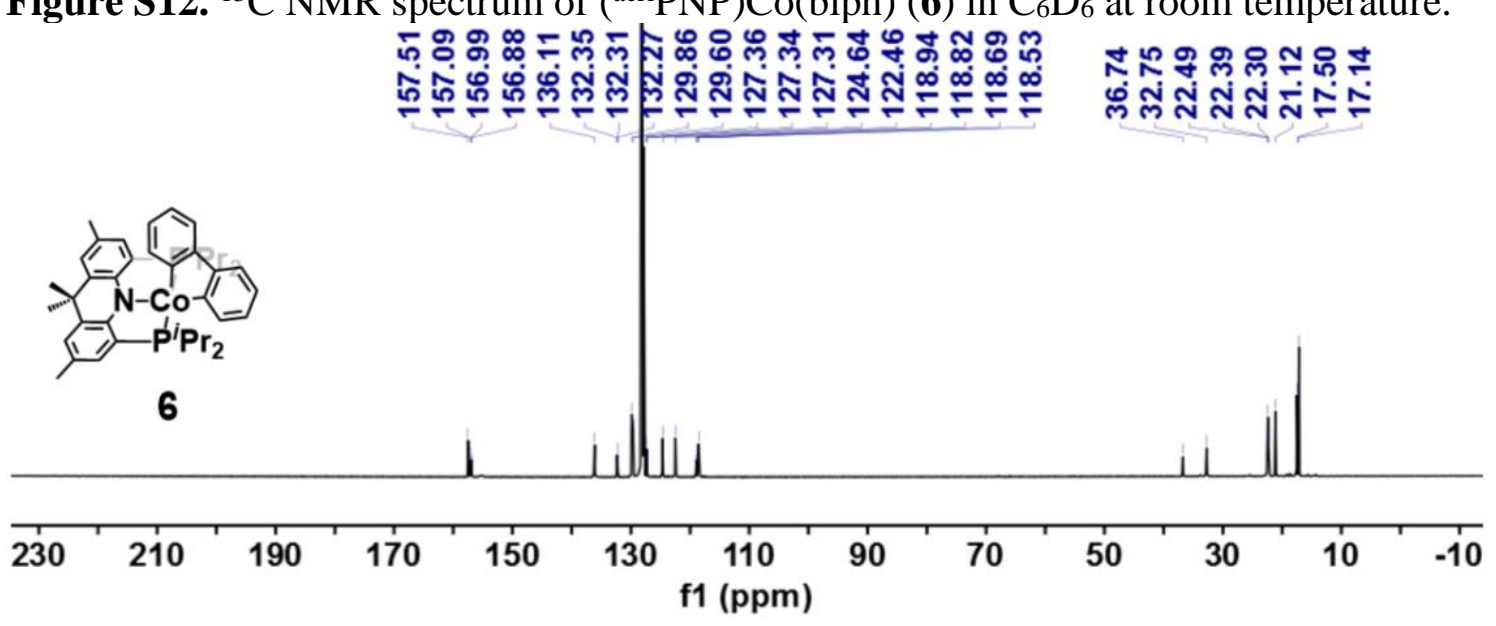


Figure S13. ${ }^{31} \mathrm{P}$ NMR spectrum of ( ${ }^{\text {acripNP)}} \mathrm{Co}(\mathrm{biph})(6)$ in $\mathrm{C}_{6} \mathrm{D}_{6}$ at room temperature.

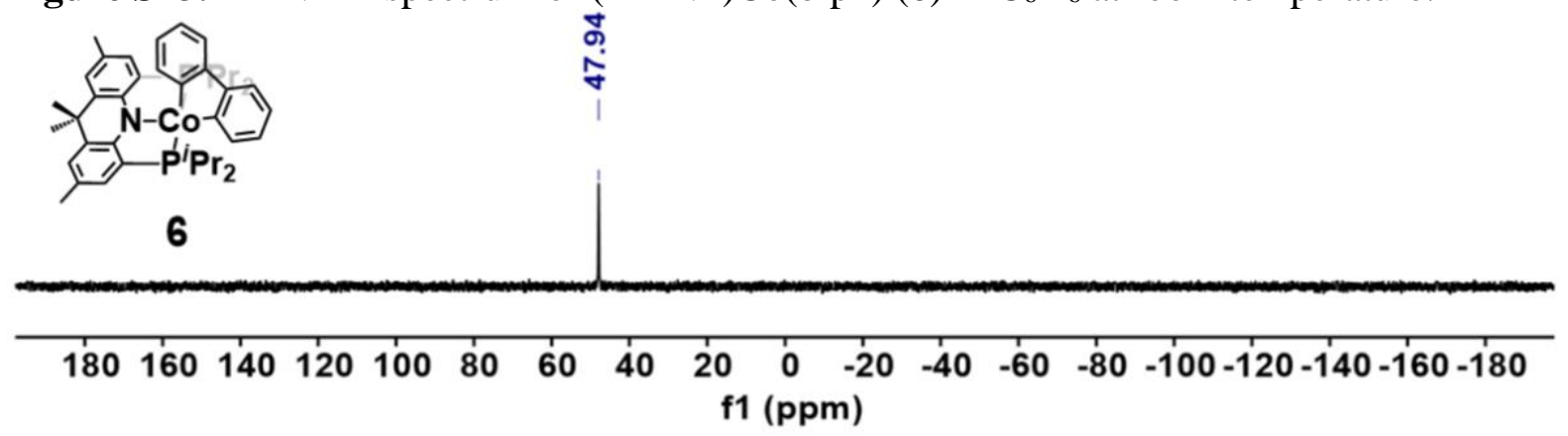

Figure S14. ${ }^{1} \mathrm{H}$ NMR spectrum of $\left\{\left({ }^{\text {acripNP) }}\right) \mathrm{Co}(\mathrm{ppy})\right\}\left\{\mathrm{NTf}_{2}\right\}$ (7-ppy) in THF- $d_{8}$ at room temperature.

\section{至}

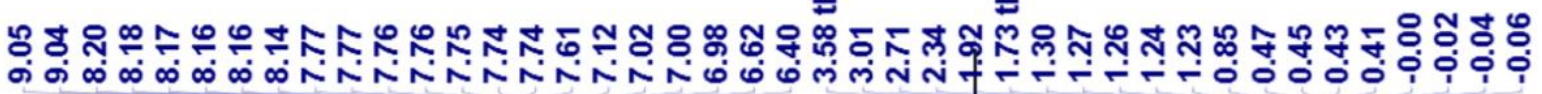

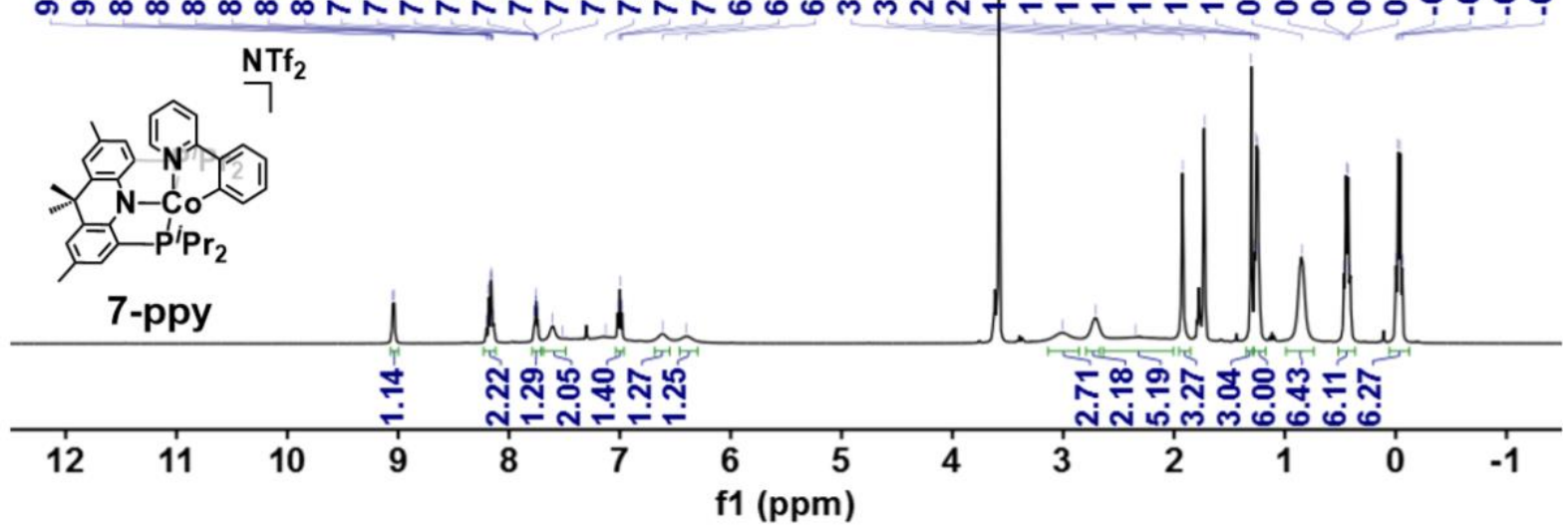

Figure S15. ${ }^{13} \mathrm{C}$ NMR spectrum of $\left\{(\right.$ acriPNP)Co(ppy) $\}\left\{\mathrm{NTf}_{2}\right\}$ (7-ppy) in THF- $d 8$ at room temperature.

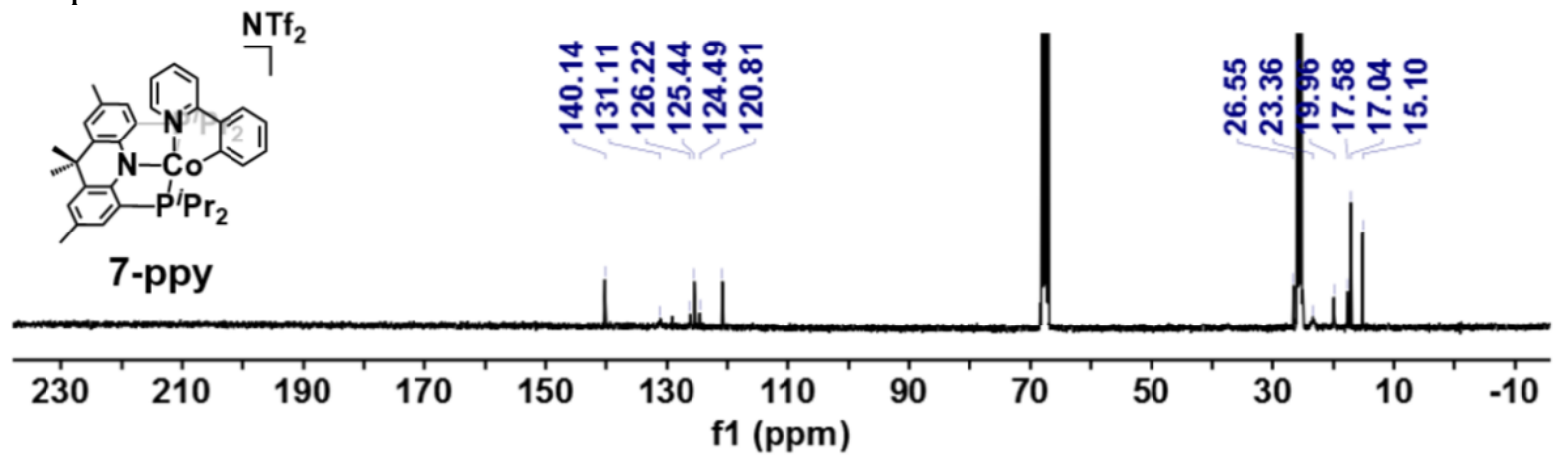


Figure S16. ${ }^{31} \mathrm{P}$ NMR spectrum of $\left\{\left({ }^{\text {acripNP}}\right) \mathrm{Co}(\mathrm{ppy})\right\}\left\{\mathrm{NTf}_{2}\right\}$ (7-ppy) in THF- $d 8$ at room temperature.

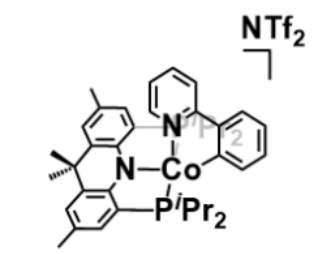

7-ppy

\section{ํำ}

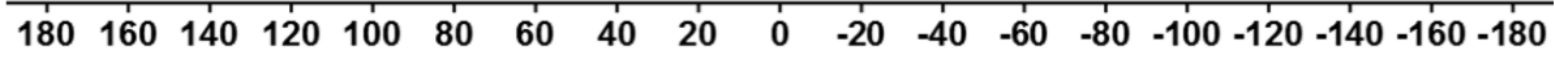 f1 (ppm)}

Figure S17. ${ }^{1} \mathrm{H}$ NMR spectrum of $\left\{\left({ }^{\text {acripNP }}\right) \mathrm{Co}(\mathrm{bzq})\right\}\left\{\mathrm{NTf}_{2}\right\}($ 7-bzq) in THF- $d 8$ at room temperature.

\section{焉焉}

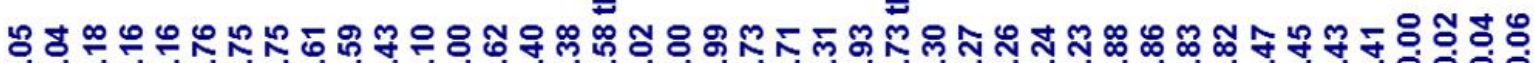
बi

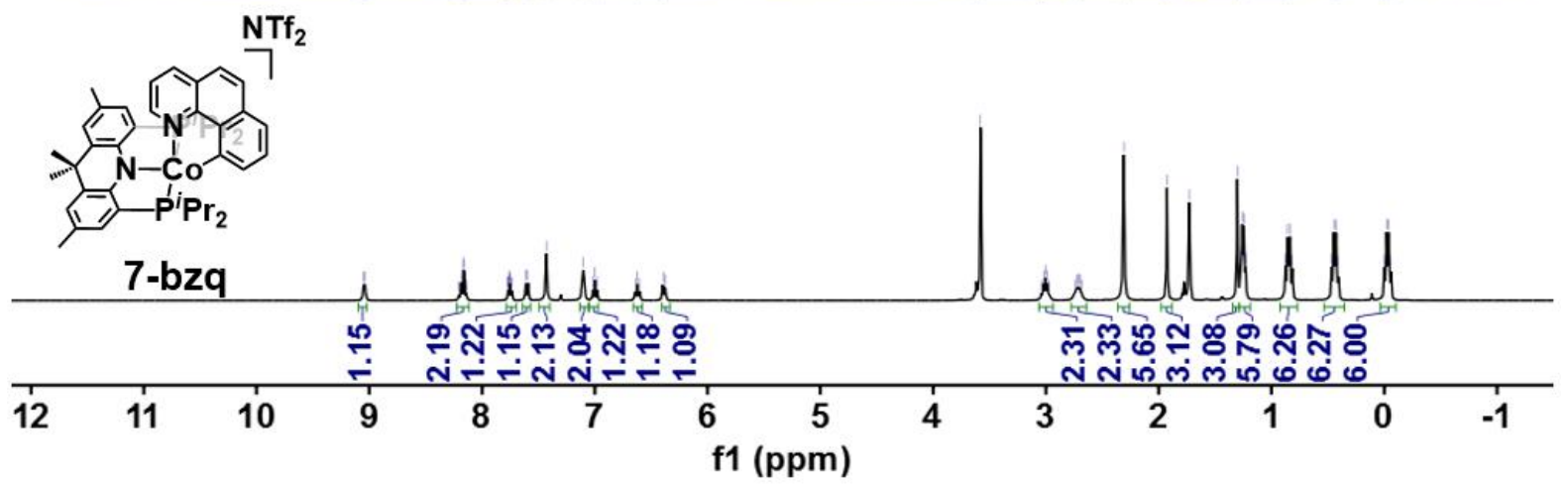

Figure S18. ${ }^{13} \mathrm{C}$ NMR spectrum of $\left\{\left({ }^{\text {acripNP) }}\right) \mathrm{Co}(\mathrm{bzq})\right\}\left\{\mathrm{NTf}_{2}\right\}$ (7-bzq) in THF- $d_{8}$ at room temperature.

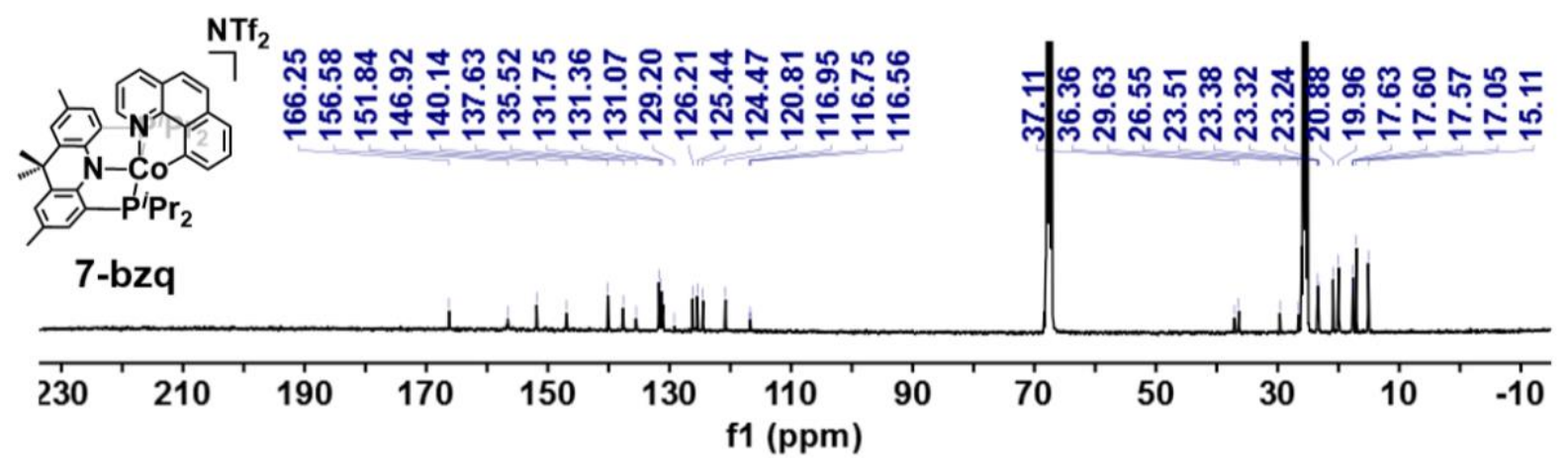


Figure S19. ${ }^{31} \mathrm{P}$ NMR spectrum of $\left\{\left({ }^{\text {acripNP}}\right) \mathrm{Co}(\mathrm{bzq})\right\}\left\{\mathrm{NTf}_{2}\right\}$ (7-bzq) in THF- $d_{8}$ at room temperature.

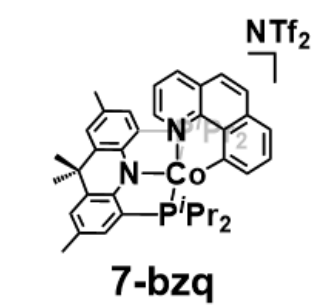

$\stackrel{\infty}{\stackrel{\infty}{\leftrightarrow}}$

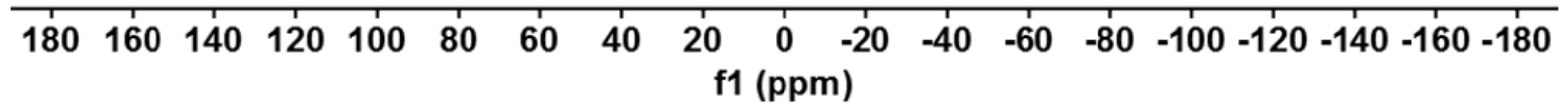

Figure S20. ${ }^{1} \mathrm{H}$ NMR spectrum of $\left\{\left({ }^{\text {acripNP }}\right) \mathrm{Co}(\right.$ biph $\left.)\right\}\{\mathrm{K}(18-\mathrm{C}-6)\}(8)$ in $\mathrm{CD}_{3} \mathrm{CN}$ at room temperature.

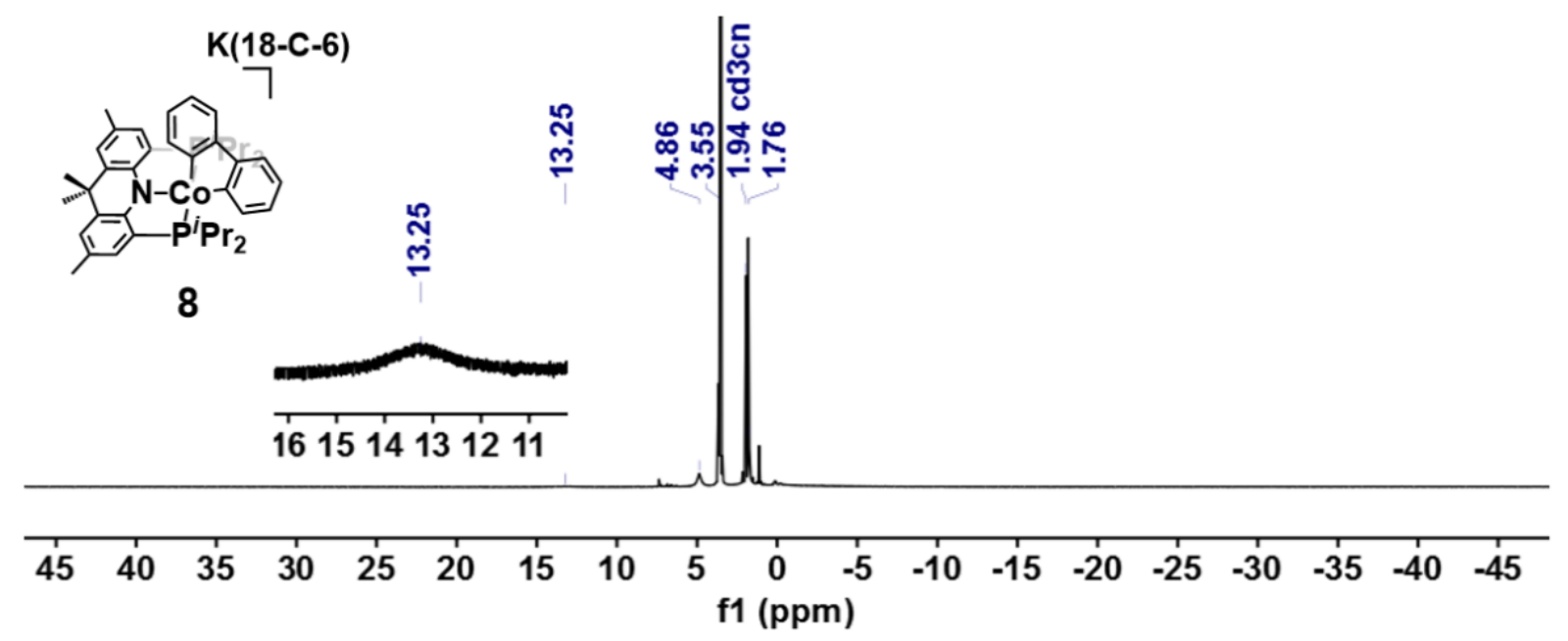


Figure S21. ${ }^{1} \mathrm{H}$ NMR spectrum of the reaction of (acriPNP)Co(biph) (6) with (a) $\mathrm{CoCp}_{2}$ and (b) $\mathrm{CoCp}_{2}{ }_{2}$ in $\mathrm{THF} / \mathrm{C}_{6} \mathrm{D}_{6}$ at room temperature $\left(\bullet: \mathrm{CoCp}_{2}, \bullet: \mathrm{CoCp}_{2}\right)$.

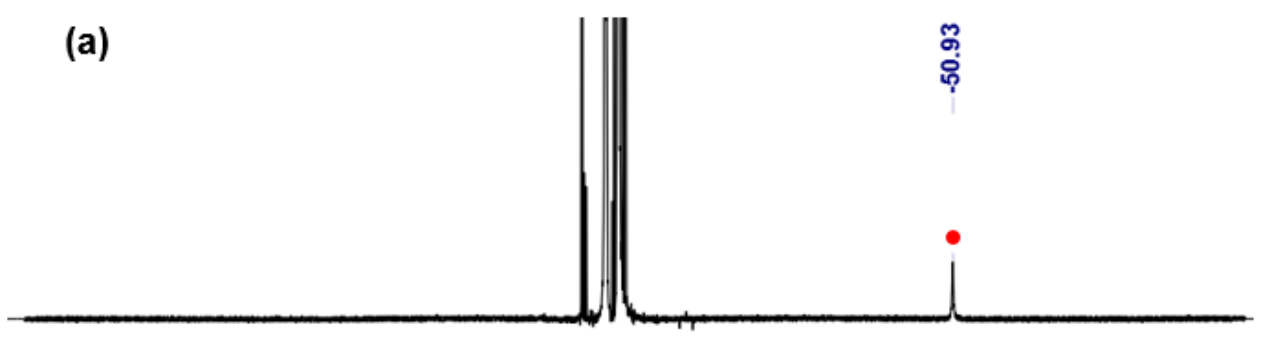

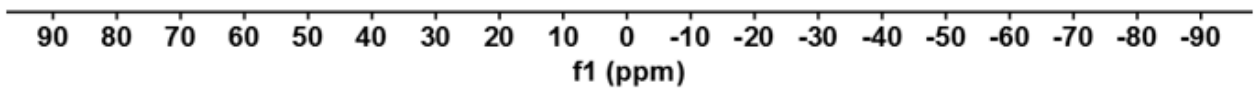

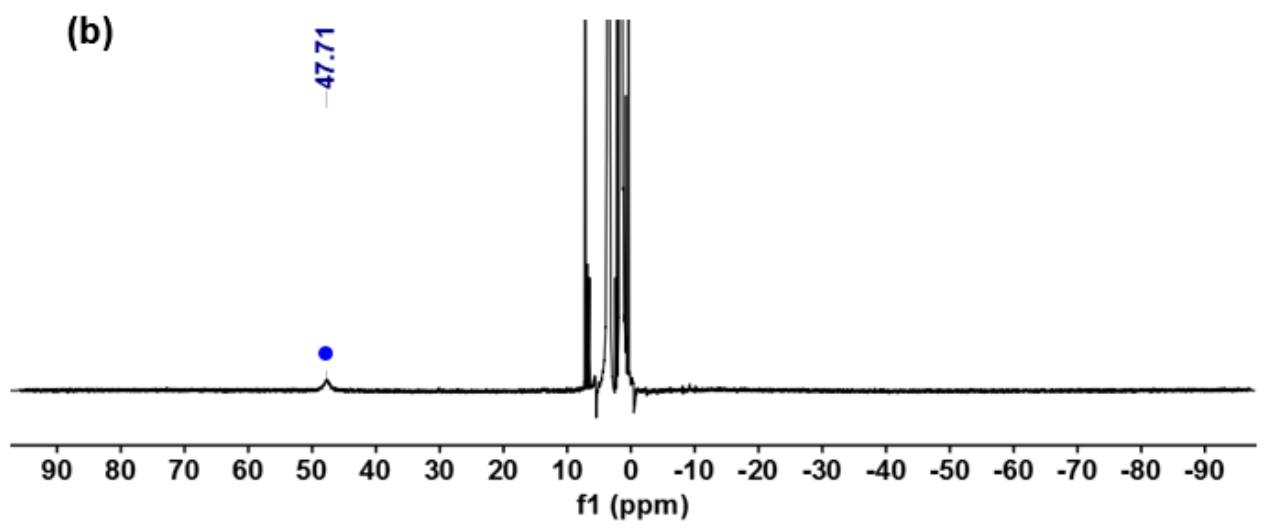

Figure S22. ${ }^{31} \mathrm{P}$ NMR spectrum of the reaction of (acripNP)Co(biph) (6) with (a) $\mathrm{CoCp}_{2}$ and (b) $\mathrm{CoCp}_{2}{ }_{2}$ in $\mathrm{THF} / \mathrm{C}_{6} \mathrm{D}_{6}$ at room temperature.

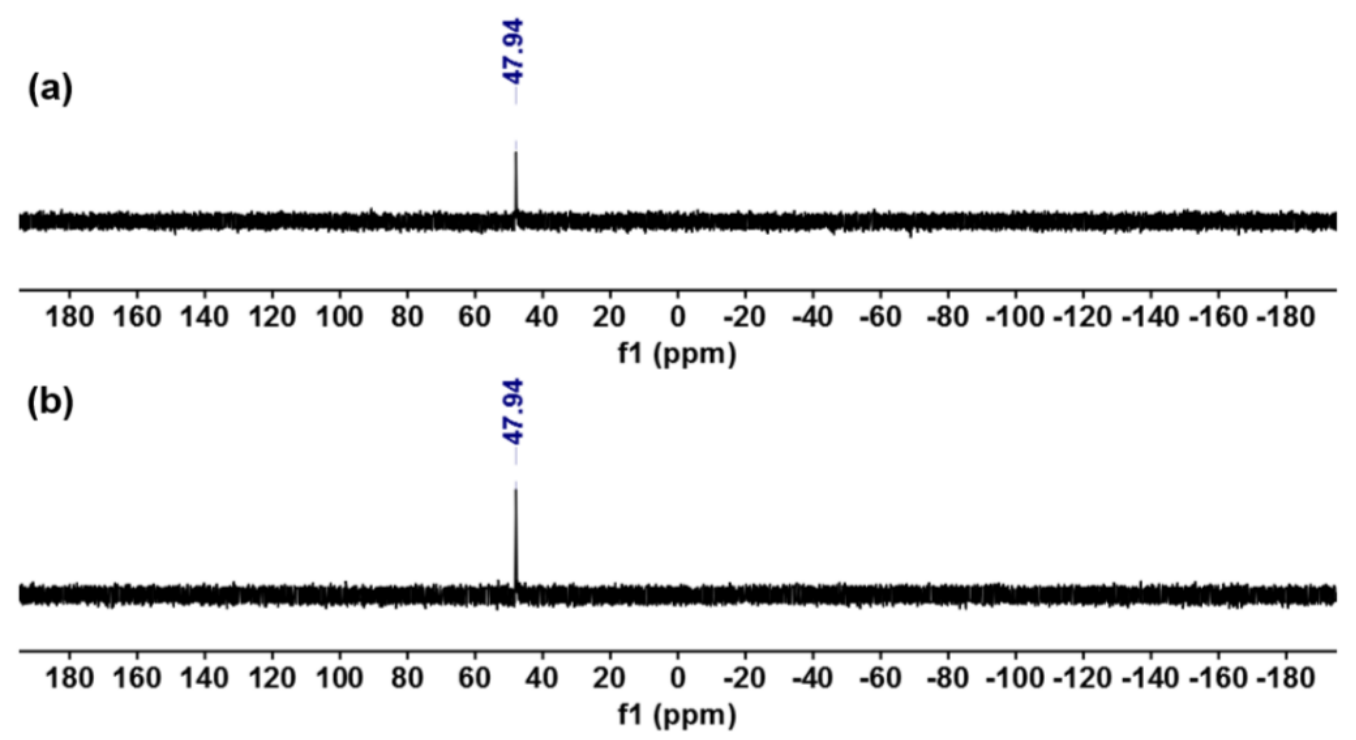


Figure S23. Solid-state structure of (acriPNP)Co(ppy) (2-ppy). All hydrogen atoms of an acripNP ligand are omitted for clarity.

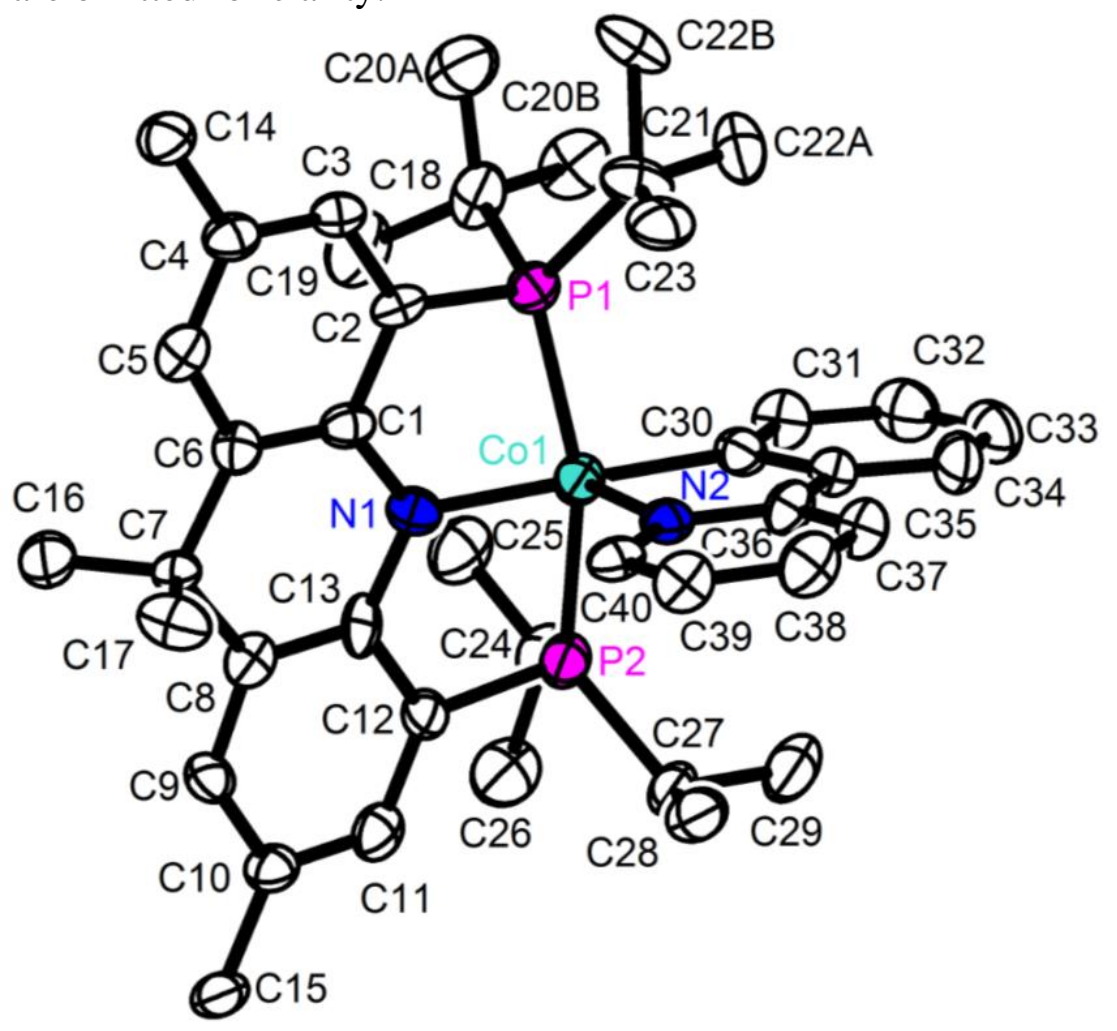

Table S1. Selected bond distances and angles for 2-ppy $\left(\AA\right.$ and $\left.{ }^{\circ}\right)$.

\begin{tabular}{cccc}
\hline \multicolumn{2}{c}{ Bond distance } & \multicolumn{2}{c}{ Bond angle } \\
\hline $\mathrm{d}_{\mathrm{Co1} 1 \mathrm{P} 1}$ & $2.210(2)$ & $\angle \mathrm{P} 1-\mathrm{Co} 1-\mathrm{P} 2$ & $158.15(7)$ \\
\hline $\mathrm{d}_{\mathrm{Co1}-\mathrm{P} 2}$ & $2.203(2)$ & $\angle \mathrm{N} 1-\mathrm{Co} 1-\mathrm{N} 2$ & $97.1(2)$ \\
\hline $\mathrm{d}_{\mathrm{Co} 1-\mathrm{N} 1}$ & $1.964(5)$ & $\angle \mathrm{N} 1-\mathrm{Co} 1-\mathrm{C} 30$ & $178.8(2)$ \\
\hline $\mathrm{d}_{\mathrm{Co1}-\mathrm{N} 2}$ & $2.016(5)$ & & \\
\hline $\mathrm{d}_{\mathrm{Co1}-\mathrm{C} 30}$ & $1.915(6)$ & & \\
\hline $\mathrm{d}_{\mathrm{N} 1-\mathrm{C} 1}$ & $1.396(7)$ & & \\
\hline $\mathrm{d}_{\mathrm{N} 1-\mathrm{C} 13}$ & $1.387(7)$ & & \\
\hline
\end{tabular}


Figure S24. Solid-state structure of (acripNP)Co(bzq) (2-bzq). All hydrogen atoms of an acriPNP ligand are omitted for clarity.

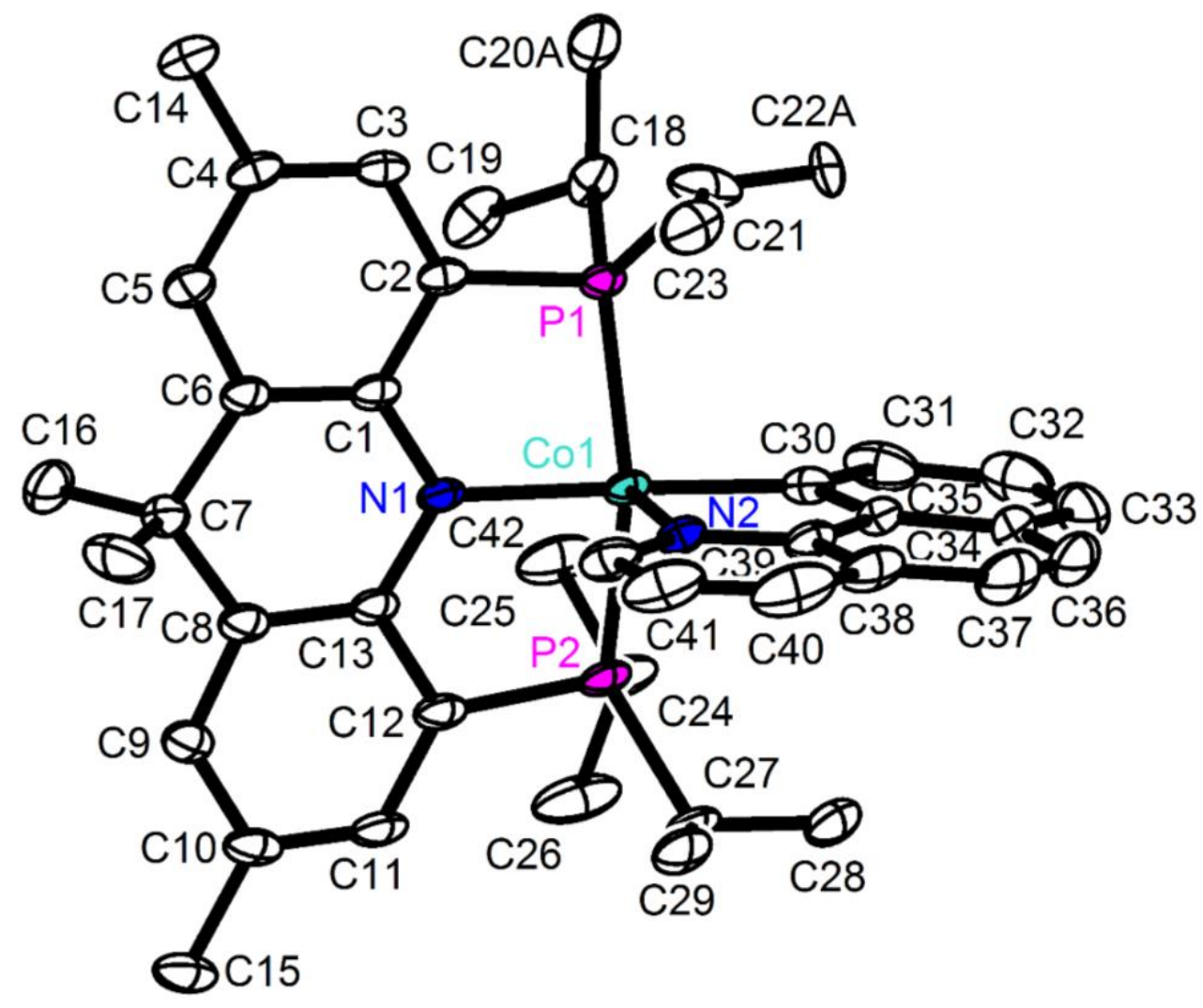

Table S2. Selected bond distances and angles for 2-bzq $\left(\AA\right.$ and $\left.{ }^{\circ}\right)$.

\begin{tabular}{cccc}
\hline \multicolumn{2}{c}{ Bond distance } & \multicolumn{2}{c}{ Bond angle } \\
\hline $\mathrm{d}_{\mathrm{C} 1-\mathrm{P} 1}$ & $2.2021(8)$ & $\angle \mathrm{P} 1-\mathrm{Co} 1-\mathrm{P} 2$ & $160.18(2)$ \\
\hline $\mathrm{d}_{\mathrm{Co1} 1 \mathrm{P} 2}$ & $2.2117(9)$ & $\angle \mathrm{N} 1-\mathrm{Co} 1-\mathrm{N} 2$ & $97.23(6)$ \\
\hline $\mathrm{d}_{\mathrm{Co} 1-\mathrm{N} 1}$ & $1.977(2)$ & $\angle \mathrm{N} 1-\mathrm{Co} 1-\mathrm{C} 30$ & $177.79(7)$ \\
\hline $\mathrm{d}_{\mathrm{Co1}-\mathrm{N} 2}$ & $2.051(1)$ & & \\
\hline $\mathrm{d}_{\mathrm{Co1} 1-\mathrm{C} 30}$ & $1.925(2)$ & & \\
\hline $\mathrm{d}_{\mathrm{N} 1-\mathrm{C} 1}$ & $1.383(2)$ & & \\
\hline $\mathrm{d}_{\mathrm{N} 1-\mathrm{C} 13}$ & $1.383(2)$ & & \\
\hline
\end{tabular}


Figure S25. Solid-state structure of (acriPNP)Co(bpy) (4-bpy). All hydrogen atoms of an acriPNP ligand are omitted for clarity.

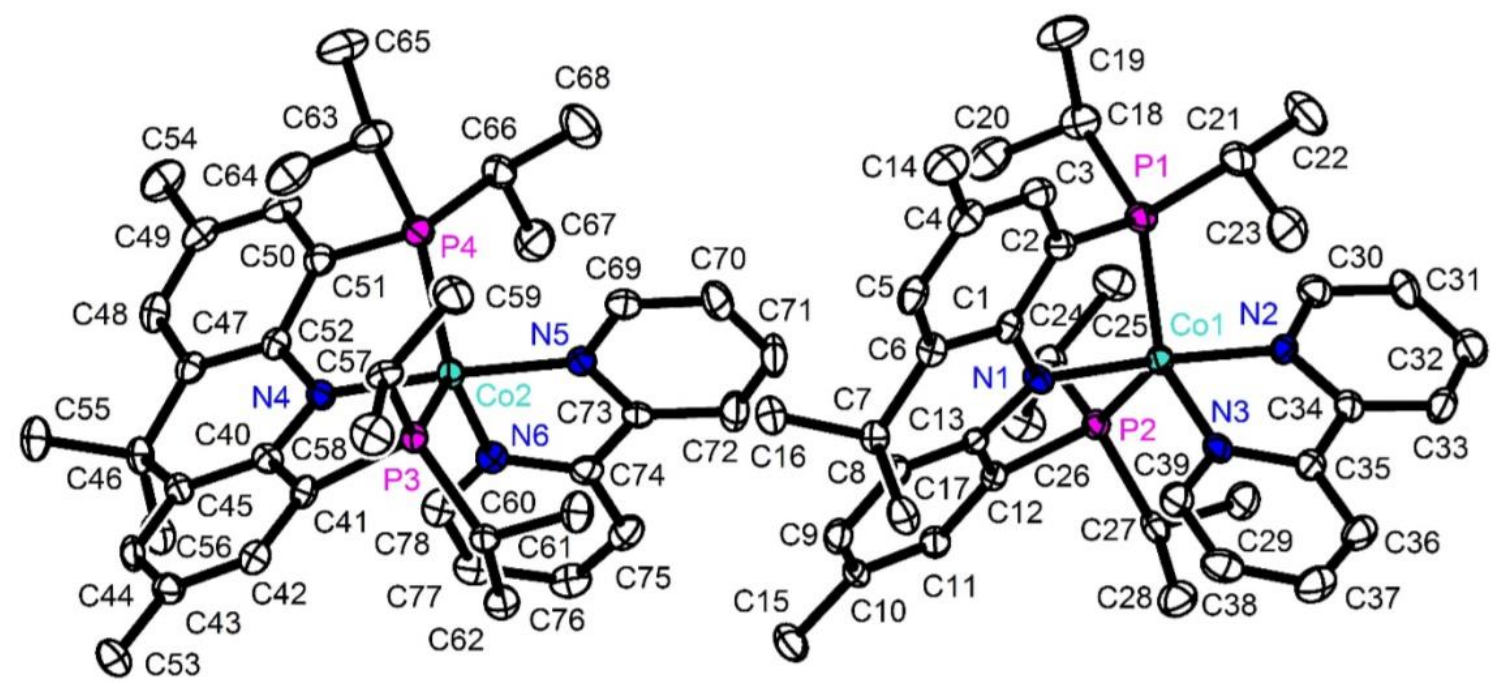

Table S3. Selected bond distances and angles for 4-bpy $\left(\AA \AA\right.$ and $\left.^{\circ}\right)$.

\begin{tabular}{cccc}
\hline \multicolumn{2}{c}{ Bond distance } & \multicolumn{2}{c}{ Bond angle } \\
\hline $\mathrm{d}_{\mathrm{Co1} 1 \mathrm{P} 1}$ & $2.224(1)$ & $\angle \mathrm{P} 1-\mathrm{Co} 1-\mathrm{P} 2$ & $129.40(4)$ \\
\hline $\mathrm{d}_{\mathrm{Co1} 1 \mathrm{P} 2}$ & $2.225(1)$ & $\angle \mathrm{N} 1-\mathrm{Co} 1-\mathrm{N} 2$ & $174.2(1)$ \\
\hline $\mathrm{d}_{\mathrm{Co1} 1 \mathrm{~N} 1}$ & $1.945(3)$ & $\angle \mathrm{N} 1-\mathrm{Co} 1-\mathrm{N} 3$ & $92.6(1)$ \\
\hline $\mathrm{d}_{\mathrm{Co1}-\mathrm{N} 2}$ & $1.903(3)$ & $\angle \mathrm{P} 1-\mathrm{Co} 1-\mathrm{N} 3$ & $115.56(9)$ \\
\hline $\mathrm{d}_{\mathrm{Co1}-\mathrm{N} 3}$ & $1.933(3)$ & $\angle \mathrm{P} 2-\mathrm{Co} 1-\mathrm{N} 3$ & $113.54(9)$ \\
\hline $\mathrm{d}_{\mathrm{Co2}-\mathrm{P} 3}$ & $2.230(1)$ & $\angle \mathrm{P} 3-\mathrm{Co} 2-\mathrm{P} 4$ & $128.07(5)$ \\
\hline $\mathrm{d}_{\mathrm{Co2}-\mathrm{P} 4}$ & $2.225(1)$ & $\angle \mathrm{N} 4-\mathrm{Co} 2-\mathrm{N} 5$ & $174.2(1)$ \\
\hline $\mathrm{d}_{\mathrm{Co2}-\mathrm{N} 4}$ & $1.944(3)$ & $\angle \mathrm{N} 4-\mathrm{Co} 2-\mathrm{N} 6$ & $92.7(1)$ \\
\hline $\mathrm{d}_{\mathrm{C} 02-\mathrm{N} 5}$ & $1.911(3)$ & $\angle \mathrm{P} 3-\mathrm{Co} 2-\mathrm{N} 6$ & $116.20(9)$ \\
\hline $\mathrm{d}_{\mathrm{Co2} 2 \mathrm{~N} 6}$ & $1.928(3)$ & $\angle \mathrm{P} 4-\mathrm{Co} 2-\mathrm{N} 6$ & $114.35(9)$ \\
\hline $\mathrm{d}_{\mathrm{C} 34-\mathrm{C} 35}$ & $1.447(5)$ & & \\
\hline $\mathrm{d}_{\mathrm{C} 73-\mathrm{C} 74}$ & $1.437(5)$ & & \\
\hline
\end{tabular}


Figure S26. Solid-state structure of (acriPNP)Co(phen) (4-phen). All hydrogen atoms of an acriPNP ligand are omitted for clarity.

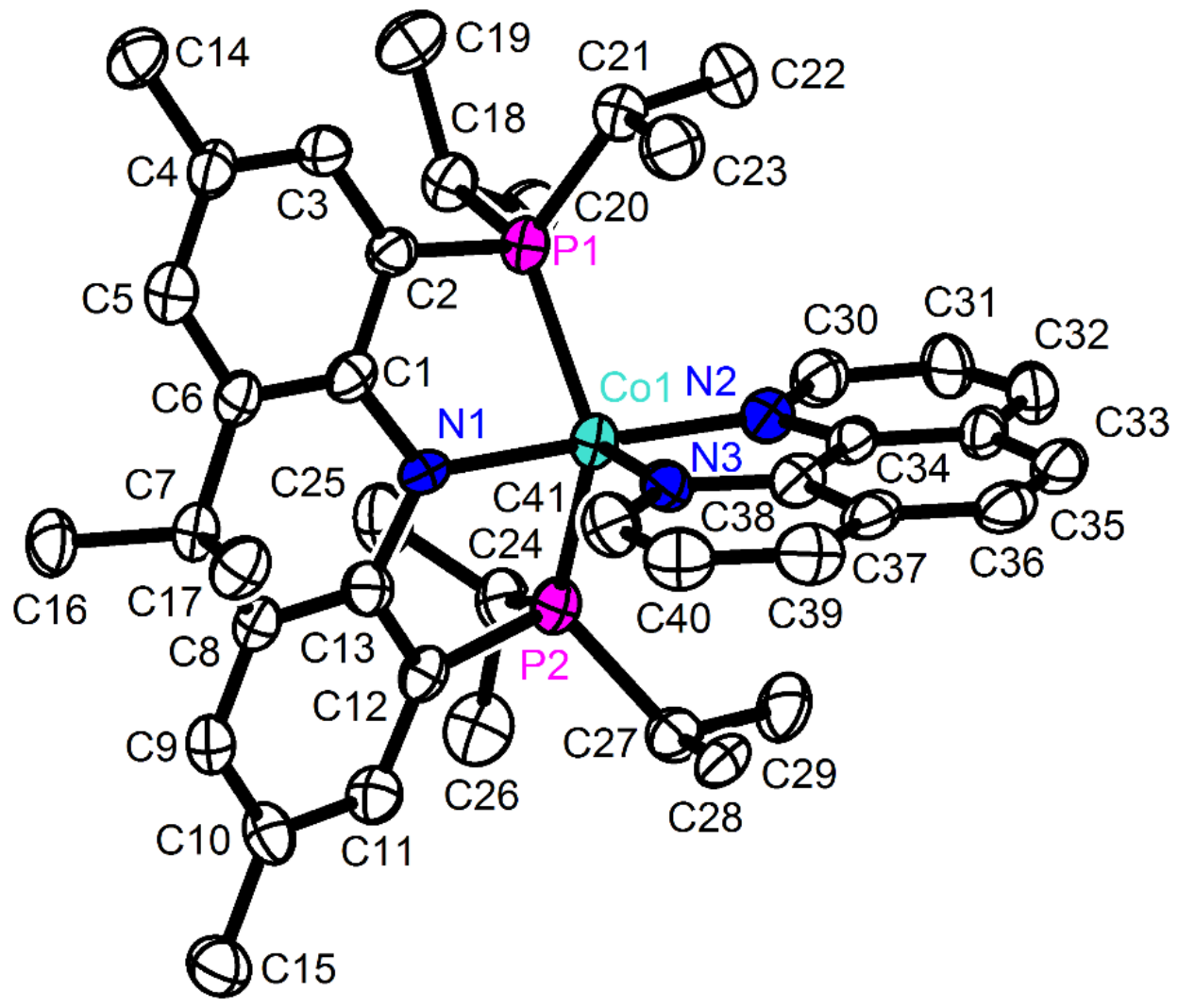

Table S4. Selected bond distances and angles for 4-phen $\left(\AA\right.$ and $\left.^{\circ}\right)$.

\begin{tabular}{cccc}
\hline \multicolumn{2}{c}{ Bond distance } & \multicolumn{2}{c}{ Bond angle } \\
\hline $\mathrm{d}_{\mathrm{Co1} 1 \mathrm{P} 1}$ & $2.221(2)$ & $\angle \mathrm{P} 1-\mathrm{Co} 1-\mathrm{P} 2$ & $128.26(6)$ \\
\hline $\mathrm{d}_{\mathrm{Co1}-\mathrm{P} 2}$ & $2.223(2)$ & $\angle \mathrm{N} 1-\mathrm{Co} 1-\mathrm{N} 2$ & $174.0(2)$ \\
\hline $\mathrm{d}_{\mathrm{Co} 1-\mathrm{N} 1}$ & $1.947(4)$ & $\angle \mathrm{N} 1-\mathrm{Co} 1-\mathrm{N} 3$ & $82.4(2)$ \\
\hline $\mathrm{d}_{\mathrm{Co1}-\mathrm{N} 2}$ & $1.913(4)$ & & \\
\hline $\mathrm{d}_{\mathrm{Co} 1-\mathrm{N} 3}$ & $1.935(4)$ & & \\
\hline $\mathrm{d}_{\mathrm{C} 34-\mathrm{C} 38}$ & $1.399(7)$ & & \\
\hline $\mathrm{d}_{\mathrm{N} 1-\mathrm{C} 1}$ & $1.376(6)$ & & \\
\hline $\mathrm{d}_{\mathrm{N} 1-\mathrm{C} 13}$ & $1.384(6)$ & & \\
\hline
\end{tabular}


Figure S27. Solid-state structure of $\left\{\left({ }^{a c r i P N P}\right) \operatorname{Co}(\mathrm{bpy})\right\}\left\{\mathrm{NTf}_{2}\right\}$ (5-bpy). All hydrogen atoms of an acriPNP ligand are omitted for clarity.

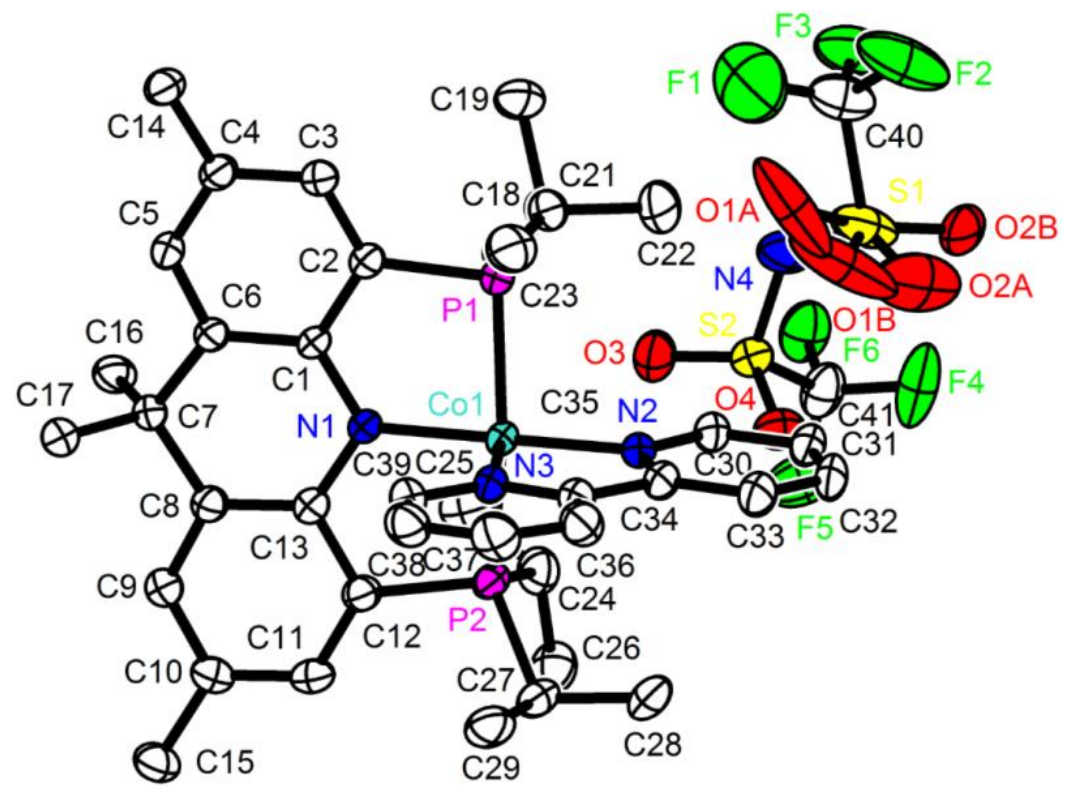

Table S5. Selected bond distances and angles for 5-bpy ( $\left(\right.$ and $\left.^{\circ}\right)$.

\begin{tabular}{cccc}
\hline \multicolumn{2}{c}{ Bond distance } & \multicolumn{2}{c}{ Bond angle } \\
\hline $\mathrm{d}_{\mathrm{C} 1-\mathrm{P} 1}$ & $2.2408(9)$ & $\angle \mathrm{P} 1-\mathrm{Co} 1-\mathrm{P} 2$ & $159.02(4)$ \\
\hline $\mathrm{d}_{\mathrm{C} 01-\mathrm{P} 2}$ & $2.2265(9)$ & $\angle \mathrm{N} 1-\mathrm{Co} 1-\mathrm{N} 2$ & $179.0(1)$ \\
\hline $\mathrm{d}_{\mathrm{C} 01-\mathrm{N} 1}$ & $1.948(3)$ & $\angle \mathrm{N} 1-\mathrm{Co} 1-\mathrm{N} 3$ & $80.8(1)$ \\
\hline $\mathrm{d}_{\mathrm{Co1} 1 \mathrm{~N} 2}$ & $1.933(3)$ & & \\
\hline $\mathrm{d}_{\mathrm{Co} 1-\mathrm{N} 3}$ & $2.029(3)$ & & \\
\hline $\mathrm{d}_{\mathrm{C} 34-\mathrm{C} 35}$ & $1.471(5)$ & & \\
\hline $\mathrm{d}_{\mathrm{N} 1-\mathrm{C} 1}$ & $1.392(4)$ & & \\
\hline $\mathrm{d}_{\mathrm{N} 1-\mathrm{C} 13}$ & $1.392(4)$ & & \\
\hline
\end{tabular}


Figure S28. Solid-state structure of $\left\{\left({ }^{\text {acripNP }}\right) \mathrm{Co}(\right.$ phen $\left.)\right\}\left\{\mathrm{NTf}_{2}\right\}$ (5-phen). All hydrogen atoms of an acripNP ligand are omitted for clarity.
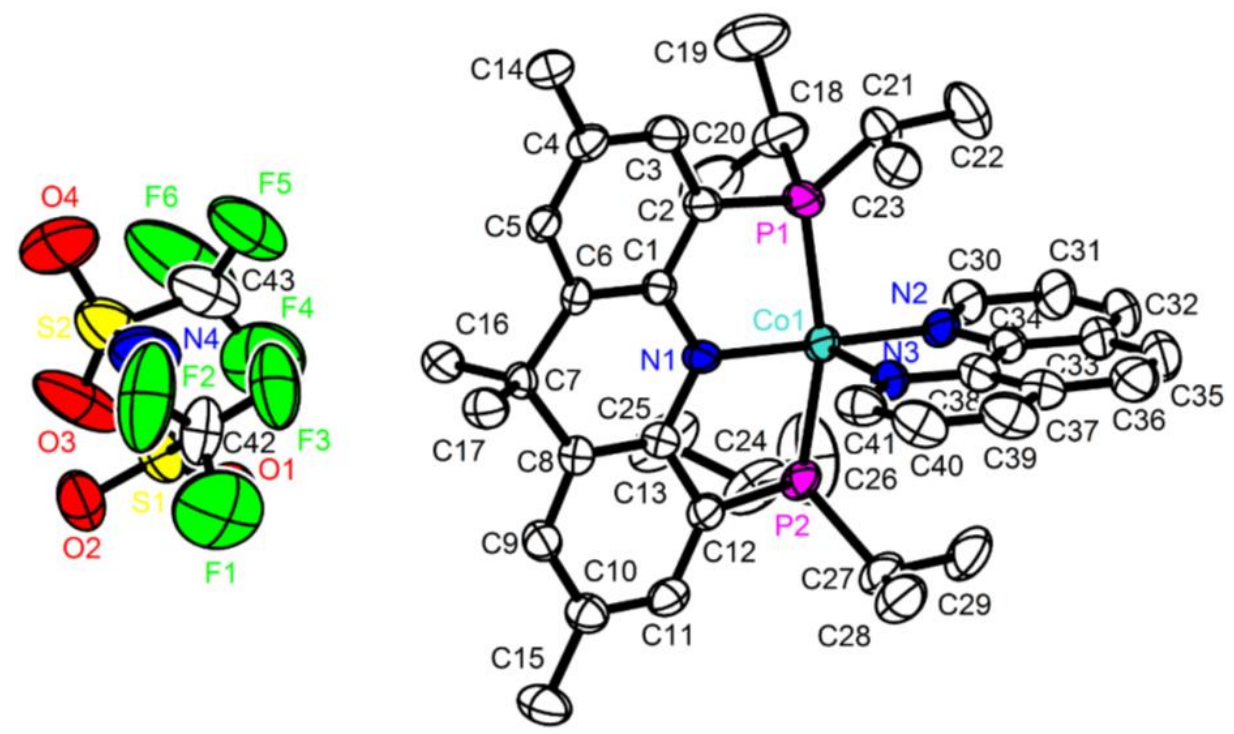

Table S6. Selected bond distances and angles for 5-phen $\left(\AA\right.$ and $\left.{ }^{\circ}\right)$.

\begin{tabular}{cccc}
\hline \multicolumn{2}{c}{ Bond distance } & \multicolumn{2}{c}{ Bond angle } \\
\hline $\mathrm{d}_{\mathrm{Co1} 1 \mathrm{P} 1}$ & $2.236(2)$ & $\angle \mathrm{P} 1-\mathrm{Co} 1-\mathrm{P} 2$ & $161.45(8)$ \\
\hline $\mathrm{d}_{\mathrm{Co1} 1 \mathrm{P} 2}$ & $2.243(2)$ & $\angle \mathrm{N} 1-\mathrm{Co} 1-\mathrm{N} 2$ & $178.4(2)$ \\
\hline $\mathrm{d}_{\mathrm{Co1}-\mathrm{N} 1}$ & $1.940(4)$ & $\angle \mathrm{N} 1-\mathrm{Co} 1-\mathrm{N} 3$ & $99.1(2)$ \\
\hline $\mathrm{d}_{\mathrm{Co1} 1 \mathrm{~N} 2}$ & $1.942(5)$ & & \\
\hline $\mathrm{d}_{\mathrm{Co1}-\mathrm{N} 3}$ & $2.042(5)$ & & \\
\hline $\mathrm{d}_{\mathrm{C} 34-\mathrm{C} 38}$ & $1.433(8)$ & & \\
\hline $\mathrm{d}_{\mathrm{N} 1-\mathrm{C} 1}$ & $1.400(7)$ & & \\
\hline $\mathrm{d}_{\mathrm{N} 1-\mathrm{C} 13}$ & $1.406(7)$ & & \\
\hline
\end{tabular}


Figure S29. Solid-state structure of ( $\left.{ }^{a c r i P N P}\right) \mathrm{Co}(\mathrm{biph})$ (6). All hydrogen atoms of an ${ }^{\text {acripNP }}$ ligand are omitted for clarity.

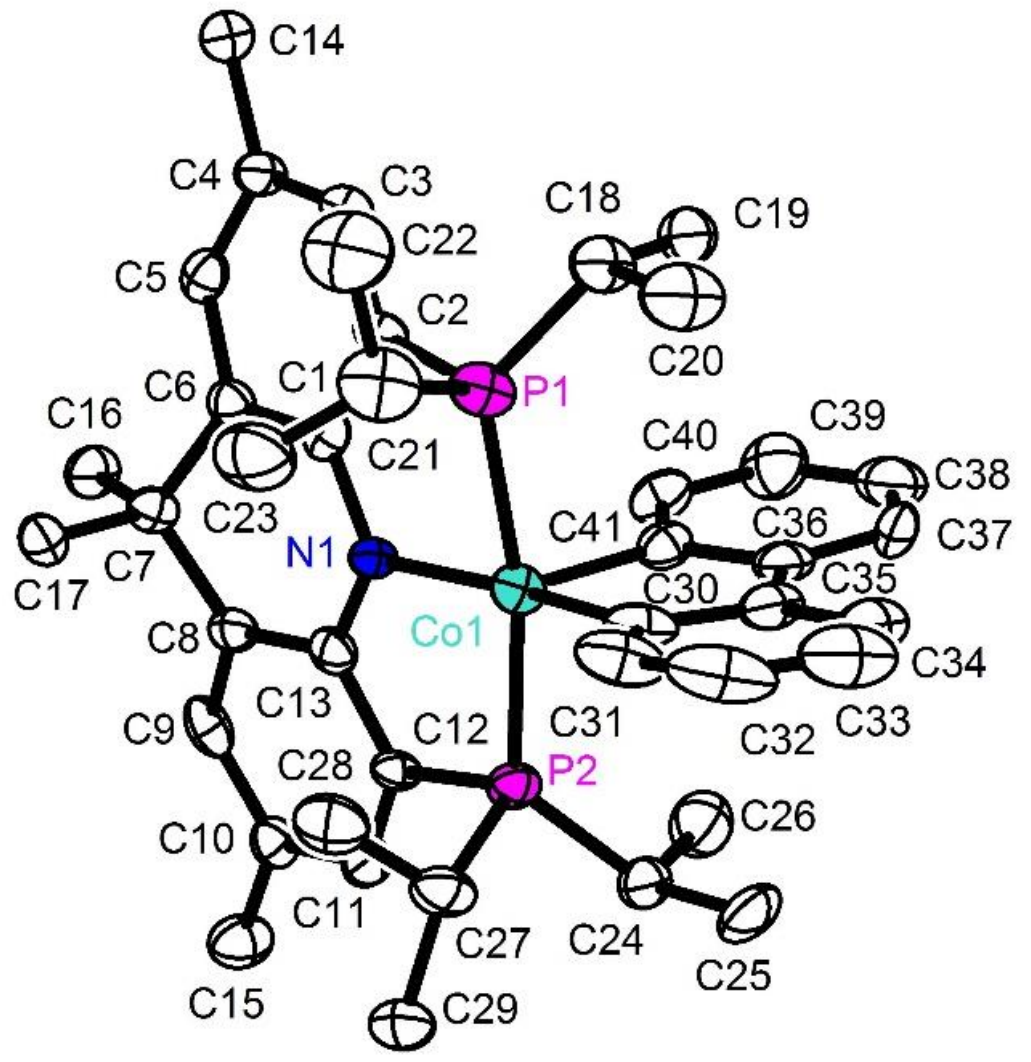

Table S7. Selected bond distances and angles for $\mathbf{6}\left(\AA\right.$ and $\left.^{\circ}\right)$.

\begin{tabular}{|c|c|c|c|}
\hline \multicolumn{2}{|c|}{ Bond distance } & \multicolumn{2}{|c|}{ Bond angle } \\
\hline $\mathrm{d}_{\mathrm{Co1-P} 1}$ & $2.240(2)$ & $\angle \mathrm{P} 1-\mathrm{Co} 1-\mathrm{P} 2$ & $168.89(7)$ \\
\hline $\mathrm{d}_{\mathrm{Co} 1-\mathrm{P} 2}$ & $2.203(2)$ & $\angle \mathrm{N} 1-\mathrm{Co} 1-\mathrm{C} 30$ & $170.4(2)$ \\
\hline $\mathrm{d}_{\mathrm{Co} 1-\mathrm{N} 1}$ & $1.947(4)$ & $\angle \mathrm{N} 1-\mathrm{Co} 1-\mathrm{C} 41$ & $105.7(2)$ \\
\hline d Co1-C30 & $1.924(6)$ & & \\
\hline $\mathrm{d}_{\mathrm{Co1-C} 41}$ & $1.892(6)$ & & \\
\hline $\mathrm{d}_{\mathrm{N} 1-\mathrm{C} 1}$ & $1.415(7)$ & & \\
\hline $\mathrm{d}_{\mathrm{N} 1-\mathrm{C} 13}$ & $1.400(6)$ & & \\
\hline
\end{tabular}


Figure S30. Solid-state structure of $\left\{\left({ }^{\text {acripNP}}\right) \operatorname{Co}(\mathrm{ppy})\right\}\left\{\mathrm{NTf}_{2}\right\}$ (7-ppy). All hydrogen atoms of an acriPNP ligand are omitted for clarity.

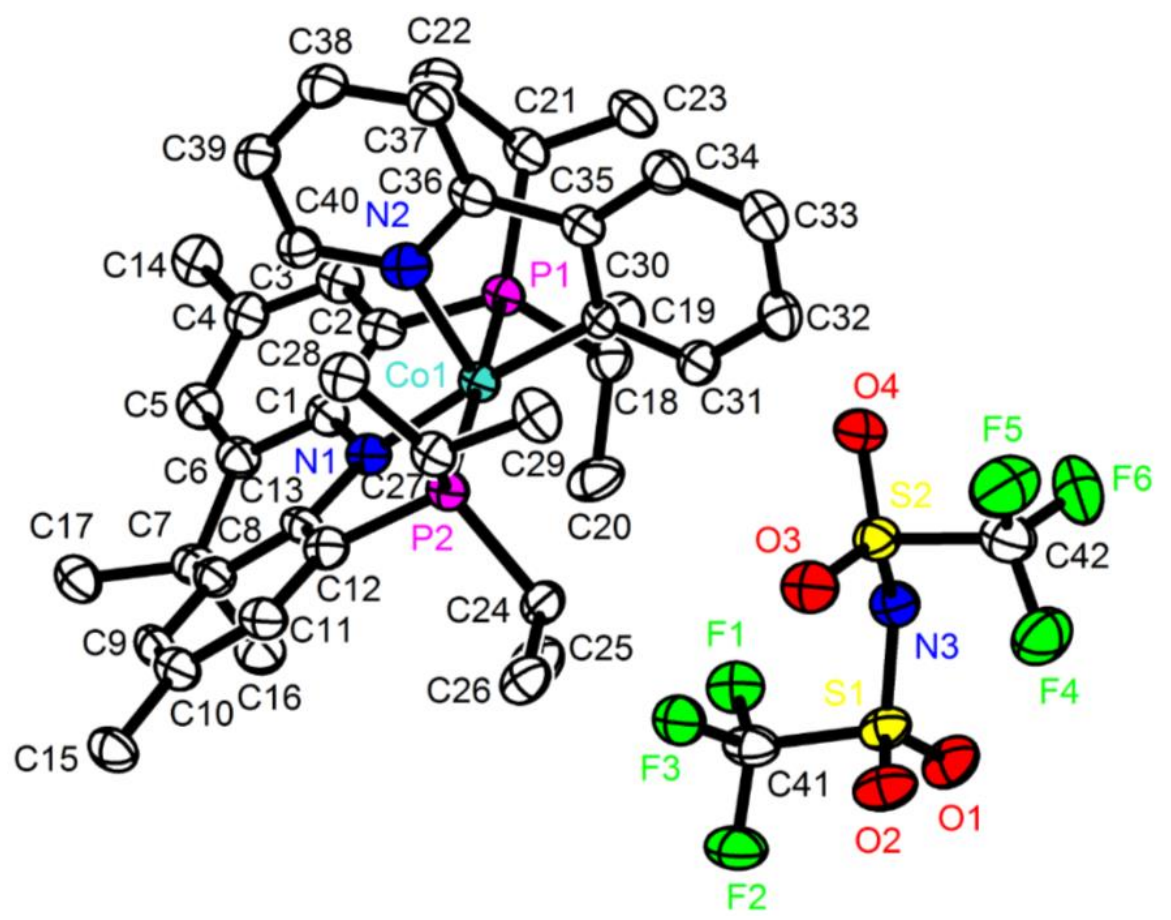

Table S8. Selected bond distances and angles for 7-ppy $\left(\AA\right.$ and $\left.^{\circ}\right)$.

\begin{tabular}{cccc}
\hline \multicolumn{2}{c}{ Bond distance } & \multicolumn{2}{c}{ Bond angle } \\
\hline $\mathrm{d}_{\mathrm{C} 1-\mathrm{P} 1}$ & $2.235(1)$ & $\angle \mathrm{P} 1-\mathrm{Co} 1-\mathrm{P} 2$ & $170.70(5)$ \\
\hline $\mathrm{d}_{\mathrm{Co1} 1 \mathrm{P} 2}$ & $2.225(1)$ & $\angle \mathrm{N} 1-\mathrm{Co} 1-\mathrm{N} 2$ & $101.6(2)$ \\
\hline $\mathrm{d}_{\mathrm{Co1} 1 \mathrm{~N} 1}$ & $1.892(3)$ & $\angle \mathrm{N} 1-\mathrm{Co} 1-\mathrm{C} 30$ & $174.6(1)$ \\
\hline $\mathrm{d}_{\mathrm{Co1} 1 \mathrm{~N} 2}$ & $1.891(4)$ & & \\
\hline $\mathrm{d}_{\mathrm{Co1} 1 \mathrm{C} 30}$ & $1.930(4)$ & & \\
\hline $\mathrm{d}_{\mathrm{N} 1-\mathrm{C} 1}$ & $1.416(6)$ & & \\
\hline $\mathrm{d}_{\mathrm{N} 1-\mathrm{C} 13}$ & $1.417(5)$ & & \\
\hline
\end{tabular}


Figure S31. Solid-state structure of $\left\{\left({ }^{\text {acripNP}}\right) \operatorname{Co}(\mathrm{bzq})\right\}\left\{\mathrm{NTf}_{2}\right\}$ (7-bzq). All hydrogen atoms of an acripNP ligand are omitted for clarity.

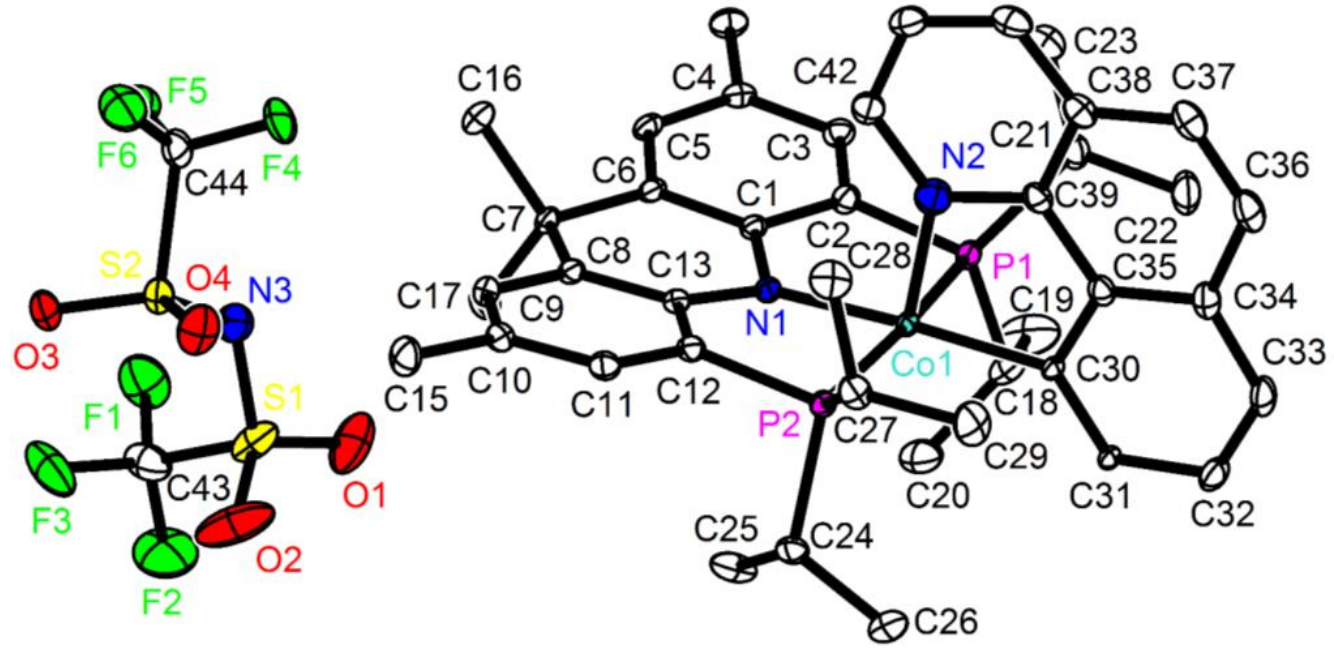

Table S9. Selected bond distances and angles for 7-bzq $\left(\AA\right.$ and $\left.^{\circ}\right)$.

\begin{tabular}{cccc}
\hline \multicolumn{2}{c}{ Bond distance } & \multicolumn{2}{c}{ Bond angle } \\
\hline $\mathrm{d}_{\mathrm{C} 1-\mathrm{P} 1}$ & $2.2235(5)$ & $\angle \mathrm{P} 1-\mathrm{Co} 1-\mathrm{P} 2$ & $170.14(2)$ \\
\hline $\mathrm{d}_{\mathrm{Co1} 1 \mathrm{P} 2}$ & $2.2298(5)$ & $\angle \mathrm{N} 1-\mathrm{Co} 1-\mathrm{N} 2$ & $98.41(7)$ \\
\hline $\mathrm{d}_{\mathrm{Co1} 1 \mathrm{~N} 1}$ & $1.889(2)$ & $\angle \mathrm{N} 1-\mathrm{Co} 1-\mathrm{C} 30$ & $175.49(7)$ \\
\hline $\mathrm{d}_{\mathrm{C} 1-\mathrm{N} 2}$ & $1.898(2)$ & & \\
\hline $\mathrm{d}_{\mathrm{Co1} 1-\mathrm{C} 30}$ & $1.959(2)$ & & \\
\hline $\mathrm{d}_{\mathrm{N} 1-\mathrm{C} 1}$ & $1.407(2)$ & & \\
\hline $\mathrm{d}_{\mathrm{N} 1-\mathrm{C} 13}$ & $1.413(2)$ & & \\
\hline
\end{tabular}


Figure S32. Solid-state structure of $\{($ acripNP)Co(biph $)\}\{\mathrm{K}(18-\mathrm{C}-6)\}(8)$. All hydrogen atoms of an acripNP ligand are omitted for clarity.

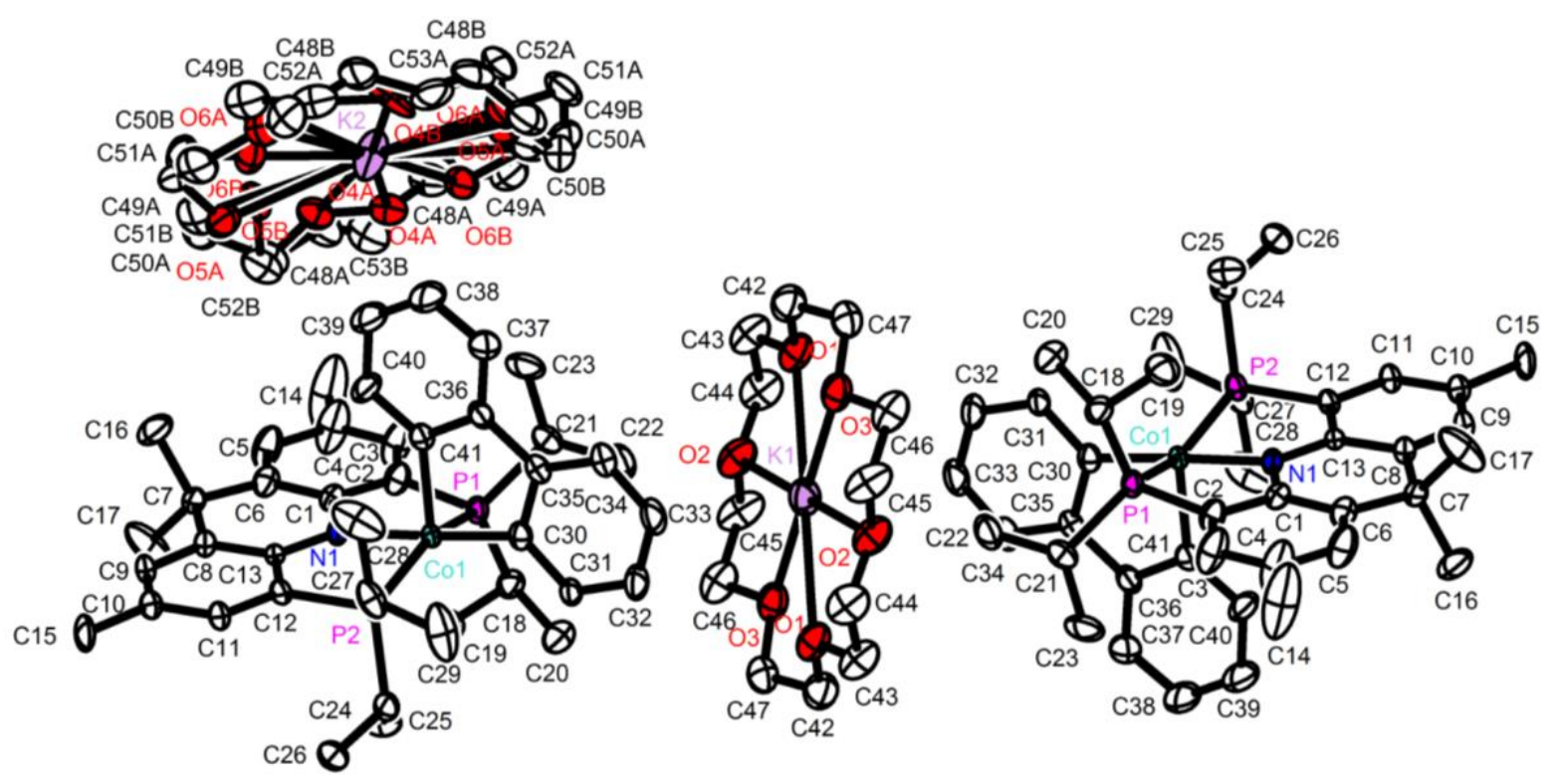

Table S10. Selected bond distances and angles for $\mathbf{8}\left(\AA \AA\right.$ and $\left.^{\circ}\right)$.

\begin{tabular}{cccc}
\hline \multicolumn{2}{c}{ Bond distance } & \multicolumn{2}{c}{ Bond angle } \\
\hline $\mathrm{d}_{\mathrm{Co1} 1 \mathrm{P} 1}$ & $2.194(1)$ & $\angle \mathrm{P} 1-\mathrm{Co} 1-\mathrm{P} 2$ & $152.14(8)$ \\
\hline $\mathrm{d}_{\mathrm{Co1}-\mathrm{P} 2}$ & $2.196(1)$ & $\angle \mathrm{N} 1-\mathrm{Co} 1-\mathrm{C} 30$ & $177.5(3)$ \\
\hline $\mathrm{d}_{\mathrm{Co1} 1 \mathrm{~N} 1}$ & $1.995(3)$ & $\angle \mathrm{N} 1-\mathrm{Co} 1-\mathrm{C} 41$ & $93.8(3)$ \\
\hline $\mathrm{d}_{\mathrm{Co1} 1-\mathrm{C} 30}$ & $1.926(4)$ & & \\
\hline $\mathrm{d}_{\mathrm{Co1} 1-\mathrm{C} 41}$ & $1.964(4)$ & & \\
\hline $\mathrm{d}_{\mathrm{N} 1-\mathrm{C} 1}$ & $1.382(5)$ & & \\
\hline $\mathrm{d}_{\mathrm{N} 1-\mathrm{C} 13}$ & $1.375(5)$ & & \\
\hline
\end{tabular}


Figure S33. Core structures of (a) 4-bpy, (b) 5-bpy, (c) 4-phen and (d) 5-phen. The distances of a cobalt atom from the red plane defined by two phosphorus and one nitrogen are shown.

(a)

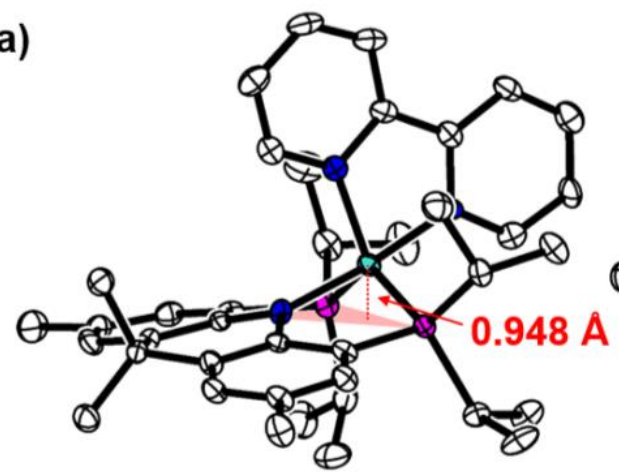

(c)

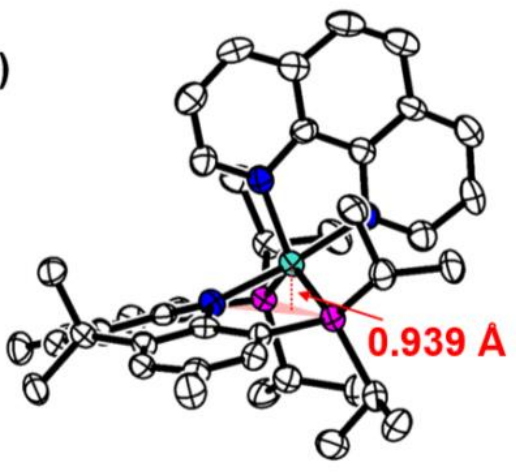

(b)

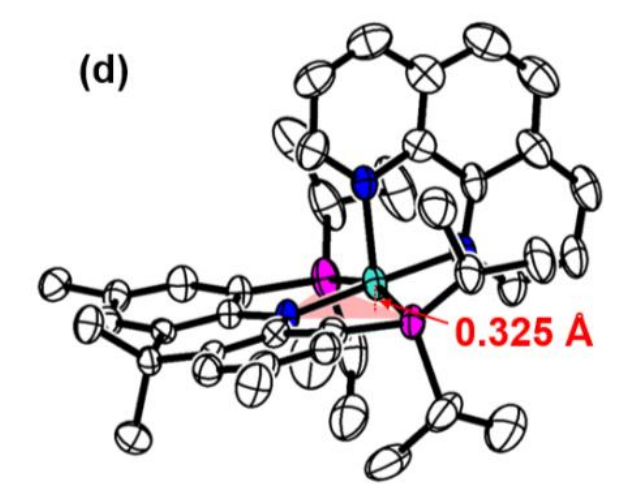

Figure S34. Core structures of (a) 4-bpy, (b) 5-bpy, (c) 4-phen and (d) 5-phen. The distances of a nitrogen atom from the red plane defined by two carbon and one cobalt are shown.

(a)

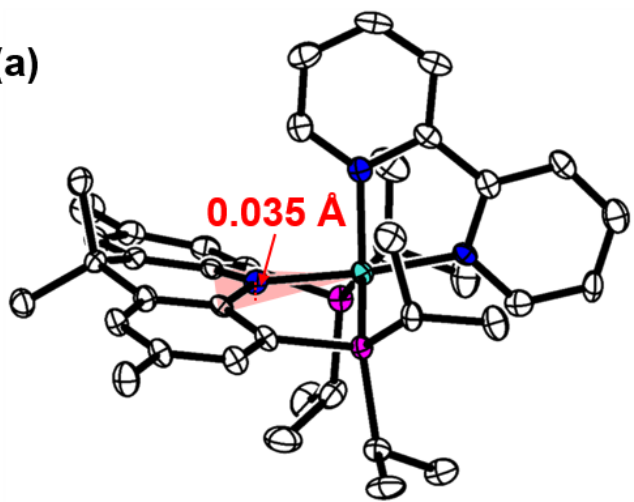

(c)

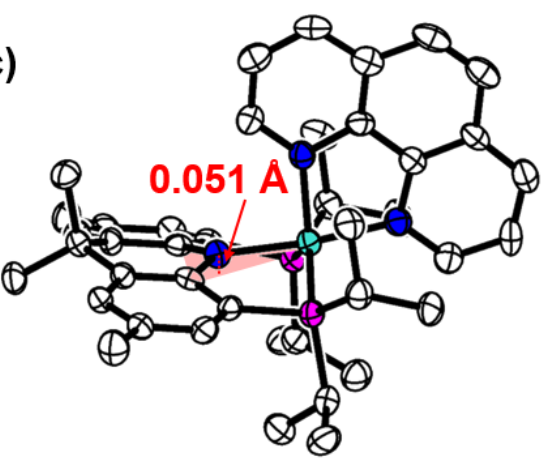

(b)

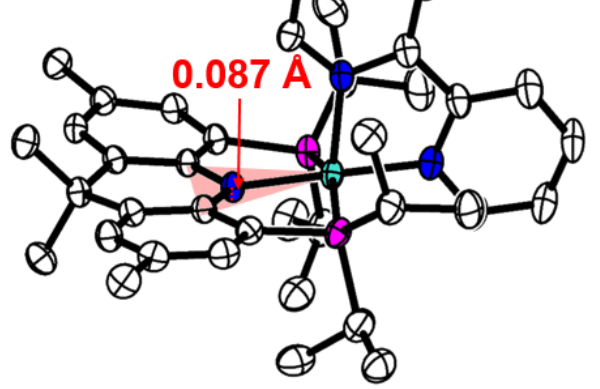

(d)

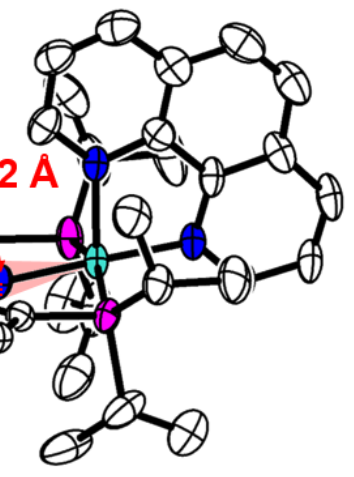




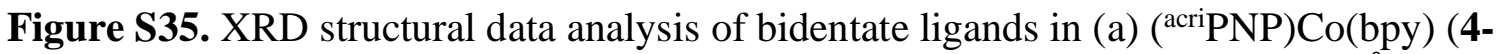
bpy) and (b) (acriPNP)Co(phen) (4-phen) based on the XRD data; bond length in $\AA$.

(a)

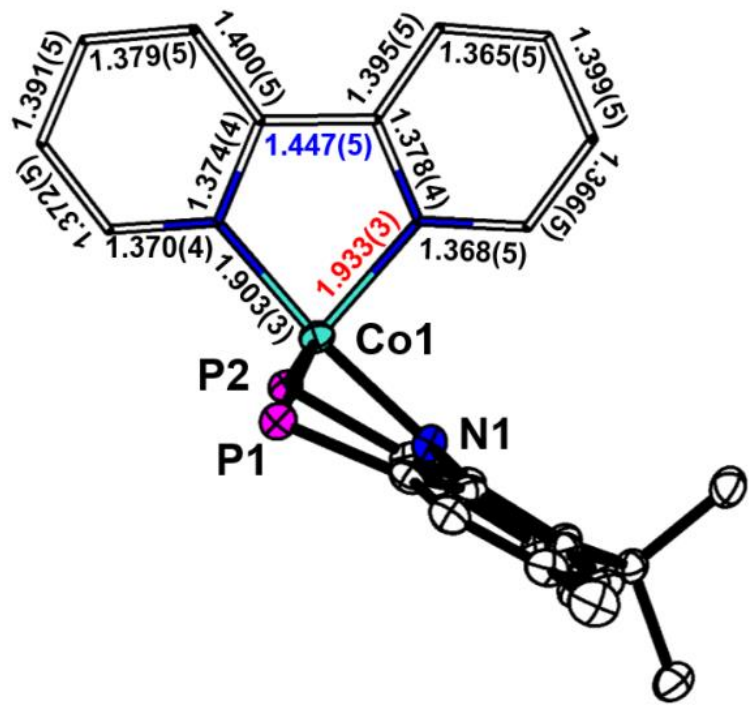

(b)

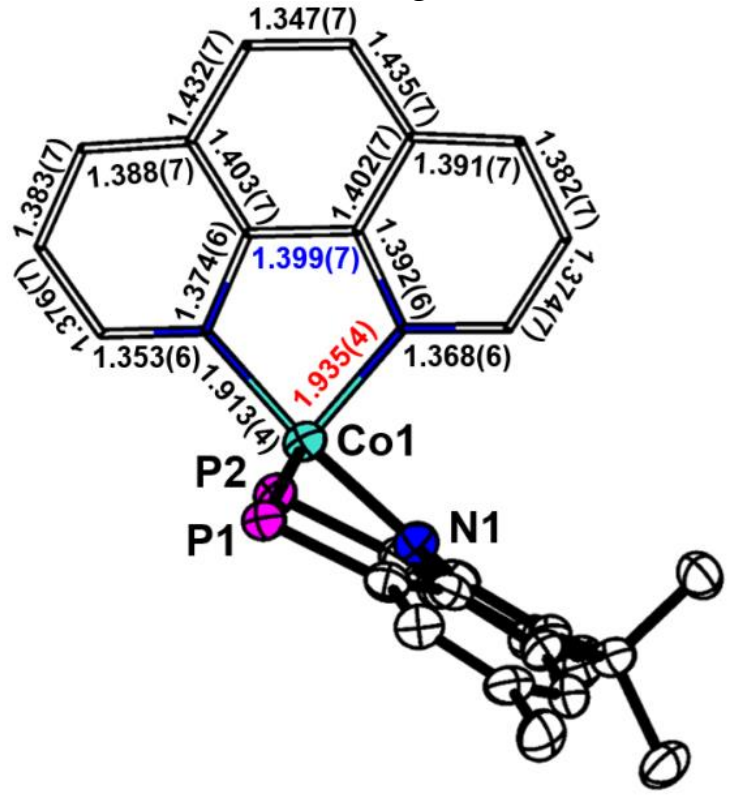

Figure S36. UV-Vis spectra of (acriPNP)Co(ppy) (2-ppy, black) and (acriPNP)Co(bzq) (2-bzq, red) in THF at room temperature.

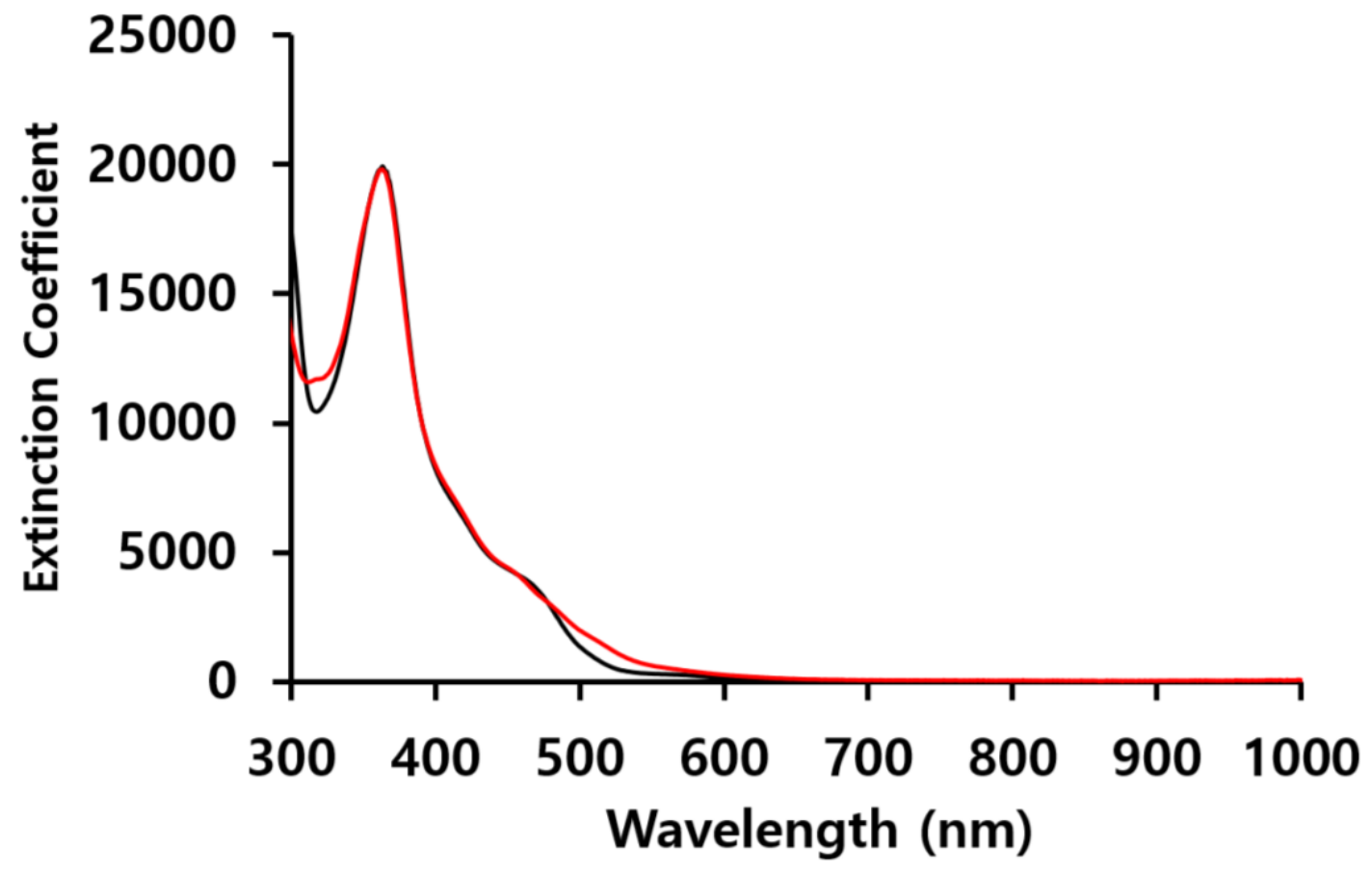




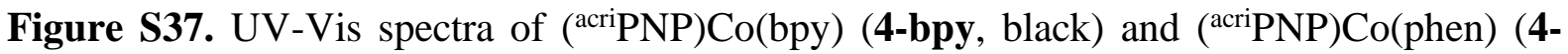
phen, red) in THF at room temperature.

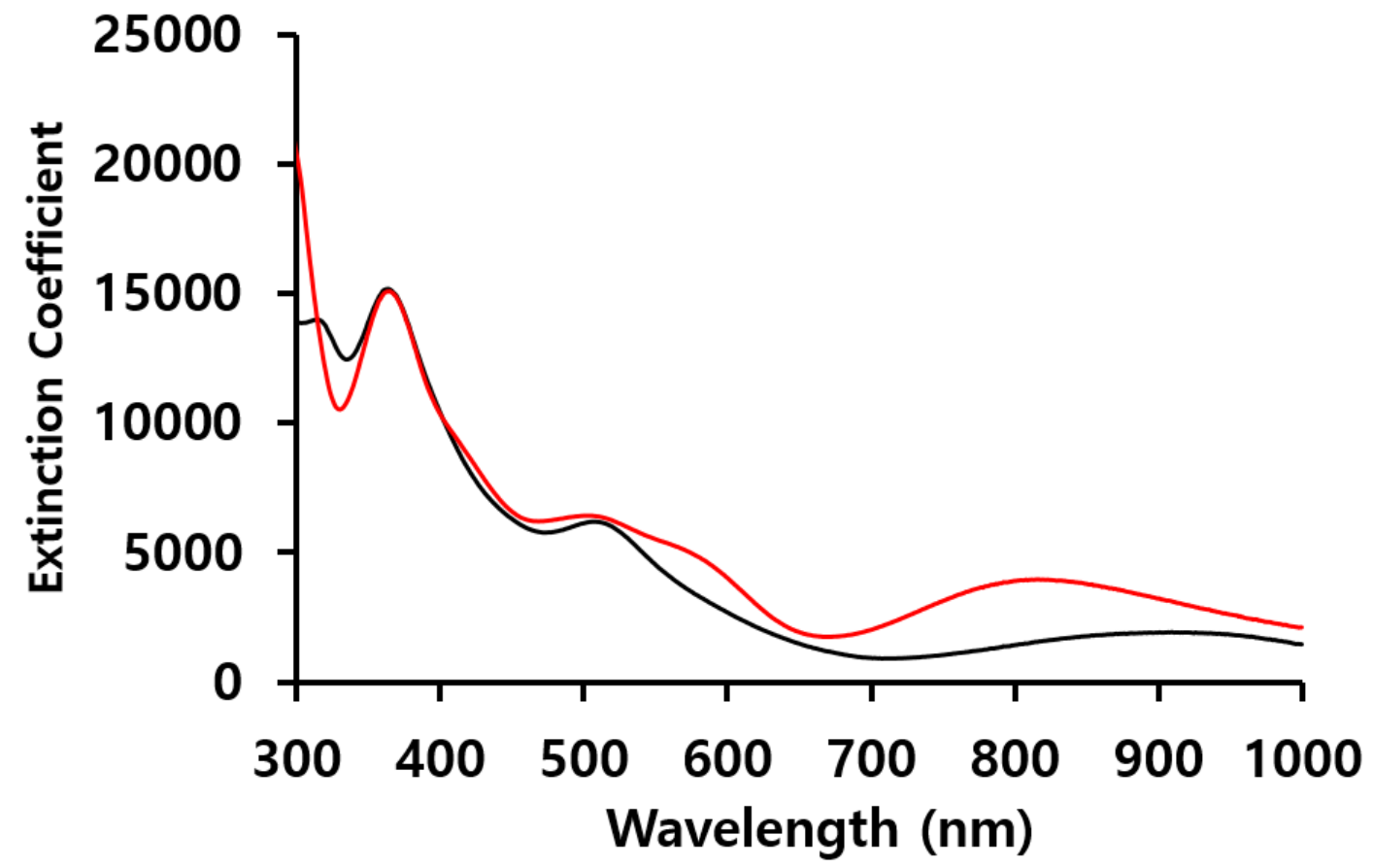

Figure S38. UV-Vis spectra of $\{($ acriPNP)Co(bpy $)\}\left\{\mathrm{NTf}_{2}\right\}$ (5-bpy, black) and $\left\{\left({ }^{\text {acripNP}}\right) \mathrm{Co}(\right.$ phen $\left.)\right\}\left\{\mathrm{NTf}_{2}\right\}$ (5-phen, red) in THF at room temperature.

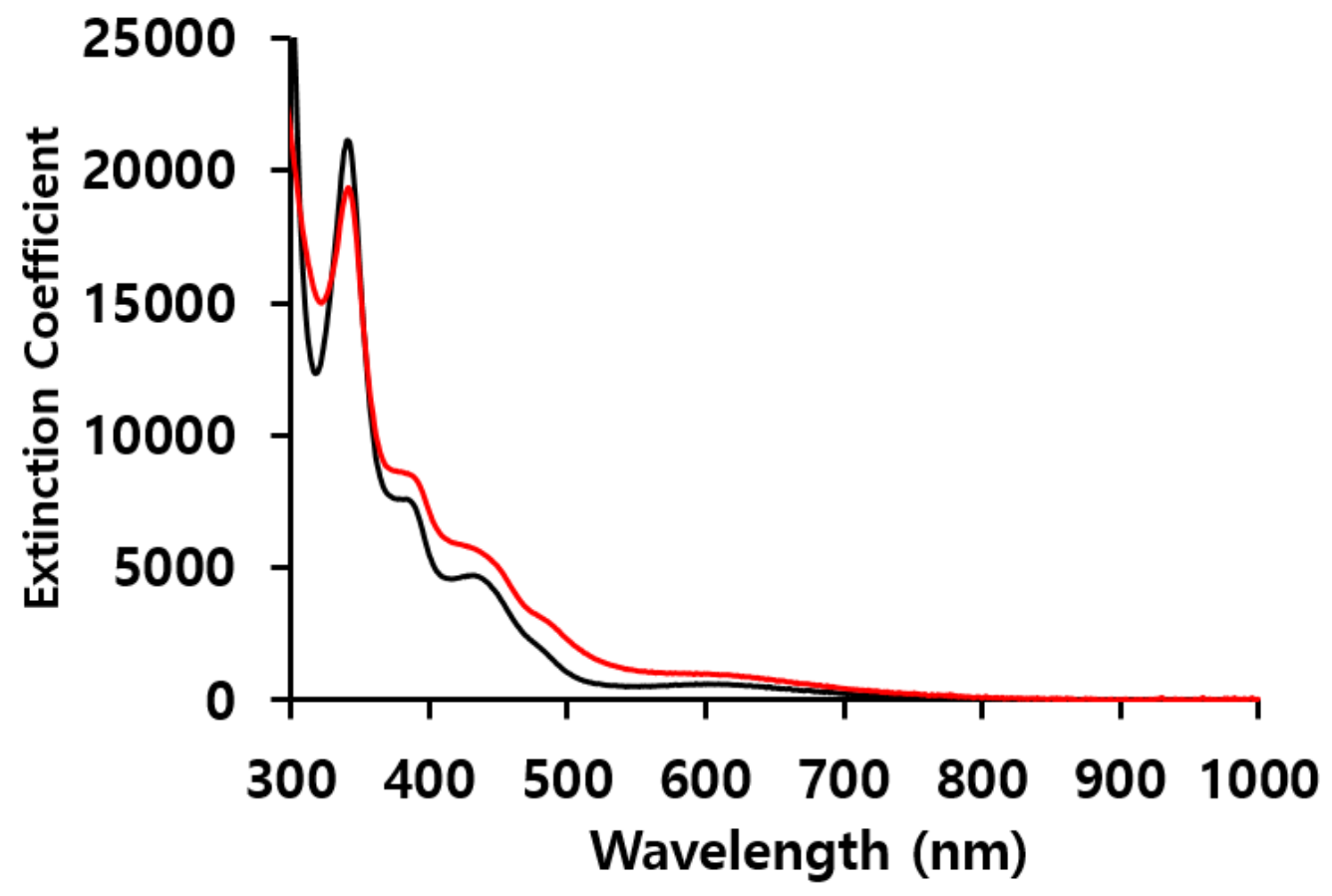


Figure S39. UV-Vis spectra of (acriPNP)Co(biph) (6) in THF at room temperature.

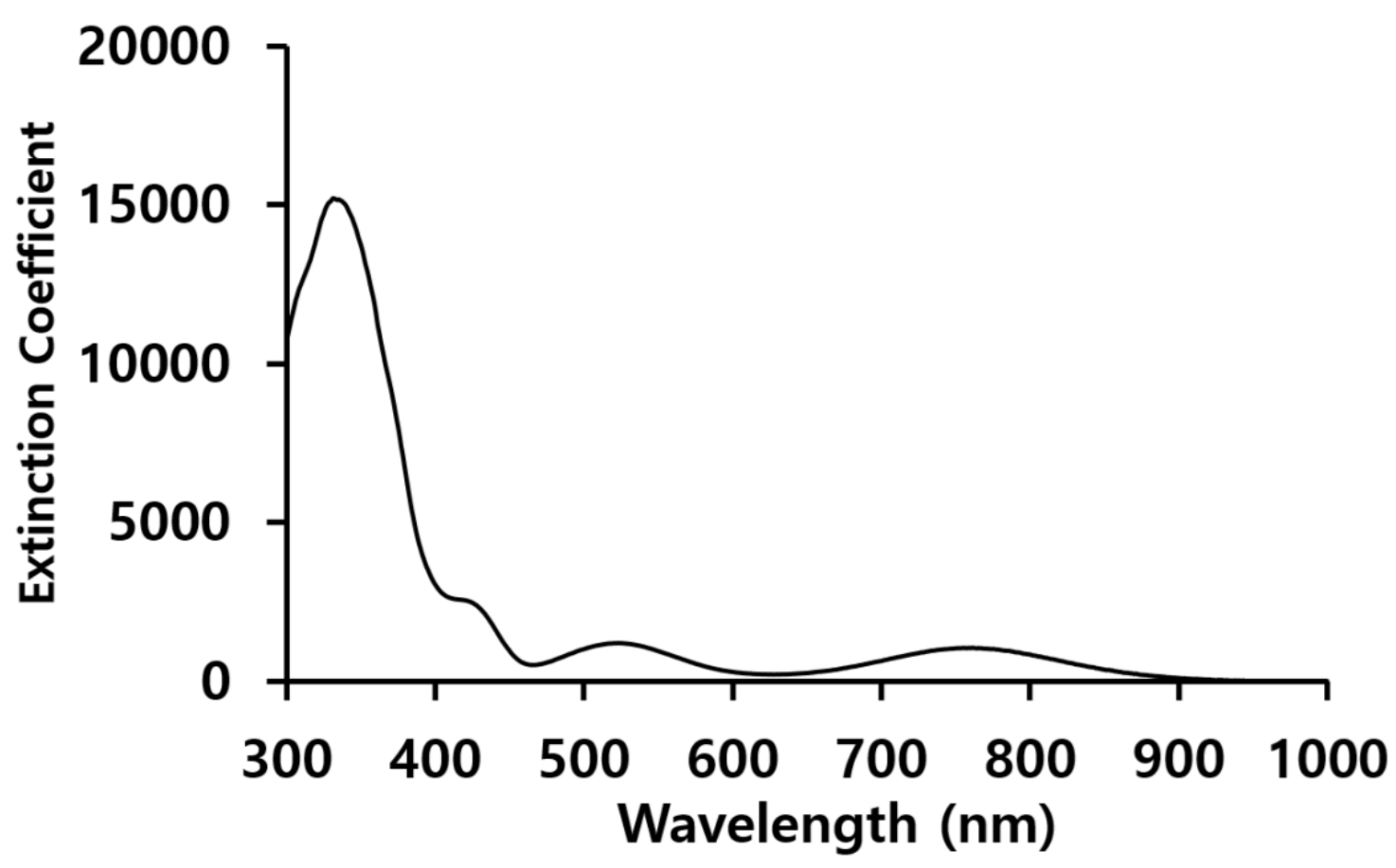

Figure S40. UV-Vis spectra of $\quad\left\{(\right.$ acripNP)Co(ppy) $\}\left\{\mathrm{NTf}_{2}\right\} \quad$ (7-ppy, black) and $\left\{\left({ }^{\text {acripNP }}\right) \mathrm{Co}(\mathrm{bzq})\right\}\left\{\mathrm{NTf}_{2}\right\}(\mathbf{7 - b z q}$, red $)$ in THF at room temperature.

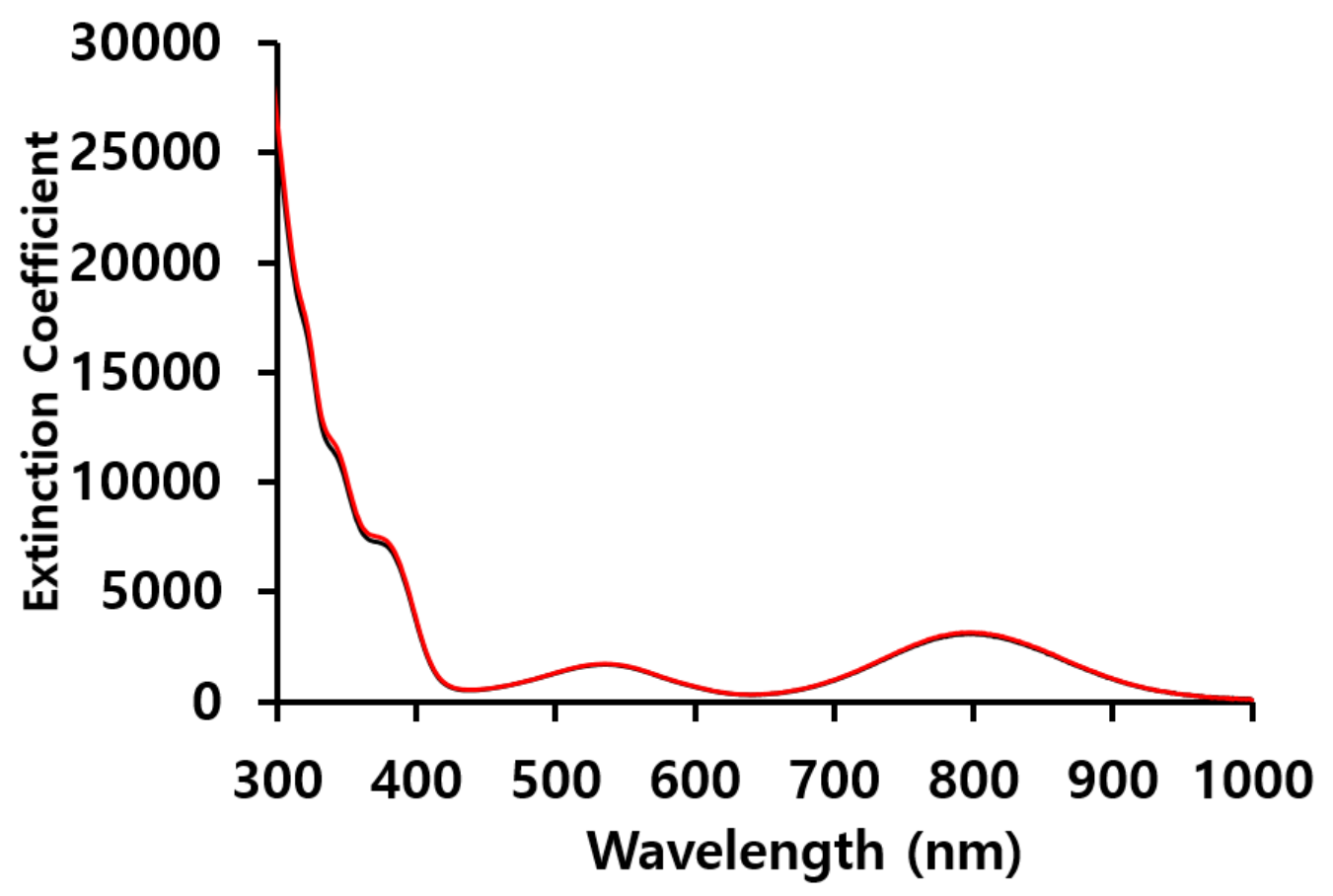


Figure S41. UV-Vis spectrum of $\left\{\left({ }^{\text {acripNP }}\right) \operatorname{Co}(\right.$ biph $\left.)\right\}\{\mathrm{K}(18-\mathrm{C}-6)\}$ (8) in THF at room temperature.

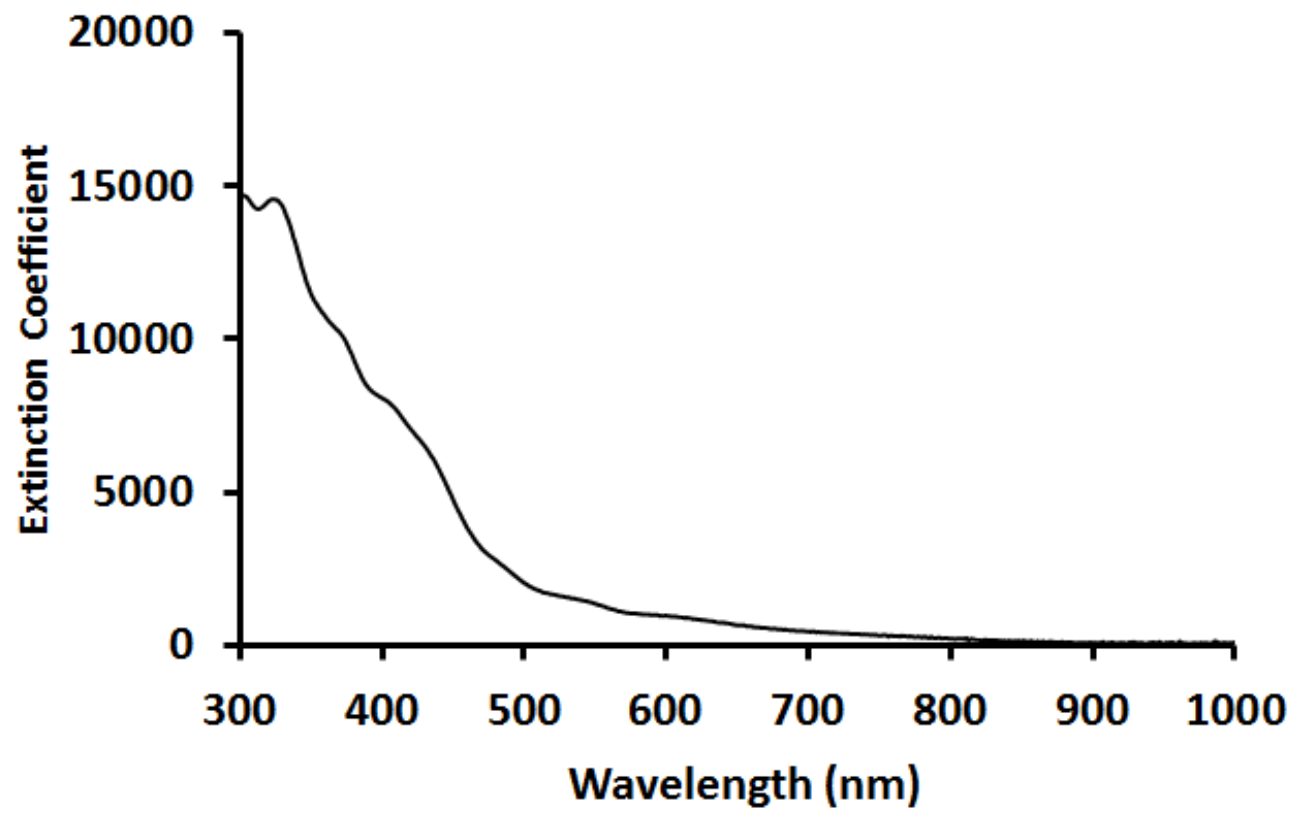

Figure S42. X-Band EPR spectrum of (acriPNP)Co(ppy) (2-ppy) in toluene (black) at $10 \mathrm{~K}$ with a simulated spectrum (red).

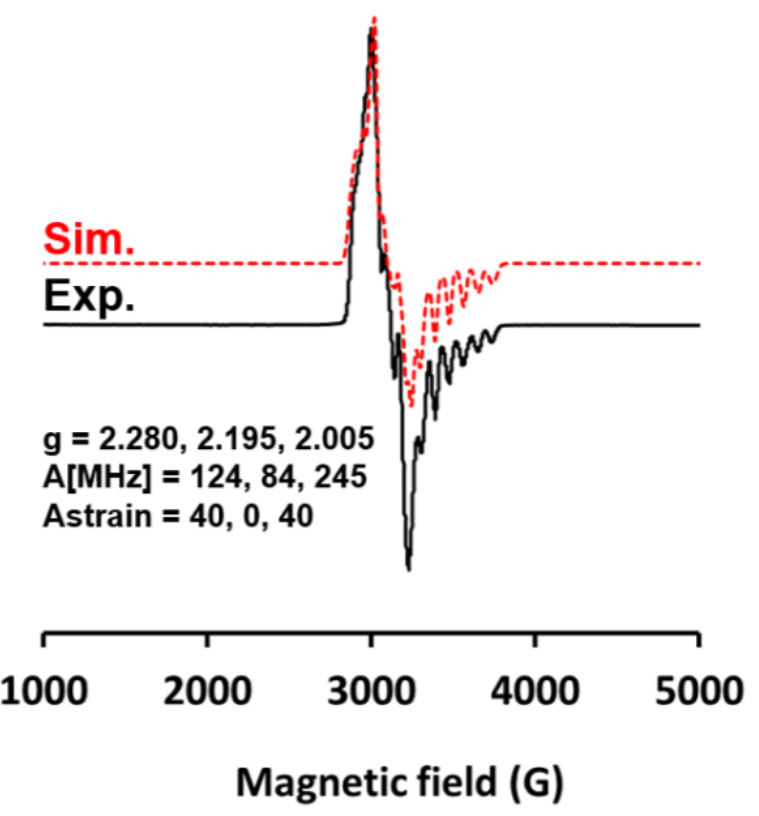


Figure S43. X-Band EPR spectrum of (acriPNP)Co(bzq) (2-bzq) in toluene (black) at $10 \mathrm{~K}$ with a simulated spectrum (red).

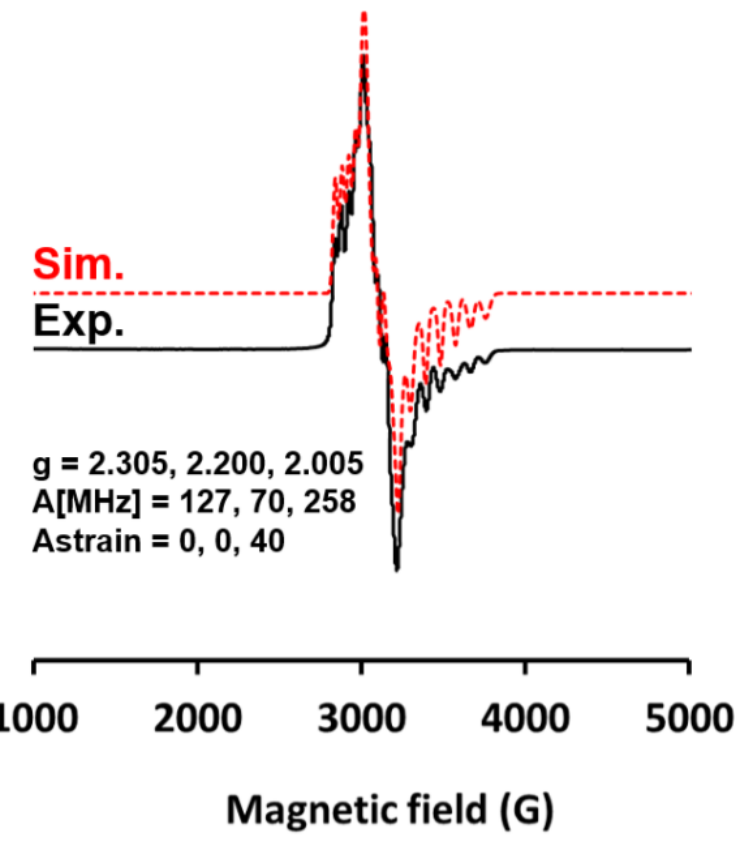

Figure S44. X-Band EPR spectrum of $\left\{(\right.$ acriPNP)Co(bpy) $\}\left\{\mathrm{NTf}_{2}\right\}$ (5-bpy) in toluene (black) at $10 \mathrm{~K}$ with a simulated spectrum (red).

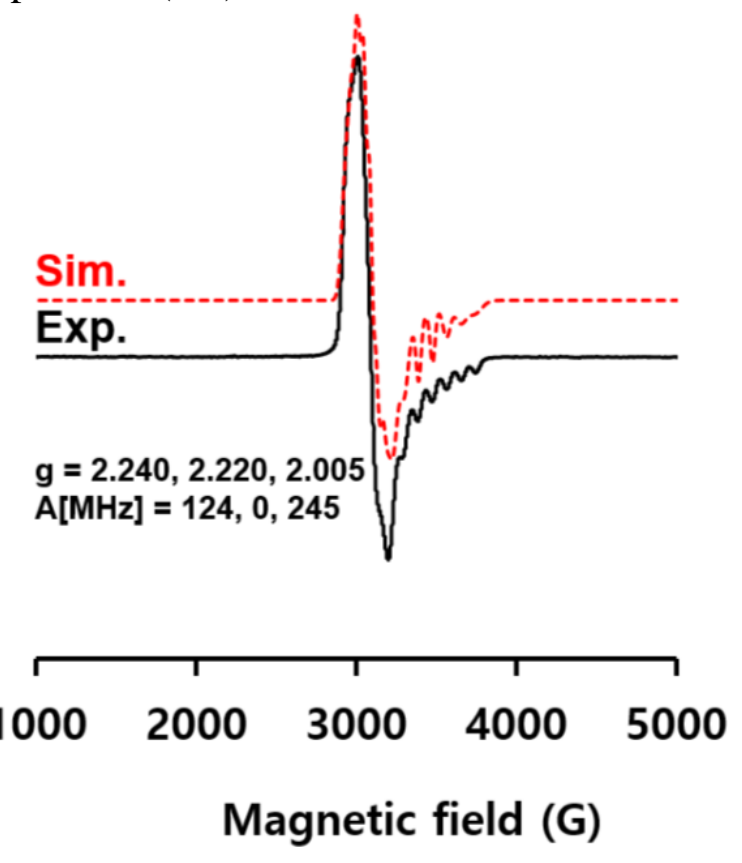


Figure S45. X-Band EPR spectrum of $\left\{\right.$ ( $\left.\left.{ }^{\text {acriPNP}}\right) \mathrm{Co}(\mathrm{phen})\right\}\left\{\mathrm{NTf}_{2}\right\}$ (5-phen) in toluene (black) at $10 \mathrm{~K}$ with a simulated spectrum (red).

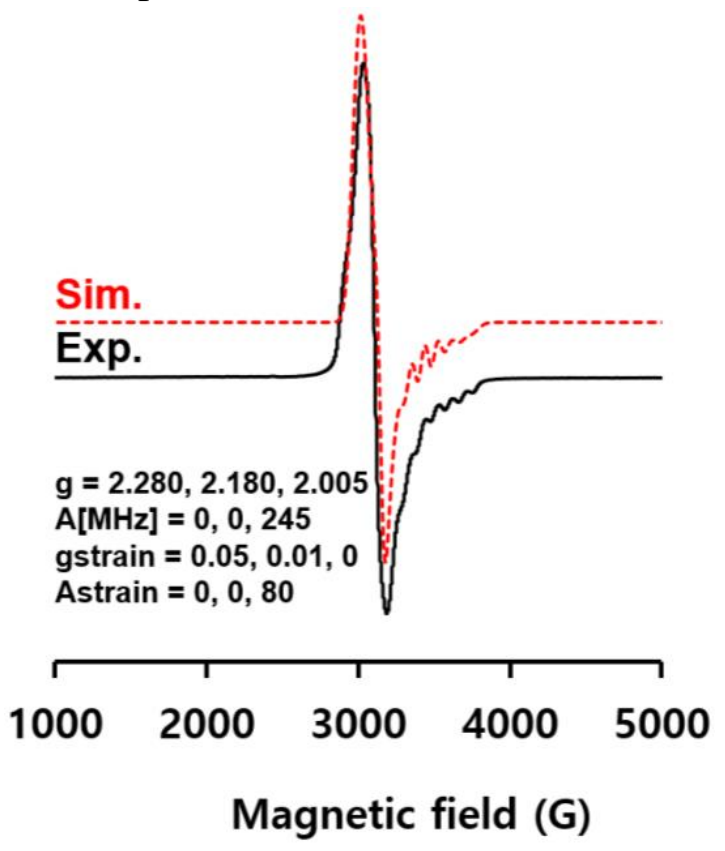

Figure S46. X-Band EPR spectrum of $\left\{\left({ }^{\text {acriPNP }}\right) \mathrm{Co}(\right.$ biph $\left.)\right\}\{\mathrm{K}(18-\mathrm{C}-6)\}(8)$ in toluene (black) at $10 \mathrm{~K}$ with a simulated spectrum (red).

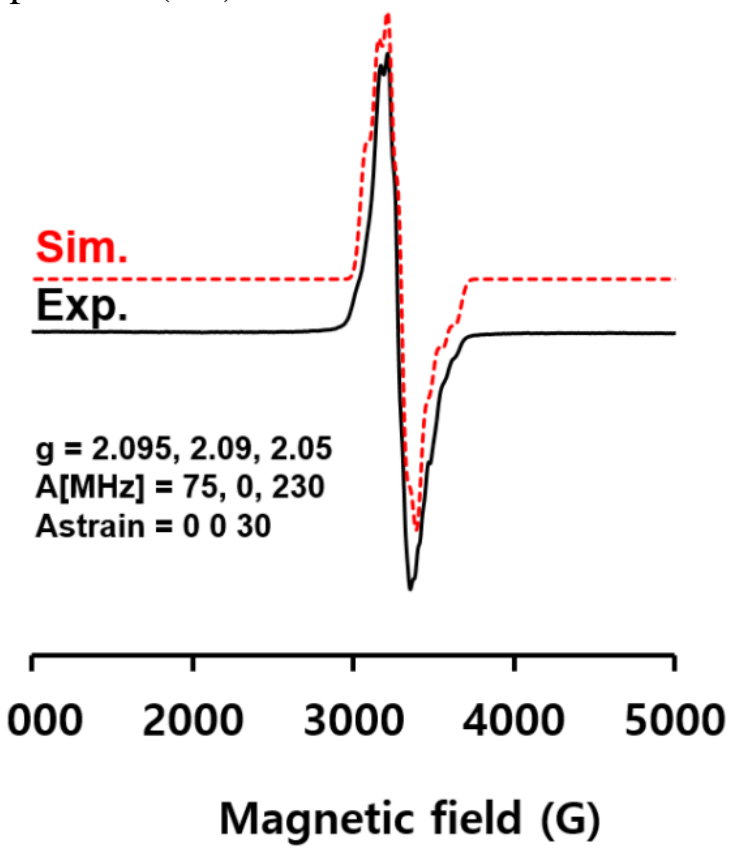


Figure S47. Normalized Co K-edge XAS data of 2-bzq (black), 4-bpy (red), 4-phen (blue) and $\mathbf{6}$ (green). The top inset depicts the first derivative spectra and the first inflection points of the rising edge region are marked with an asterisk.

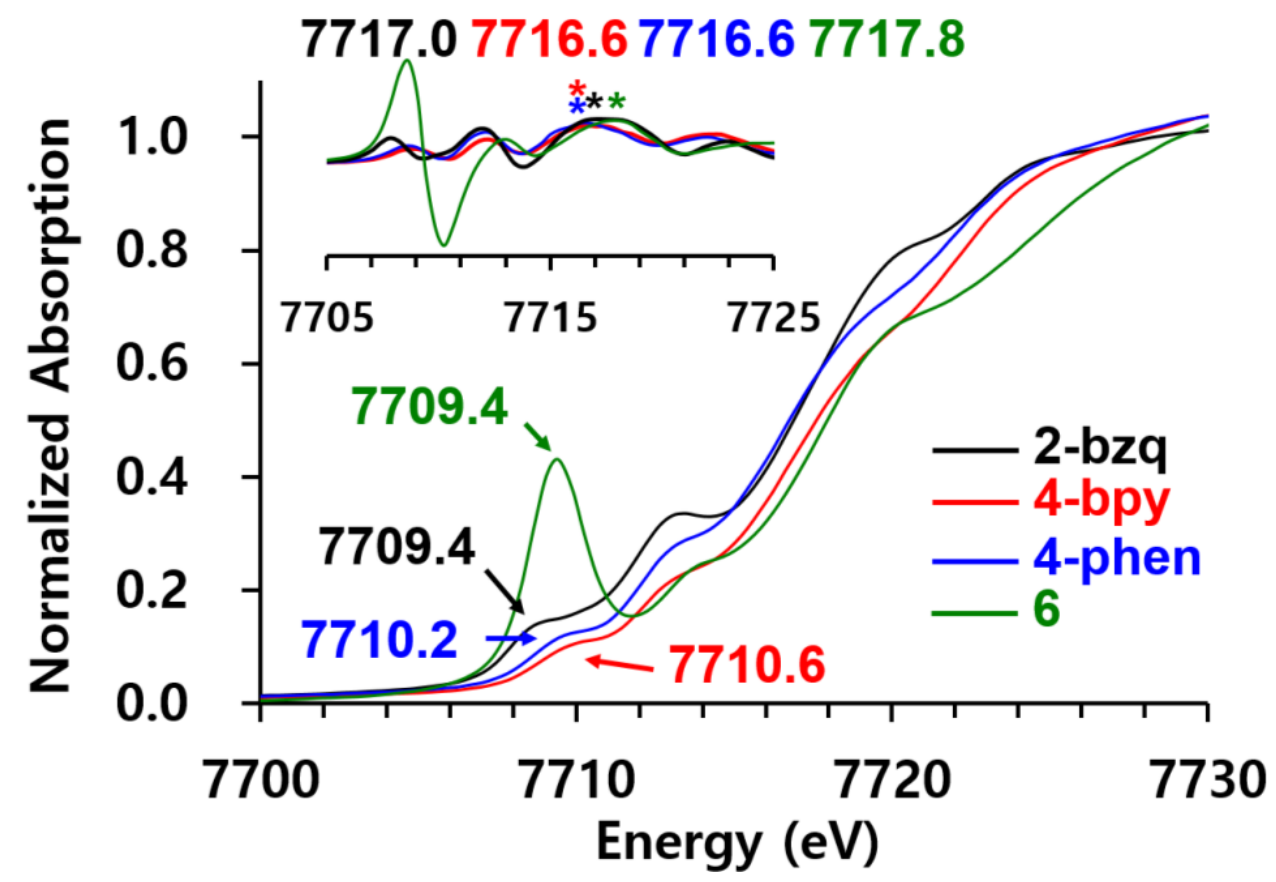


Figure S48. Cyclic voltammogram of 3-N2 (black), 2-ppy (red), 2-bzq (blue), 4-bpy (green), 4-phen (purple) and 6 (brown) with scan rate $100 \mathrm{mV} / \mathrm{s}$. The redox couples vs. Fc/Fc+ were observed in THF with $0.3 \mathrm{M}$ tetra-n-butylammonium hexafluorophosphate as an electrolyte.

Open circuit potential

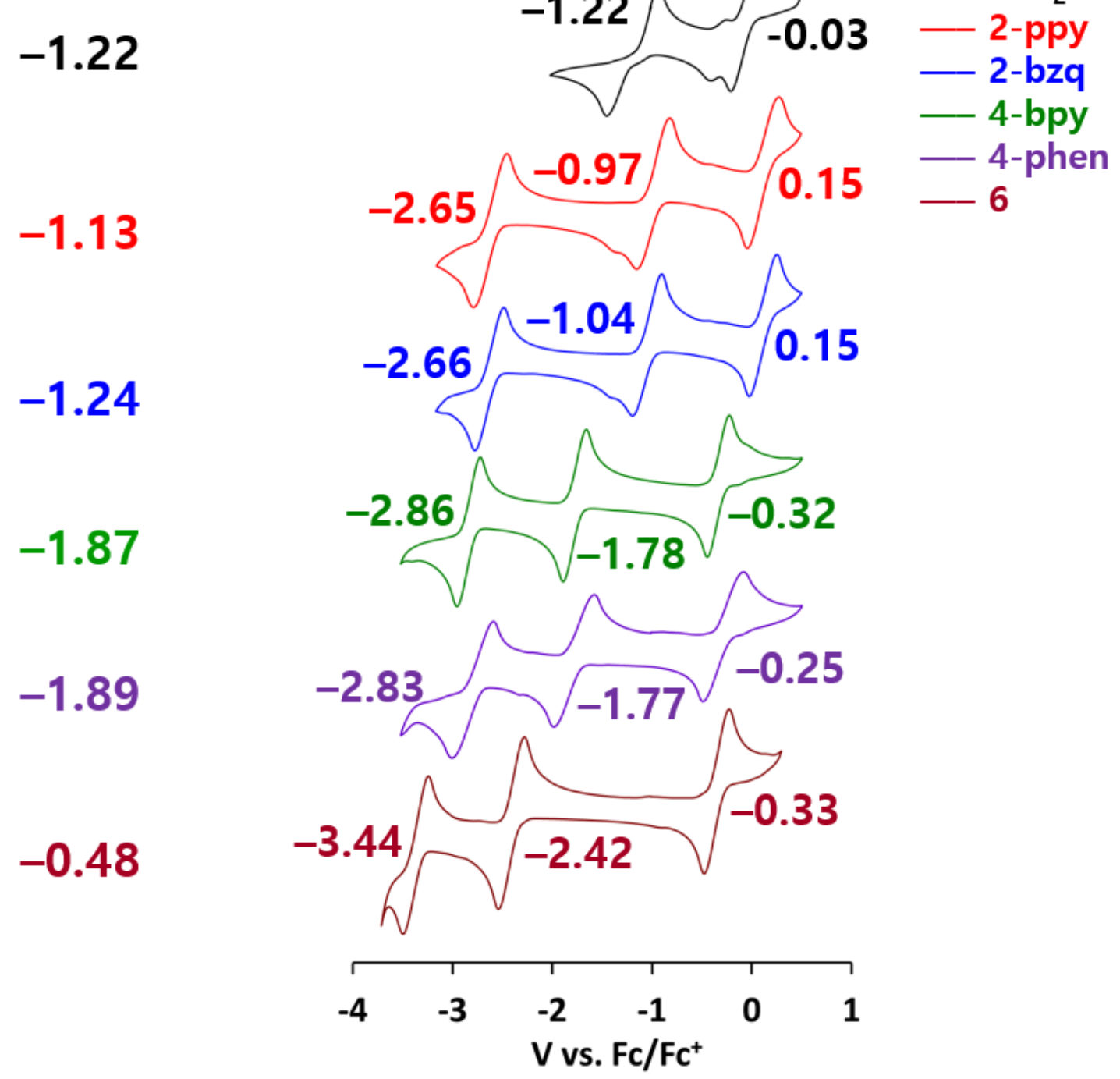


Figure S49. Cyclic voltammogram of 2-ppy in $0.3 \mathrm{M}$ tetra-n-butylammonium hexafluorophosphate/THF after 1st (black), 50th (red) and 100th (blue) cycle at a sweeping rate of $50 \mathrm{mV} / \mathrm{s}$.

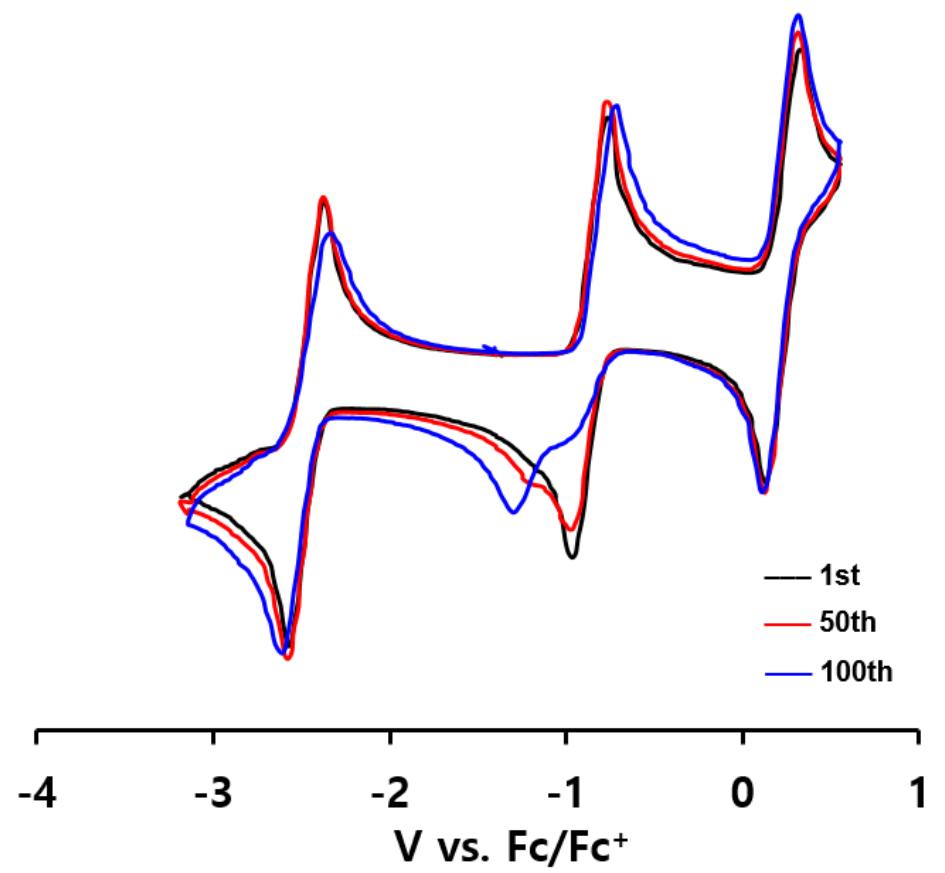

Figure S50. Cyclic voltammogram of 2-bzq in $0.3 \mathrm{M}$ tetra-n-butylammonium hexafluorophosphate/THF after 1st (black), 50th (red) and 100th (blue) cycle at a sweeping rate of $50 \mathrm{mV} / \mathrm{s}$.

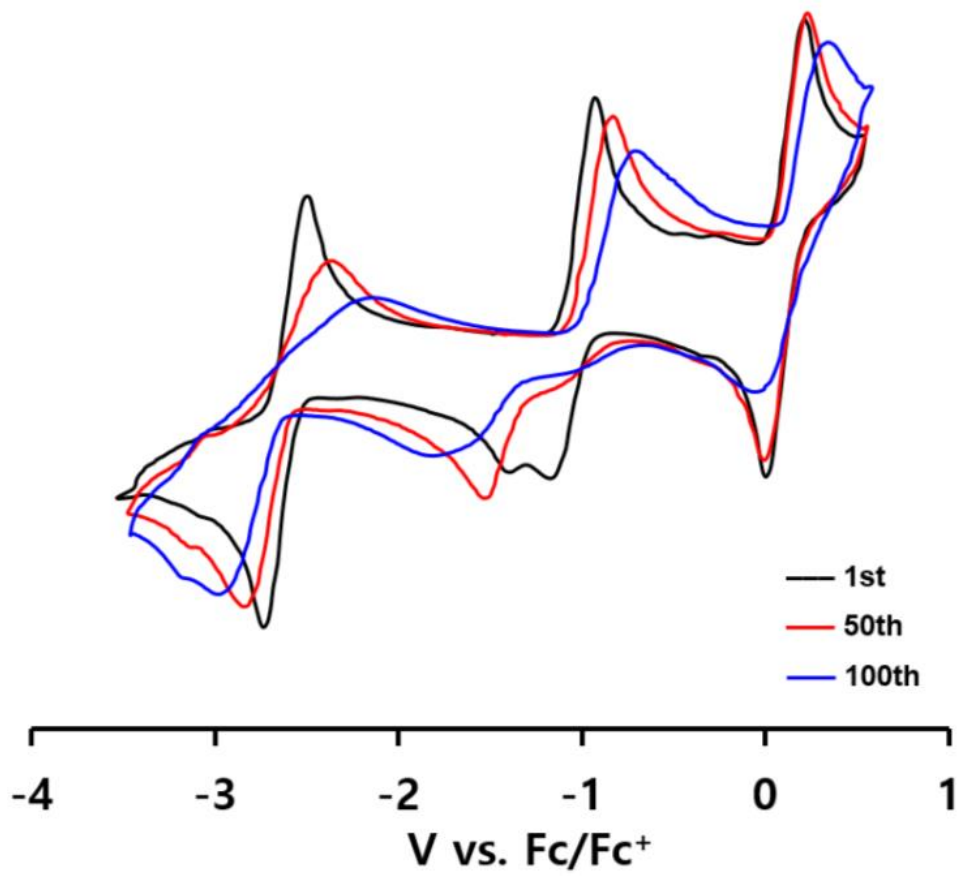


Figure S51. Cyclic voltammogram of 4-bpy in $0.3 \mathrm{M}$ tetra-n-butylammonium hexafluorophosphate/THF after 1st (black), 50th (red) and 100th (blue) cycle at a sweeping rate of $50 \mathrm{mV} / \mathrm{s}$.

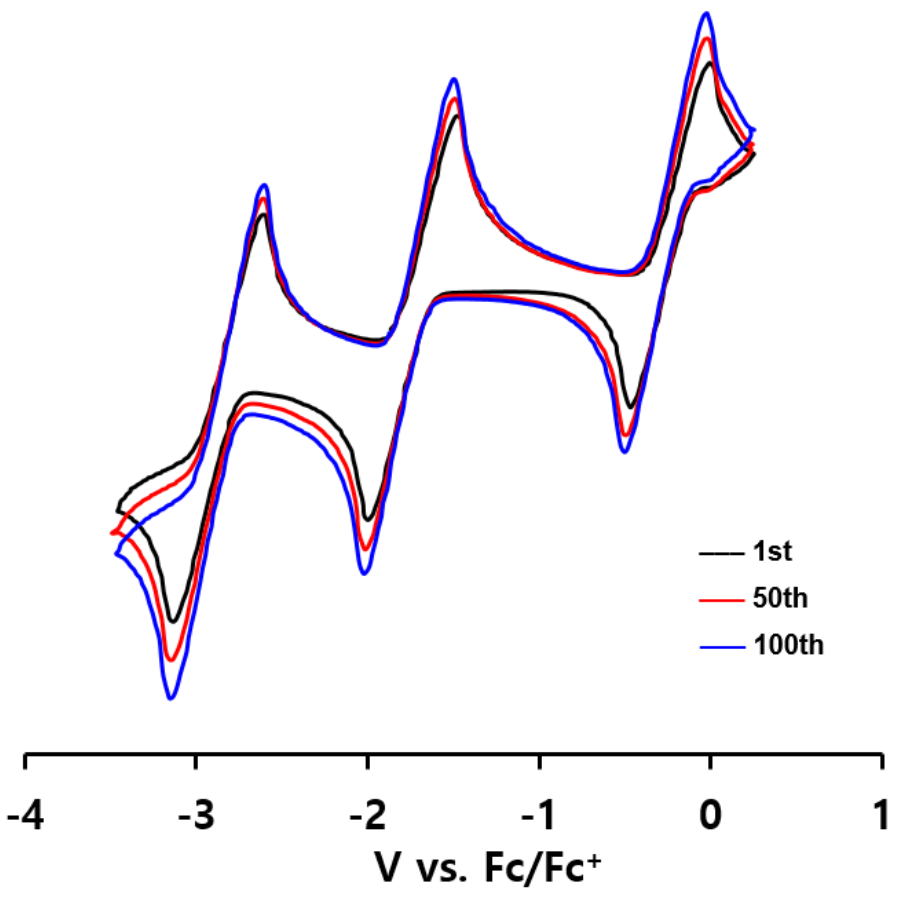

Figure S52. Cyclic voltammogram of 4-phen in $0.3 \mathrm{M}$ tetra-n-butylammonium hexafluorophosphate/THF after 1st (black), 50th (red) and 100th (blue) cycle at a sweeping rate of $50 \mathrm{mV} / \mathrm{s}$.

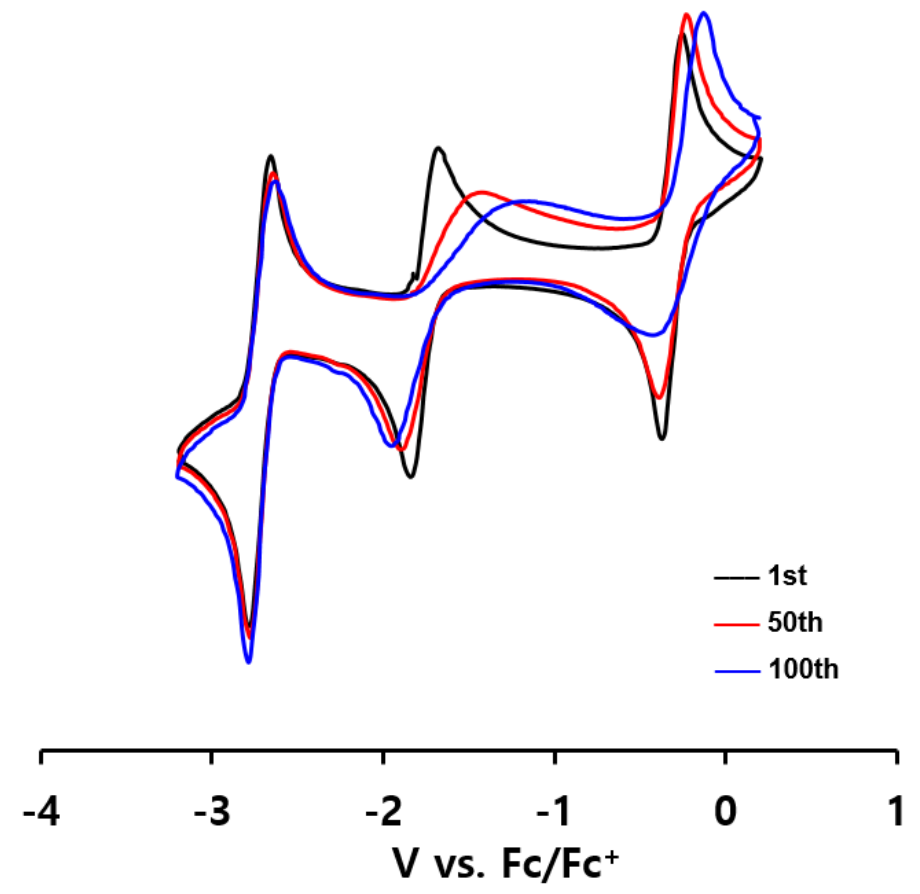


Figure S53. Cyclic voltammogram of $\mathbf{6}$ in $0.3 \mathrm{M}$ tetra-n-butylammonium hexafluorophosphate/THF after 1st (black), 50th (red) and 100th (blue) cycle at a sweeping rate of $50 \mathrm{mV} / \mathrm{s}$.

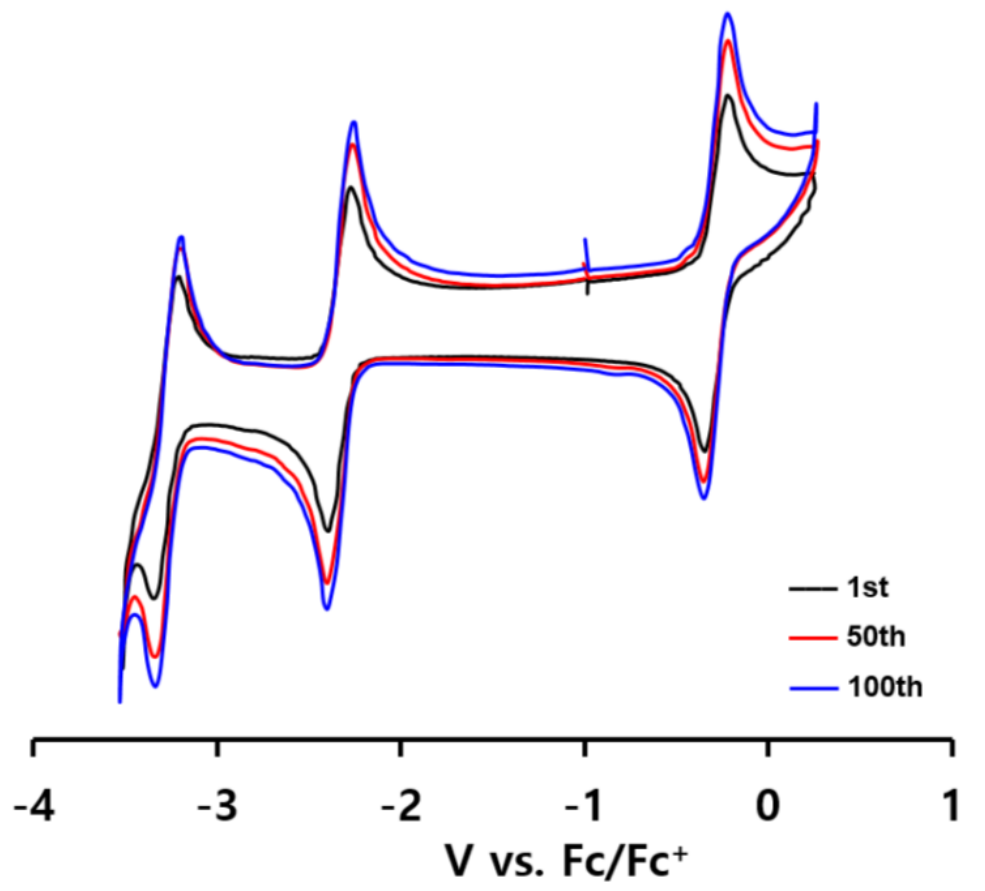

Figure S54. Cyclic voltammogram of 2-ppy in the presence of fc in $0.3 \mathrm{M}$ tetra-nbutylammonium hexafluorophosphate/THF after 1st (black), 50th (red) and 100th (blue) cycle at a sweeping rate of $50 \mathrm{mV} / \mathrm{s}$.

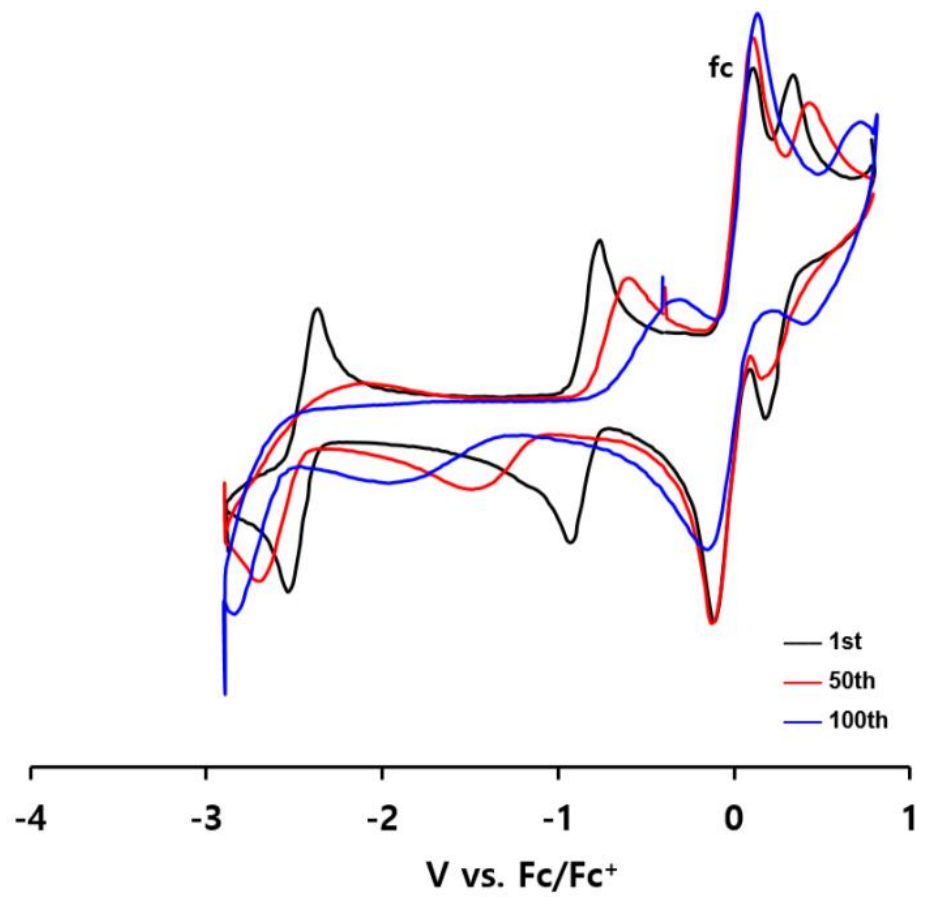


Figure S55. Cyclic voltammogram of 4-bpy in the presence of fc in $0.3 \mathrm{M}$ tetra-nbutylammonium hexafluorophosphate/THF after 1st (black), 50th (red) and 100th (blue) cycle at a sweeping rate of $50 \mathrm{mV} / \mathrm{s}$.

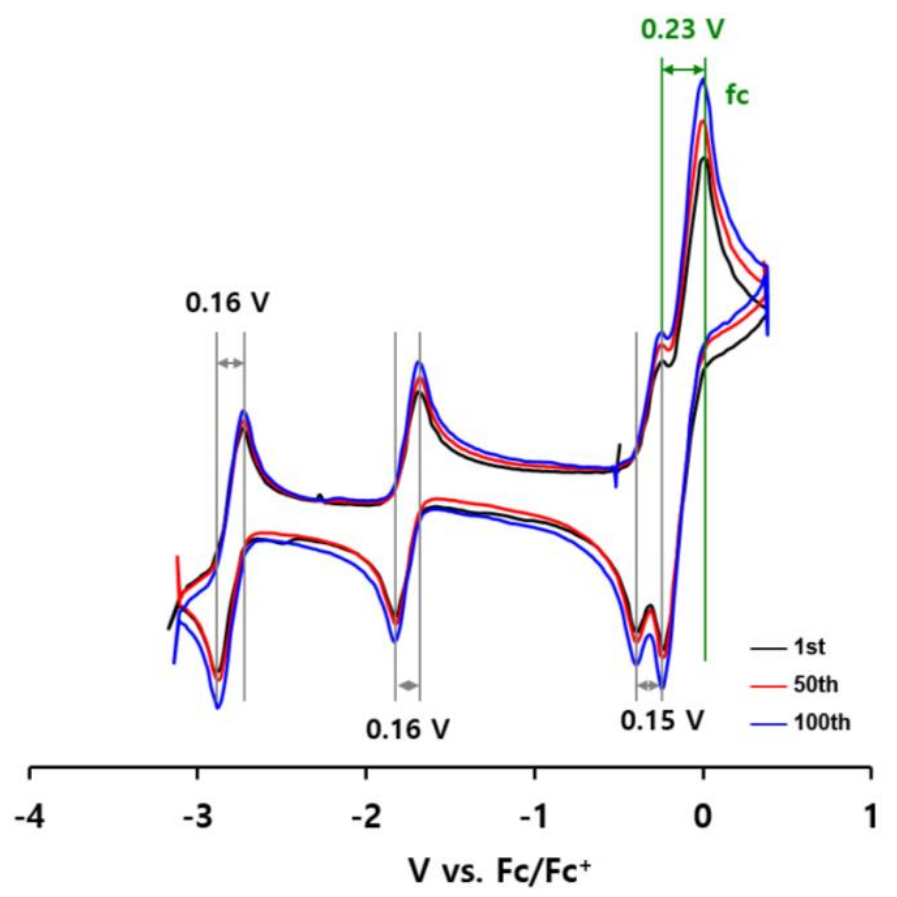

Figure S56. Cyclic voltammogram of $\mathbf{6}$ in the presence of fc in $0.3 \mathrm{M}$ tetra-n-butylammonium hexafluorophosphate/THF after 1st (black), 50th (red) and 100th (blue) cycle at a sweeping rate of $50 \mathrm{mV} / \mathrm{s}$.

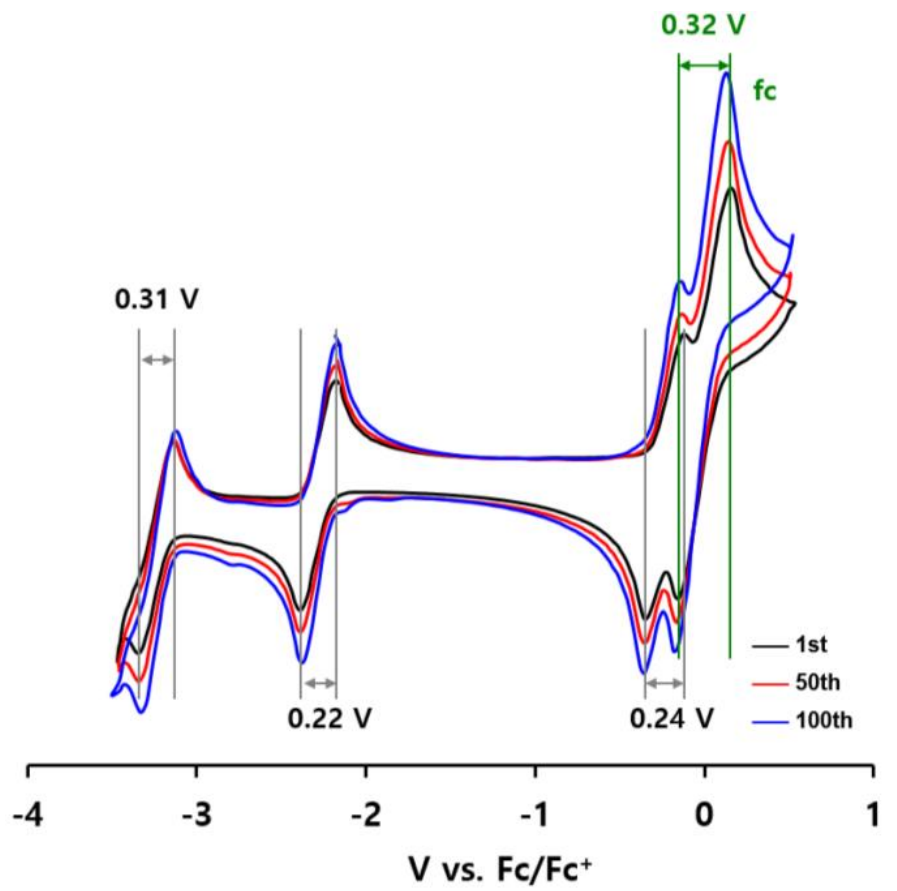


Table S11. Solvent evaporation effect over the CV cycling test of 4-bpy in the presence of Fc; current and relative current over cycles.

\begin{tabular}{|c|c|c|c|c|c|c|c|c|}
\hline $\begin{array}{r}\text { Cathodic } \\
\text { current }\end{array}$ & $\begin{array}{c}\text { At } \\
-2.86 \mathrm{~V}\end{array}$ & $\begin{array}{c}\text { Ratio } n^{\text {th }} \\
\text { vs } 1^{\text {st }} \\
\text { cycle } \\
\end{array}$ & $\begin{array}{c}\text { At } \\
-1.78 \mathrm{~V}\end{array}$ & $\begin{array}{c}\text { Ratio } \mathrm{n}^{\text {th }} \\
\mathrm{vs}^{\mathrm{st}} \\
\text { cycle } \\
\end{array}$ & $\begin{array}{c}\text { At } \\
-0.32 \mathrm{~V}\end{array}$ & $\begin{array}{c}\text { Ratio } \mathrm{n}^{\text {th }} \\
\text { vs } 1^{\text {st }} \\
\text { cycle } \\
\end{array}$ & $\begin{array}{c}\text { At } \\
0.00 \mathrm{~V} \\
\text { for } \mathrm{Fc}\end{array}$ & $\begin{array}{c}\text { Ratio } \mathrm{n}^{\text {th }} \\
\text { vs } 1^{\text {st }} \\
\text { cycle } \\
\end{array}$ \\
\hline $1 \mathrm{st}$ & 0.0127 & 1.00 & 0.0184 & 1.00 & 0.0232 & 1.00 & 0.0549 & 1.00 \\
\hline 50th & 0.0140 & 1.10 & 0.0206 & 1.12 & 0.0258 & 1.11 & 0.0605 & 1.10 \\
\hline 100th & 0.0154 & 1.21 & 0.0231 & 1.25 & 0.0277 & 1.19 & 0.0672 & 1.22 \\
\hline $\begin{array}{l}\text { Anodic } \\
\text { current }\end{array}$ & $\begin{array}{c}\mathrm{At} \\
-2.86 \mathrm{~V}\end{array}$ & $\begin{array}{c}\text { Ratio } n^{\text {th }} \\
\text { vs } 1^{\text {st }} \\
\text { cycle }\end{array}$ & $\begin{array}{c}\text { At } \\
-1.78 \mathrm{~V}\end{array}$ & $\begin{array}{c}\text { Ratio } n^{\text {th }} \\
\text { vs } 1^{\text {st }} \\
\text { cycle }\end{array}$ & $\begin{array}{c}\text { At } \\
-0.32 \mathrm{~V}\end{array}$ & $\begin{array}{c}\text { Ratio } n^{\text {th }} \\
\text { vs } 1^{\text {st }} \\
\text { cycle }\end{array}$ & $\begin{array}{c}\text { At } \\
0.00 \mathrm{~V} \\
\text { for } \mathrm{Fc}\end{array}$ & $\begin{array}{c}\text { Ratio } n^{\text {th }} \\
\text { vs } 1^{\text {st }} \\
\text { cycle }\end{array}$ \\
\hline $1 \mathrm{st}$ & -0.025 & 1.00 & -0.0167 & 1.00 & -0.0190 & 1.00 & -0.0217 & 1.00 \\
\hline 50 th & -0.0263 & 1.04 & -0.0178 & 1.07 & -0.0228 & 1.20 & -0.0228 & 1.05 \\
\hline 100 th & -0.0309 & 1.22 & -0.0205 & 1.23 & -0.0242 & 1.27 & -0.0278 & 1.28 \\
\hline
\end{tabular}

Table S12. Solvent evaporation effect over the CV cycling test of 6 in the presence of Fc; current and relative current over cycles.

\begin{tabular}{|c|c|c|c|c|c|c|c|c|}
\hline $\begin{array}{l}\text { Cathodic } \\
\text { current }\end{array}$ & $\begin{array}{c}\text { At } \\
-3.44 \mathrm{~V}\end{array}$ & $\begin{array}{c}\text { Ratio } n^{\text {th }} \\
\text { vs } 1^{\text {st }} \\
\text { cycle }\end{array}$ & $\begin{array}{c}\text { At } \\
-2.42 \mathrm{~V}\end{array}$ & $\begin{array}{c}\text { Ratio } \mathrm{n}^{\text {th }} \\
\text { vs } 1^{\text {st }} \\
\text { cycle }\end{array}$ & $\begin{array}{c}\mathrm{At} \\
-0.33 \mathrm{~V}\end{array}$ & $\begin{array}{c}\text { Ratio } n^{\text {th }} \\
\text { vs } 1^{\text {st }} \\
\text { cycle }\end{array}$ & $\begin{array}{c}\text { At } \\
0.00 \mathrm{~V} \\
\text { for Fc }\end{array}$ & $\begin{array}{c}\text { Ratio } \mathrm{n}^{\text {th }} \\
\text { vs } 1^{\text {st }} \\
\text { cycle }\end{array}$ \\
\hline $1 \mathrm{st}$ & 0.00704 & 1.00 & 0.0202 & 1.00 & 0.0291 & 1.00 & 0.0594 & 1.00 \\
\hline 50th & 0.00736 & 1.05 & 0.0227 & 1.12 & 0.0333 & 1.14 & 0.0689 & 1.16 \\
\hline 100th & 0.00902 & 1.28 & 0.0288 & 1.42 & 0.0399 & 1.37 & 0.0833 & 1.40 \\
\hline $\begin{array}{l}\text { Anodic } \\
\text { current }\end{array}$ & $\begin{array}{c}\text { At } \\
-3.44 \mathrm{~V}\end{array}$ & $\begin{array}{c}\text { Ratio } \mathrm{n}^{\text {th }} \\
\text { vs } 1^{\text {st }} \\
\text { cycle }\end{array}$ & $\begin{array}{c}\text { At } \\
-2.42 \mathrm{~V}\end{array}$ & $\begin{array}{c}\text { Ratio } n^{\text {th }} \\
{\text { vs } 1^{\text {st }}}^{\text {cycle }} \\
\end{array}$ & $\begin{array}{c}\text { At } \\
-0.33 \mathrm{~V}\end{array}$ & $\begin{array}{c}\text { Ratio } n^{\text {th }} \\
\text { vs } 1^{\text {st }} \\
\text { cycle }\end{array}$ & $\begin{array}{c}\text { At } \\
0.00 \mathrm{~V} \\
\text { for } \mathrm{Fc}\end{array}$ & $\begin{array}{c}\text { Ratio } \mathrm{n}^{\text {th }} \\
\text { vs } 1^{\text {st }} \\
\text { cycle }\end{array}$ \\
\hline $1 \mathrm{st}$ & -0.0386 & 1.00 & -0.0278 & 1.00 & -0.0256 & 1.00 & -0.0297 & 1.00 \\
\hline 50th & -0.0404 & 1.05 & -0.0324 & 1.16 & -0.0304 & 1.19 & -0.0346 & 1.17 \\
\hline 100th & -0.0492 & 1.27 & -0.0389 & 1.40 & -0.0366 & 1.43 & -0.0411 & 1.39 \\
\hline
\end{tabular}


Table S13. Peak-current ratio $\left(\mathrm{i}_{\mathrm{p} a} / \mathrm{i}_{\mathrm{pc}}\right)$ acquired from Figure S50 and Figure S52.

\begin{tabular}{ccccccc}
\hline 4-bpy & $-2.86 \mathrm{~V}$ & Decay & $-1.78 \mathrm{~V}$ & Decay & $-0.32 \mathrm{~V}$ & Decay \\
\hline $\mathrm{i}_{\mathrm{pa}} / \mathrm{i}_{\mathrm{ic}}(1 \mathrm{st})$ & 1.02 & & 0.85 & & 1.17 & \\
$\mathrm{i}_{\mathrm{pa}} / \mathrm{i}_{\mathrm{pc}}(100 \mathrm{th})$ & 1.01 & $1 \%$ & 0.78 & $12 \%$ & 1.08 & $8 \%$ \\
\hline $\mathbf{6}$ & $-3.44 \mathrm{~V}^{*}$ & Decay & $-2.42 \mathrm{~V}$ & Decay & $-0.33 \mathrm{~V}$ & Decay \\
\hline $\mathrm{i}_{\mathrm{pa}} / \mathrm{i}_{\mathrm{pc}}(1 \mathrm{st})$ & 0.43 & & 0.94 & & 1.10 & $5 \%$ \\
$\mathrm{i}_{\mathrm{pa}} / \mathrm{i}_{\mathrm{pc}}(100 \mathrm{th})$ & 0.71 & - & 0.93 & & 1.05 & \\
\hline
\end{tabular}

*The reduction occurs at the boundary of electrochemical window, thus the appropriate $\mathrm{i}_{\mathrm{pa}} / \mathrm{i}_{\mathrm{pc}}$ could not obtained.

Figure S57. Electronic structures for (acriPNP)Co(ppy) (2-ppy) derived from the geometry optimization and frequency calculations using unrestricted B3LYP level of DFT; energies in $\mathrm{cm}^{-1}$. Lobal representations correspond to the orbitals indicated by the number with 0.05 isocontours.
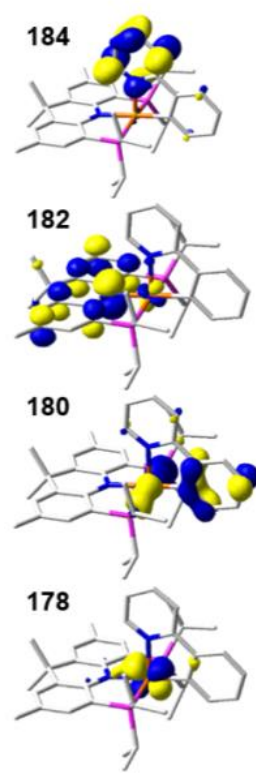

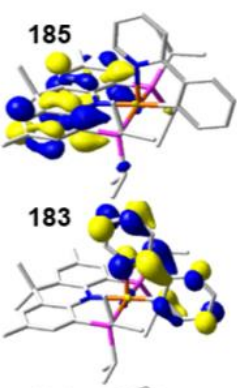

181

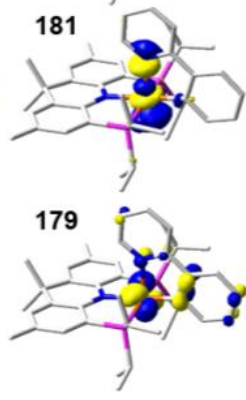

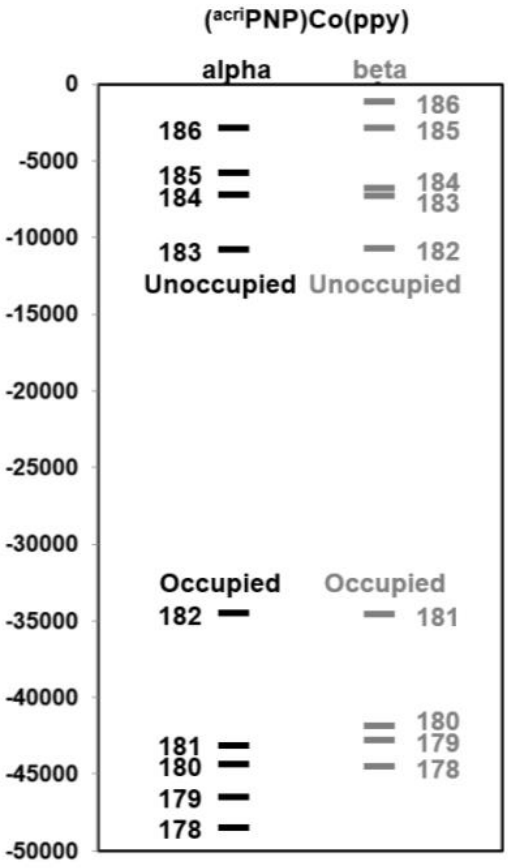
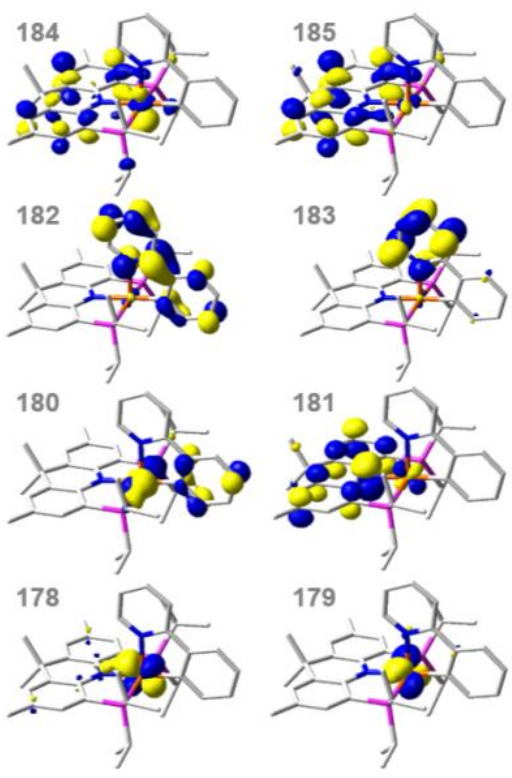
Figure S58. Electronic structures for isomer of 2-ppy (2-ppy') derived from the geometry optimization and frequency calculations using unrestricted B3LYP level of DFT; energies in $\mathrm{cm}^{-1}$. Lobal representations correspond to the orbitals indicated by the number with 0.05 isocontours.

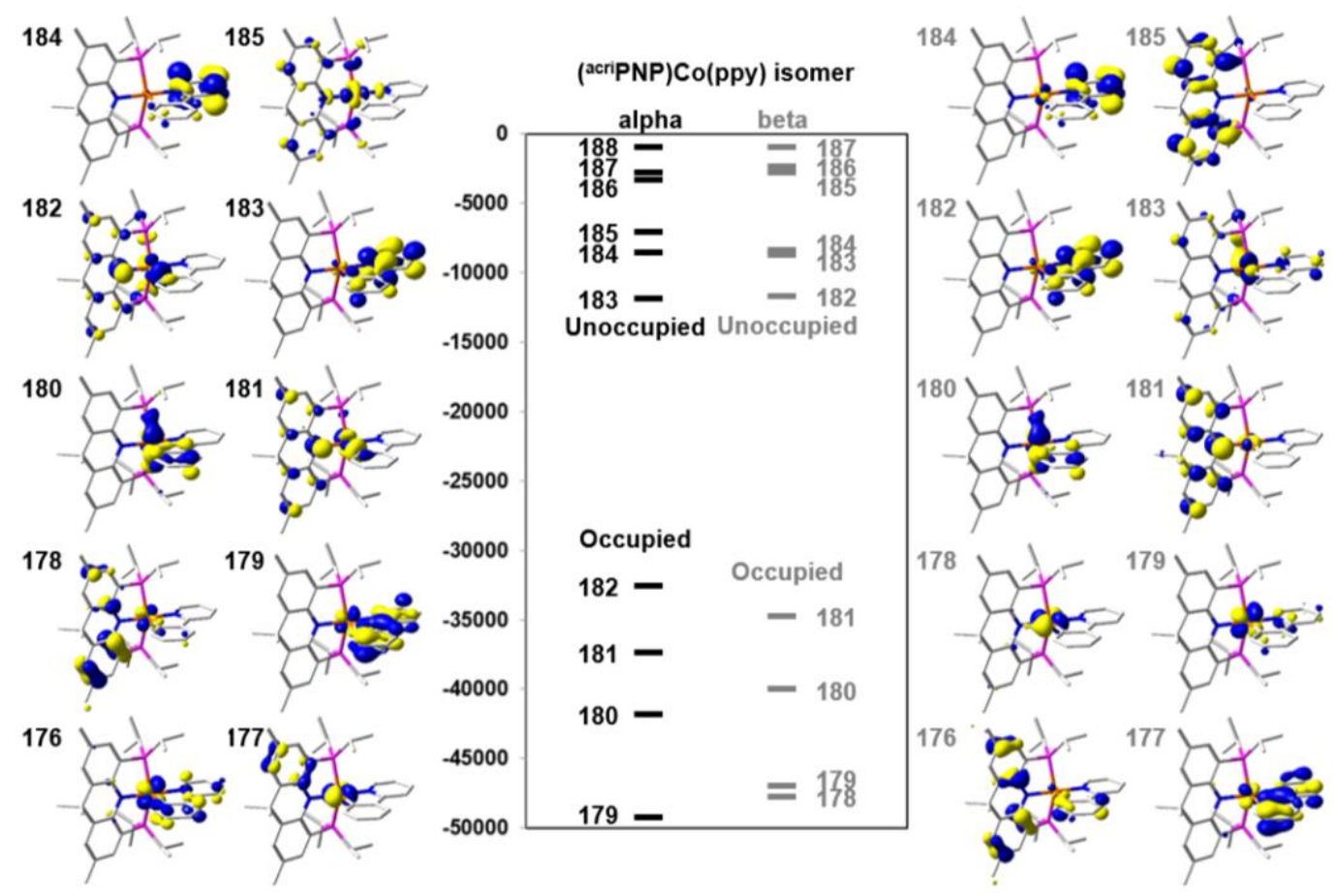

Figure S59. Electronic structures for ( ${ }^{\text {acripNP) }} \mathrm{Co}(\mathrm{bzq})$ (2-bzq) derived from the geometry optimization and frequency calculations using unrestricted B3LYP level of DFT; energies in $\mathrm{cm}^{-1}$. Lobal representations correspond to the orbitals indicated by the number with 0.05 isocontours.
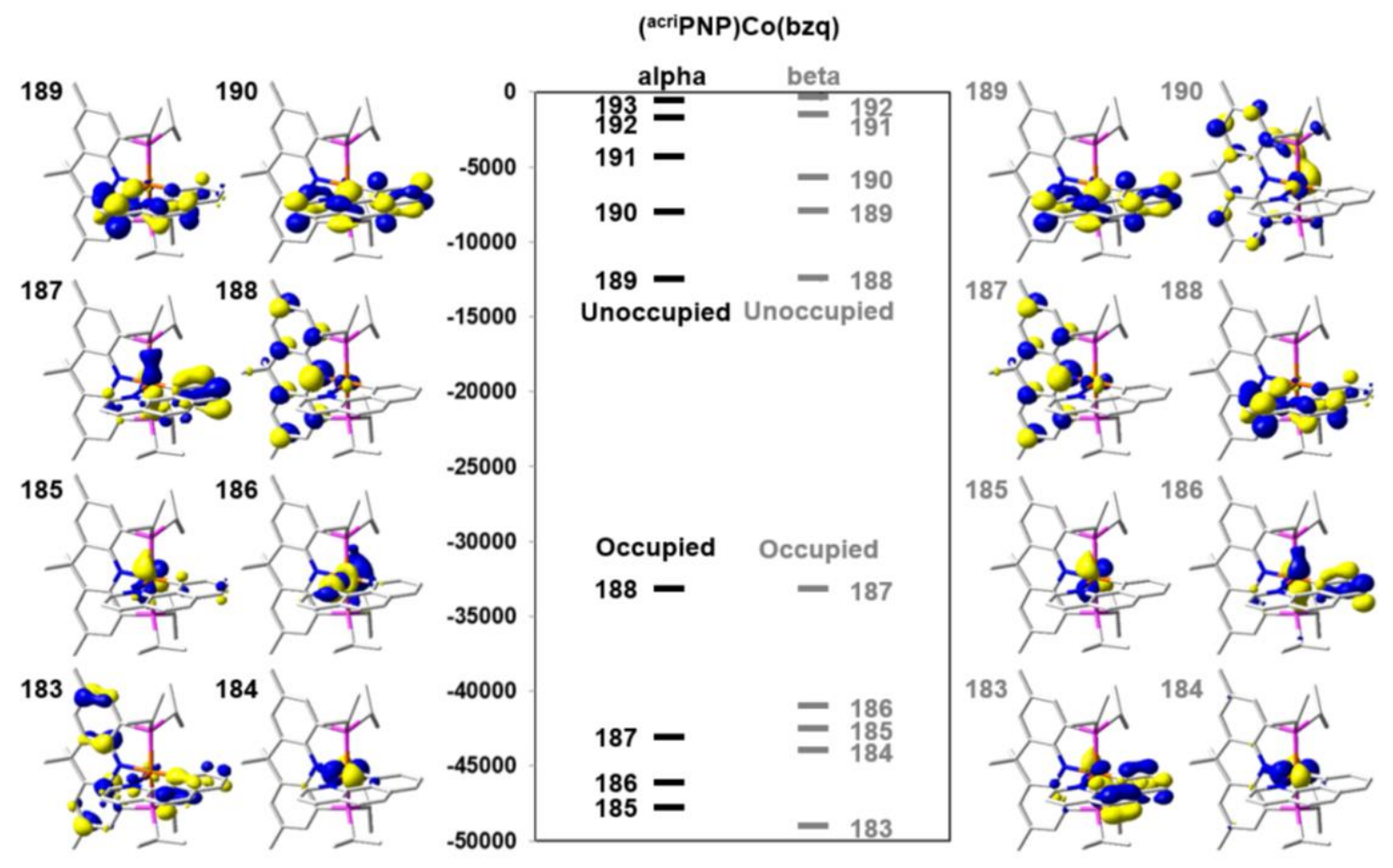
Figure S60. Electronic structures for isomer of 2-bzq (2-bzq') derived from the geometry optimization and frequency calculations using unrestricted B3LYP level of DFT; energies in $\mathrm{cm}^{-1}$. Lobal representations correspond to the orbitals indicated by the number with 0.05 isocontours.

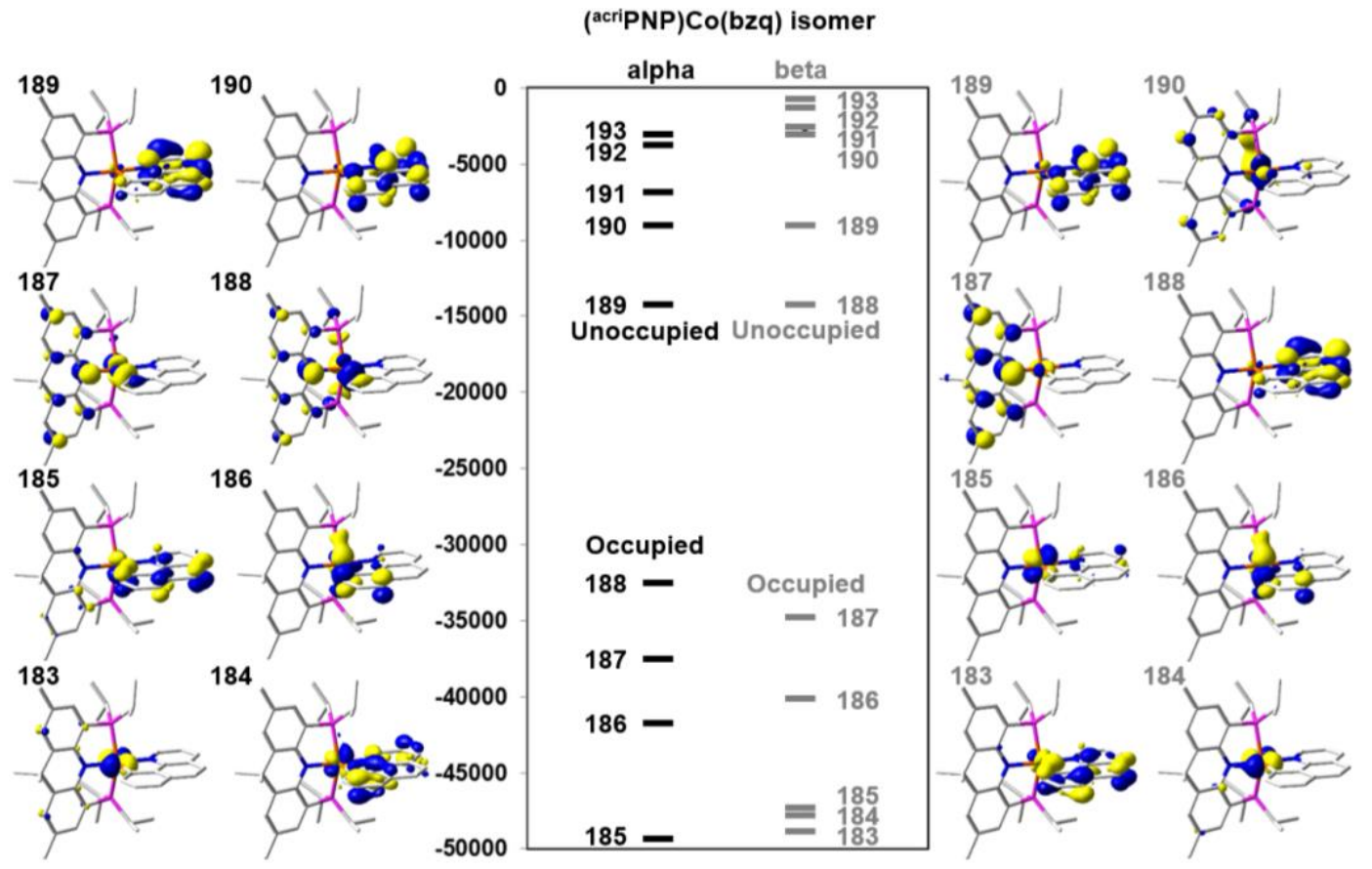

Figure S61. Electronic structures for 2-ppy and 2-bzq derived from the geometry optimization and frequency calculations using restricted open shell B3LYP level of DFT; energies in $\mathrm{cm}^{-1}$. Lobal representations correspond to the orbitals indicated by the number with 0.05 isocontours.
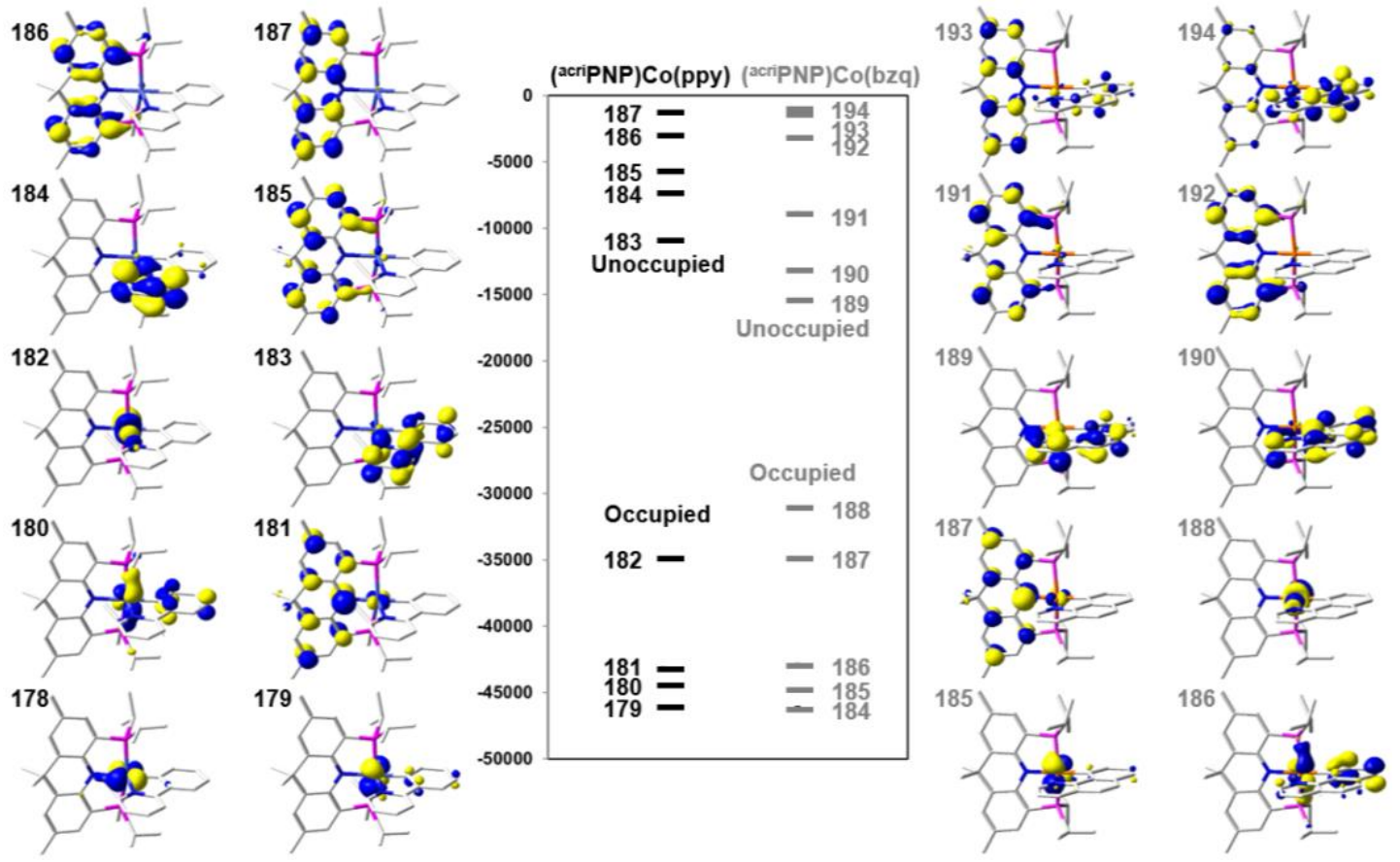
Table S14. Computed energies of (acriPNP)Co(ppy) (2-ppy) and (acriPNP)Co(bzq) (2-bzq) and

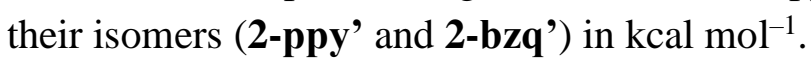

\begin{tabular}{cccccccc}
\hline & Def2-SVP & Def2-TZVPP & $\Delta \mathrm{E}$ & $\Delta \mathrm{Z}_{0}$ & $\Delta \mathrm{E}_{\text {thermal }}$ & $-\mathrm{T} \Delta \mathrm{S}$ & $\Delta \mathrm{G}$ \\
2-ppy & 0 & 0 & 0 & 0 & 0 & 0 & 0 \\
2-ppy & 5.05 & -1.39 & 3.67 & 0.75 & -0.19 & 0.50 & 4.73 \\
\hline & Def2-SVP & Def2-TZVPP & $\Delta \mathrm{E}$ & $\Delta \mathrm{Z}_{0}$ & $\Delta \mathrm{E}_{\text {thermal }}$ & $-\mathrm{T} \Delta \mathrm{S}$ & $\Delta \mathrm{G}$ \\
2-bzq & 0 & 0 & 0 & 0 & 0 & 0 & 0 \\
2-bzq & -0.33 & -0.05 & -0.39 & 0.10 & -0.01 & -0.63 & -0.93 \\
\hline
\end{tabular}

Figure S62. Electronic structures for the open shell singlet state of $\left\{\left({ }^{\text {acriPNP}}\right) \operatorname{Co}(\mathrm{ppy})\right\}^{+}(\mathbf{2}-$ $\mathbf{p p y}^{+}$) derived from the geometry optimization and frequency calculations using unrestricted B3LYP level of DFT; energies in $\mathrm{cm}^{-1}$. Lobal representations correspond to the orbitals indicated by the number with 0.05 isocontours.

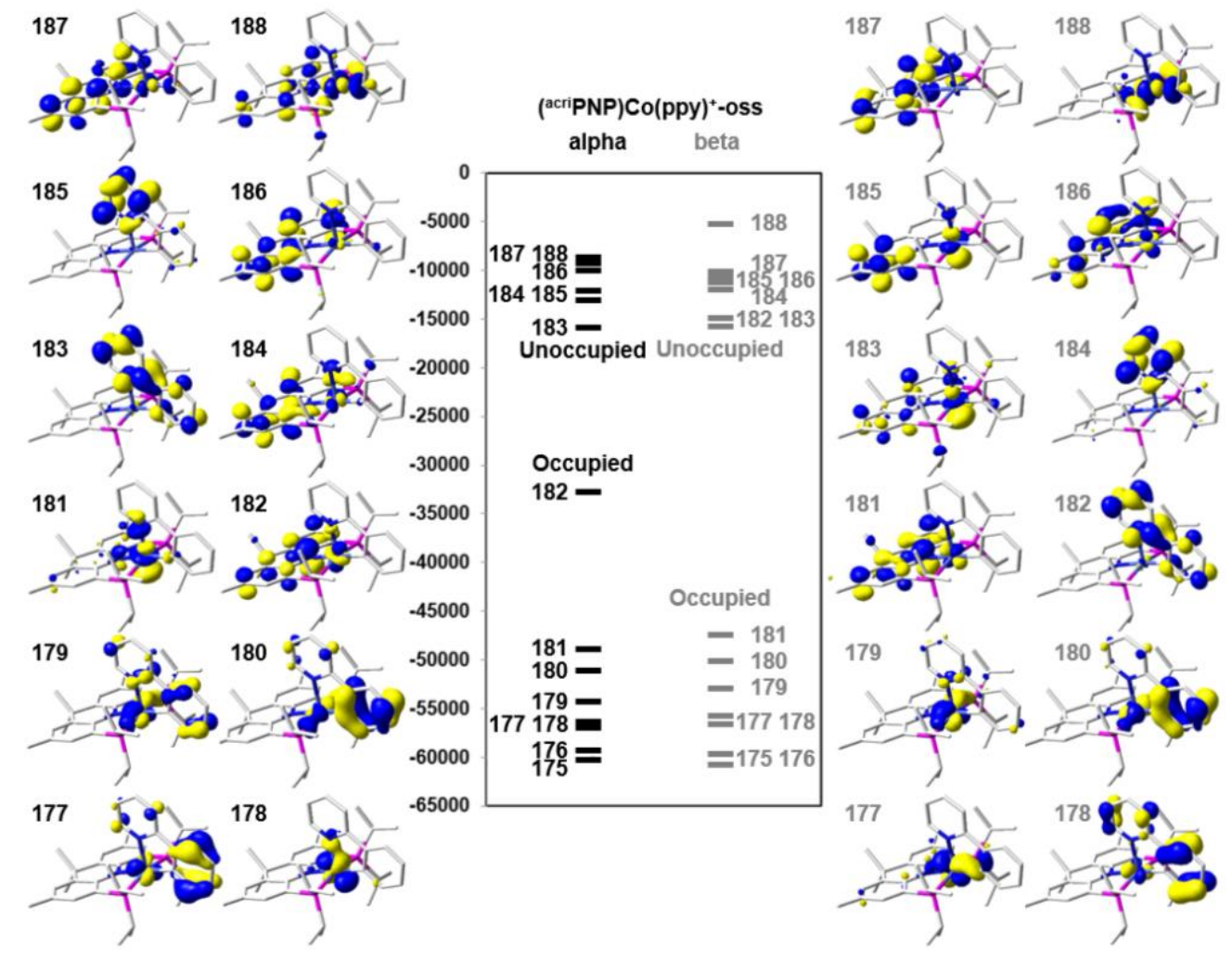


Figure S63. Electronic structures for the open shell triplet state of $\left\{\left({ }^{\text {acriPNP }}\right) \operatorname{Co}(\mathrm{ppy})\right\}^{+}$(2ppy $^{+}$) derived from the geometry optimization and frequency calculations using unrestricted B3LYP level of DFT; energies in $\mathrm{cm}^{-1}$. Lobal representations correspond to the orbitals indicated by the number with 0.05 isocontours.
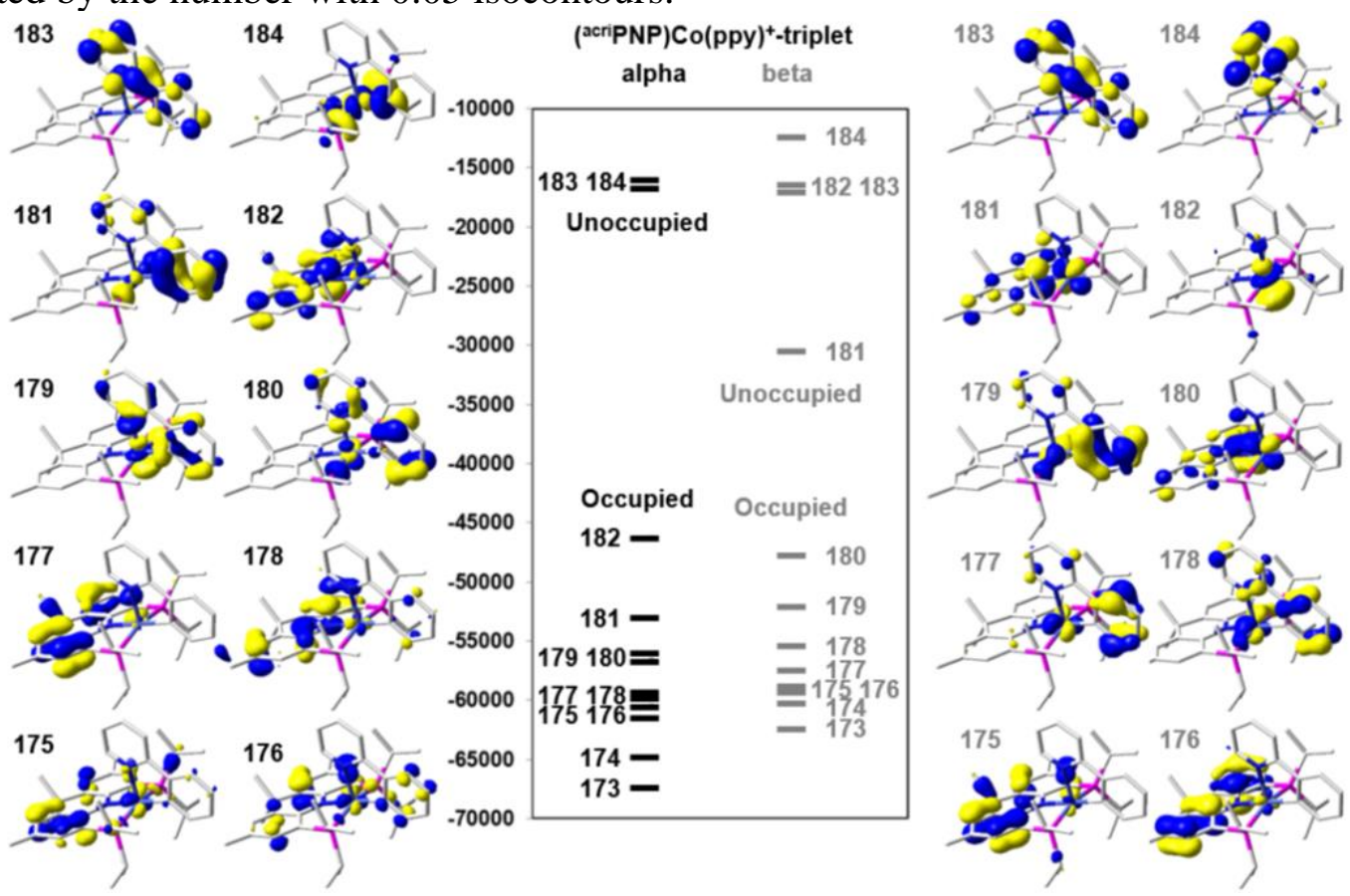

Figure S64. Electronic structures for the closed shell singlet state of $\left\{\left({ }^{\text {acriPNP }}\right) \operatorname{Co}(\mathrm{ppy})\right\}^{+}(\mathbf{2}-$ ppy $\left.^{+}\right)$and $\left\{\left({ }^{\text {acripNP }}\right) \mathrm{Co}(\mathrm{bzq})\right\}^{+}\left(\mathbf{2}-\mathbf{b z q}^{+}\right)$derived from the geometry optimization and frequency calculations using unrestricted B3LYP level of DFT; energies in $\mathrm{cm}^{-1}$. Lobal representations correspond to the orbitals indicated by the number with 0.05 isocontours.

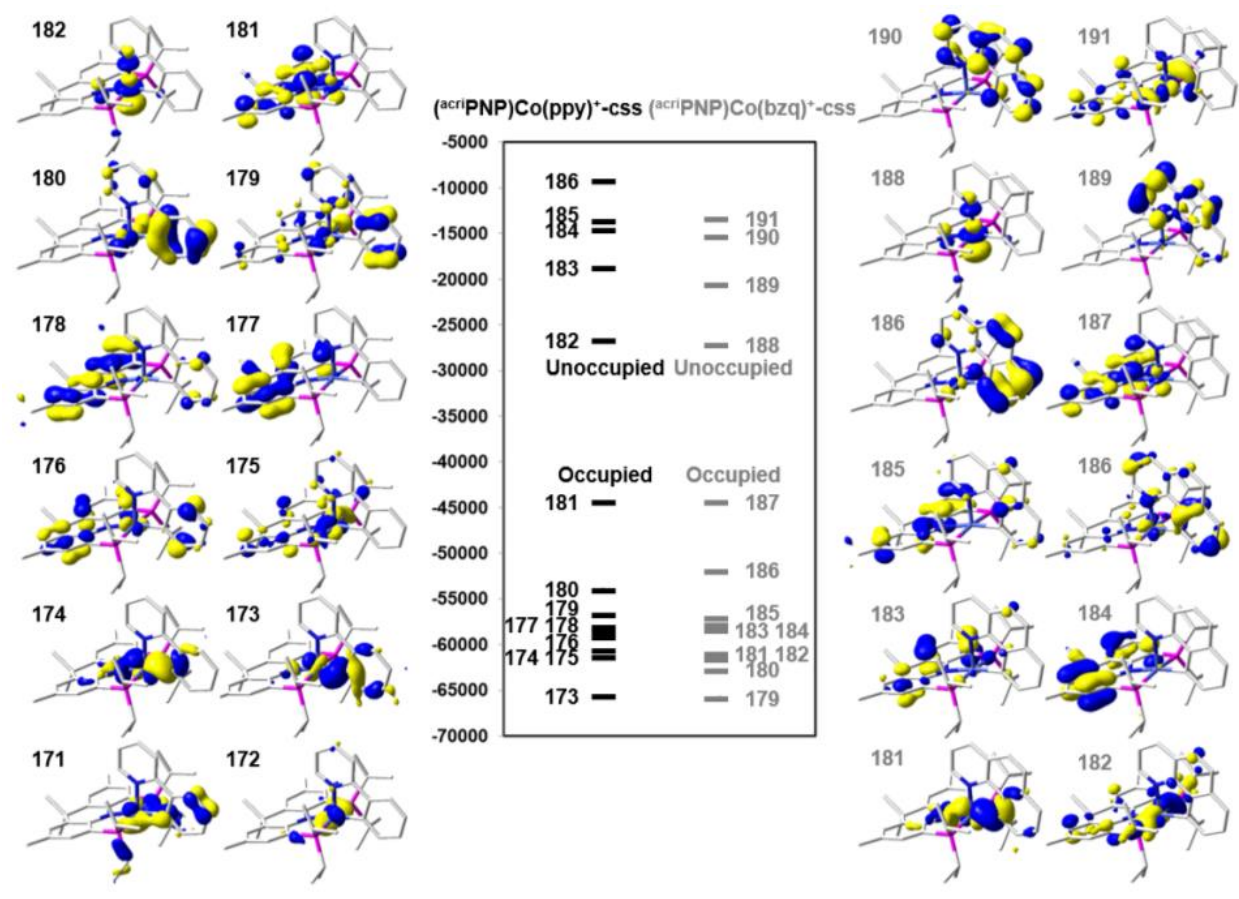


Figure S65. Electronic structures for the open shell singlet state of $\left\{\left({ }^{\text {acriPNP }}\right) \operatorname{Co}(\mathrm{bzq})\right\}^{+}(\mathbf{2}-$ $\mathbf{b z q}^{+}$) derived from the geometry optimization and frequency calculations using unrestricted B3LYP level of DFT; energies in $\mathrm{cm}^{-1}$. Lobal representations correspond to the orbitals indicated by the number with 0.05 isocontours.
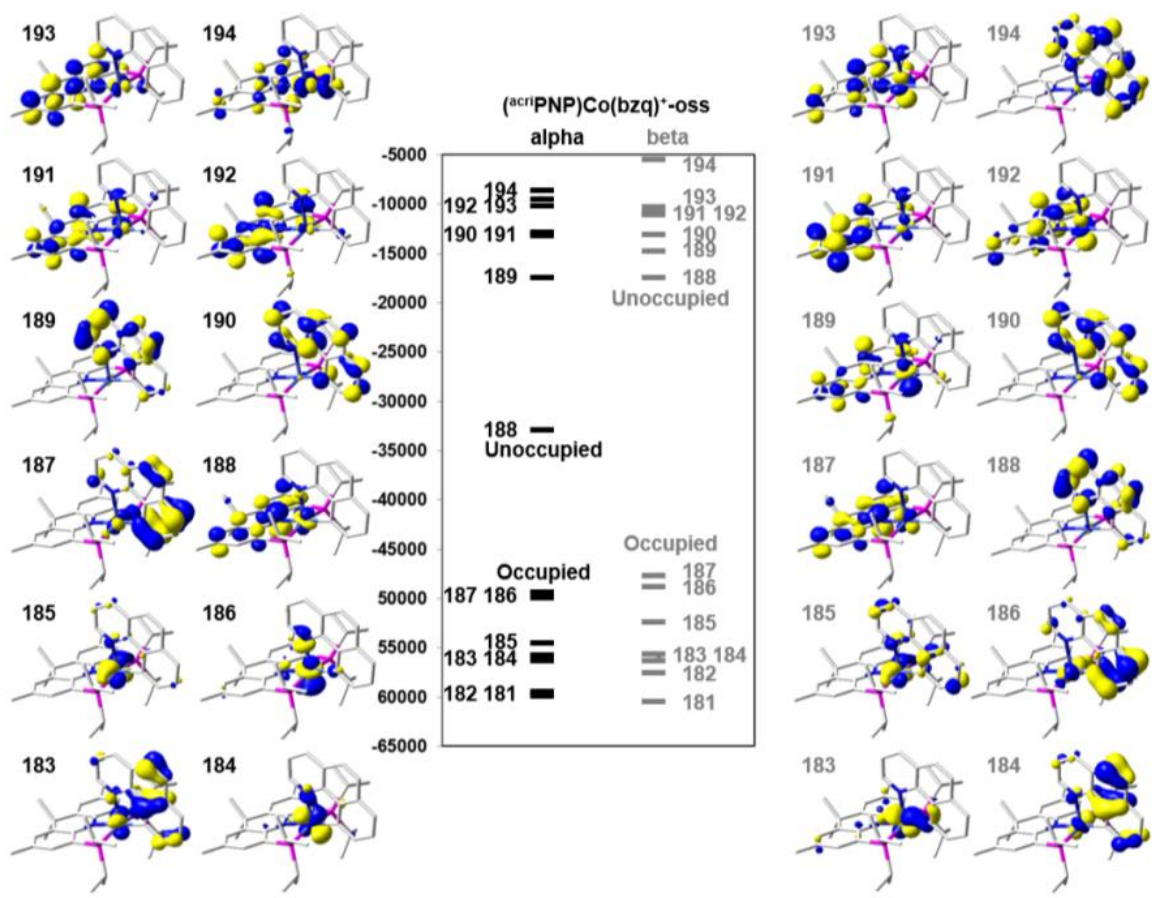

Figure S66. Electronic structures for the open shell triplet state of $\left\{\left({ }^{\text {acriPNP }}\right) \operatorname{Co}(\mathrm{bzq})\right\}^{+}\left(\mathbf{2}-\mathbf{b z q}^{+}\right)$ derived from the geometry optimization and frequency calculations using unrestricted B3LYP level of DFT; energies in $\mathrm{cm}^{-1}$. Lobal representations correspond to the orbitals indicated by the number with 0.05 isocontours.

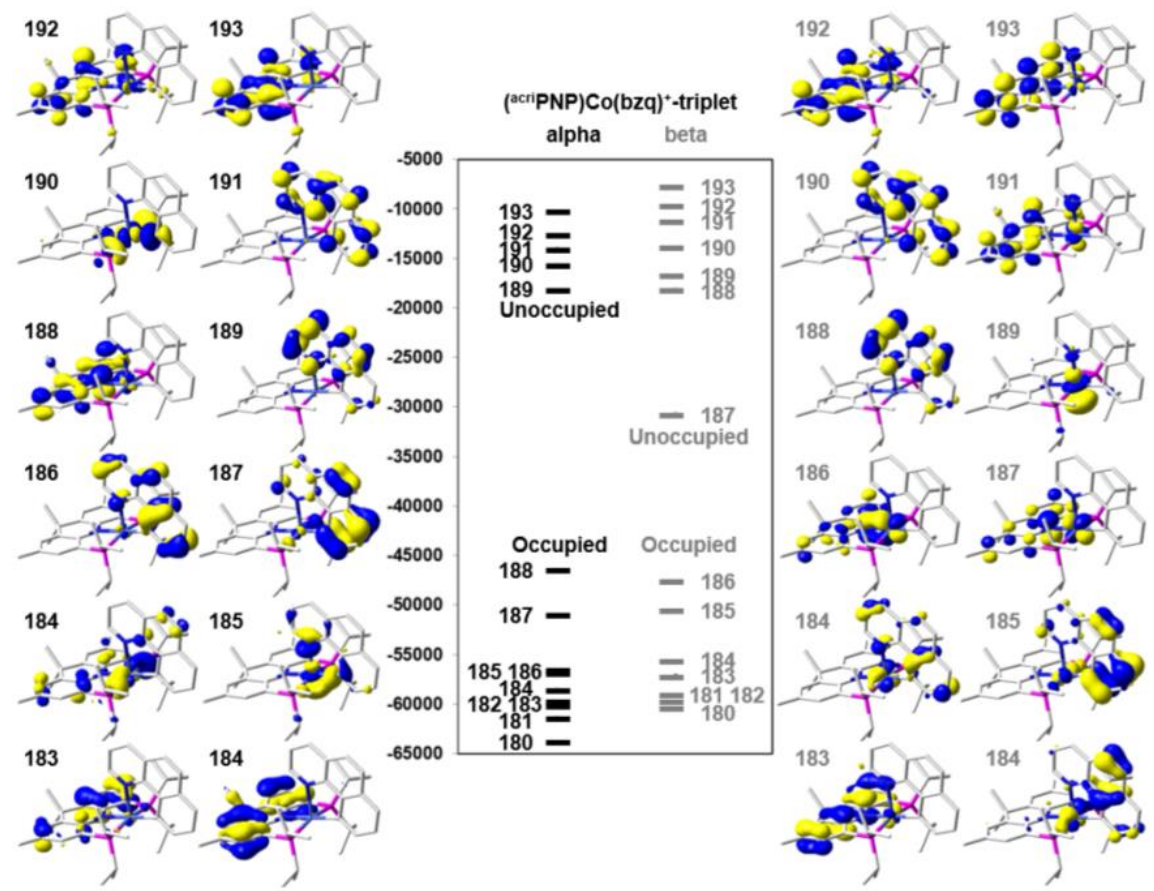


Table S15. Computed energies of $\left\{\left({ }^{\text {acriPNP }}\right) \mathrm{Co}(\mathrm{ppy})\right\}^{+}\left(\mathbf{2}-\mathbf{p p y} \mathbf{y}^{+}\right)$and $\left\{\left({ }^{\text {acripNP}}\right) \mathrm{Co}(\mathrm{bzq})\right\}^{+}(\mathbf{2}-$ $\mathbf{b z q}^{+}$) in $\mathrm{kcal} \cdot \mathrm{mol}^{-1}$.

\begin{tabular}{cccccccc}
\hline 2-ppy $^{+}$ & Def2-SVP & Def2-TZVPP & $\Delta \mathrm{E}$ & $\Delta \mathrm{Z}_{0}$ & $\Delta \mathrm{E}_{\text {thermal }}$ & $-\mathrm{T} \Delta \mathrm{S}$ & $\Delta \mathrm{G}$ \\
OSS & 0 & 0 & 0 & 0 & 0 & 0 & 0 \\
CSS & 2.79 & 0.77 & 3.56 & 1.16 & -0.37 & 1.70 & 6.05 \\
Triplet & -1.51 & 0.18 & -1.33 & 0.27 & -0.05 & -0.55 & -1.67 \\
\hline 2-bzq $^{+}$ & Def2-SVP & Def2-TZVPP & $\Delta \mathrm{E}$ & $\Delta \mathrm{Z}_{0}$ & $\Delta \mathrm{E}_{\text {thermal }}$ & $-\mathrm{T} \Delta \mathrm{S}$ & $\Delta \mathrm{G}$ \\
OSS & 0 & 0 & 0 & 0 & 0 & 0 & 0 \\
CSS & 4.38 & 0.82 & 5.21 & 1.35 & -0.46 & 2.42 & 8.52 \\
Triplet & -1.69 & 0.23 & -1.46 & 0.31 & -0.05 & -0.08 & -1.29 \\
\hline
\end{tabular}

Figure S67. Electronic structures for $\{(\text { acripNP }) \operatorname{Co}(\mathrm{ppy})\}^{2+}\left(\mathbf{2}-\mathbf{p p y} \mathbf{2}^{2+}\right)$ derived from the geometry optimization and frequency calculations using unrestricted B3LYP level of DFT; energies in $\mathrm{cm}^{-1}$. Lobal representations correspond to the orbitals indicated by the number with 0.05 isocontours.
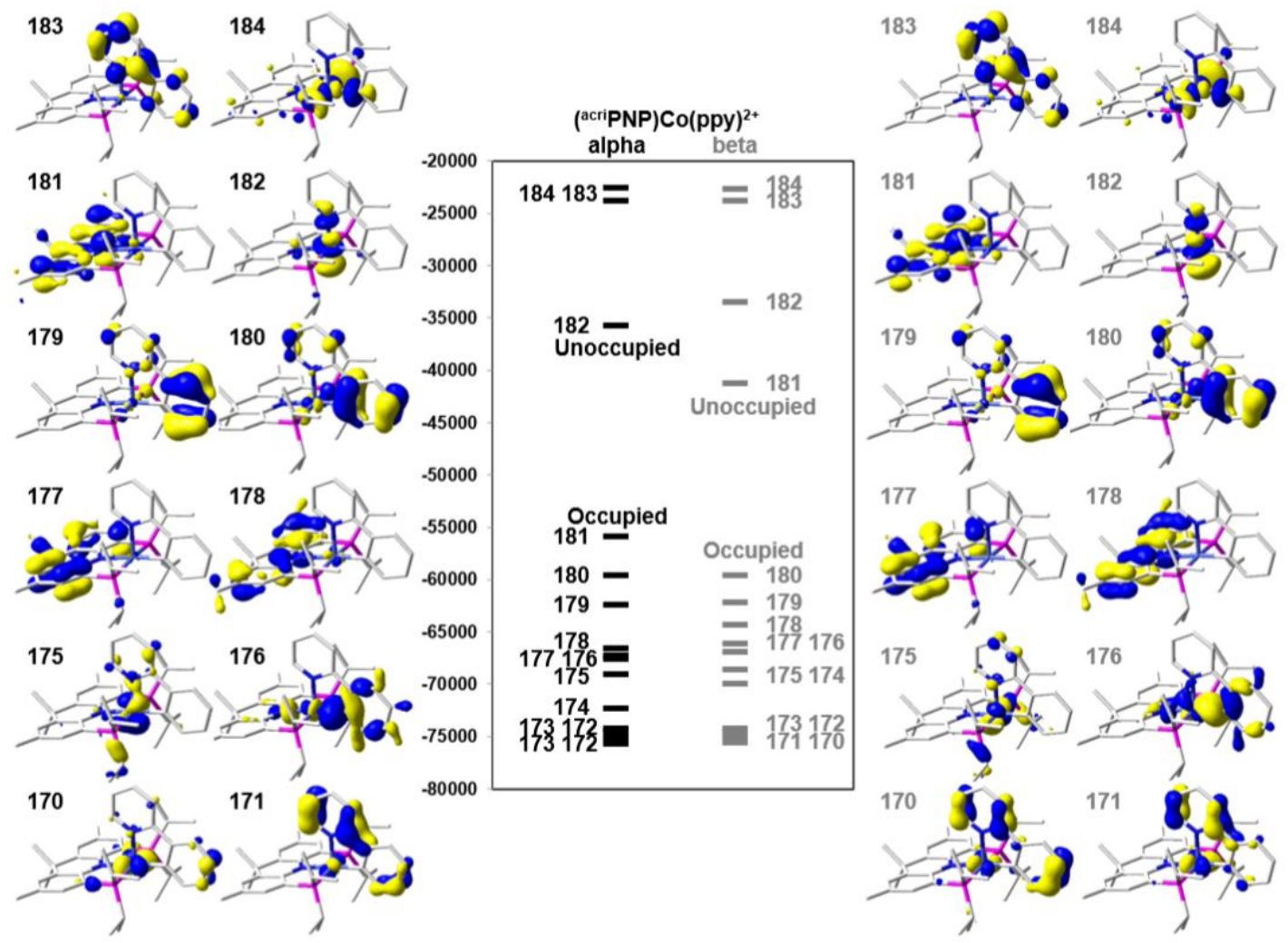
Figure S68. Electronic structures for $\left\{\left({ }^{\text {acriPNP }}\right) \operatorname{Co}(\text { bzq })\right\}^{2+}\left(\mathbf{2}-\mathbf{b z q}^{2+}\right)$ derived from the geometry optimization and frequency calculations using unrestricted B3LYP level of DFT; energies in $\mathrm{cm}^{-1}$. Lobal representations correspond to the orbitals indicated by the number with 0.05 isocontours.

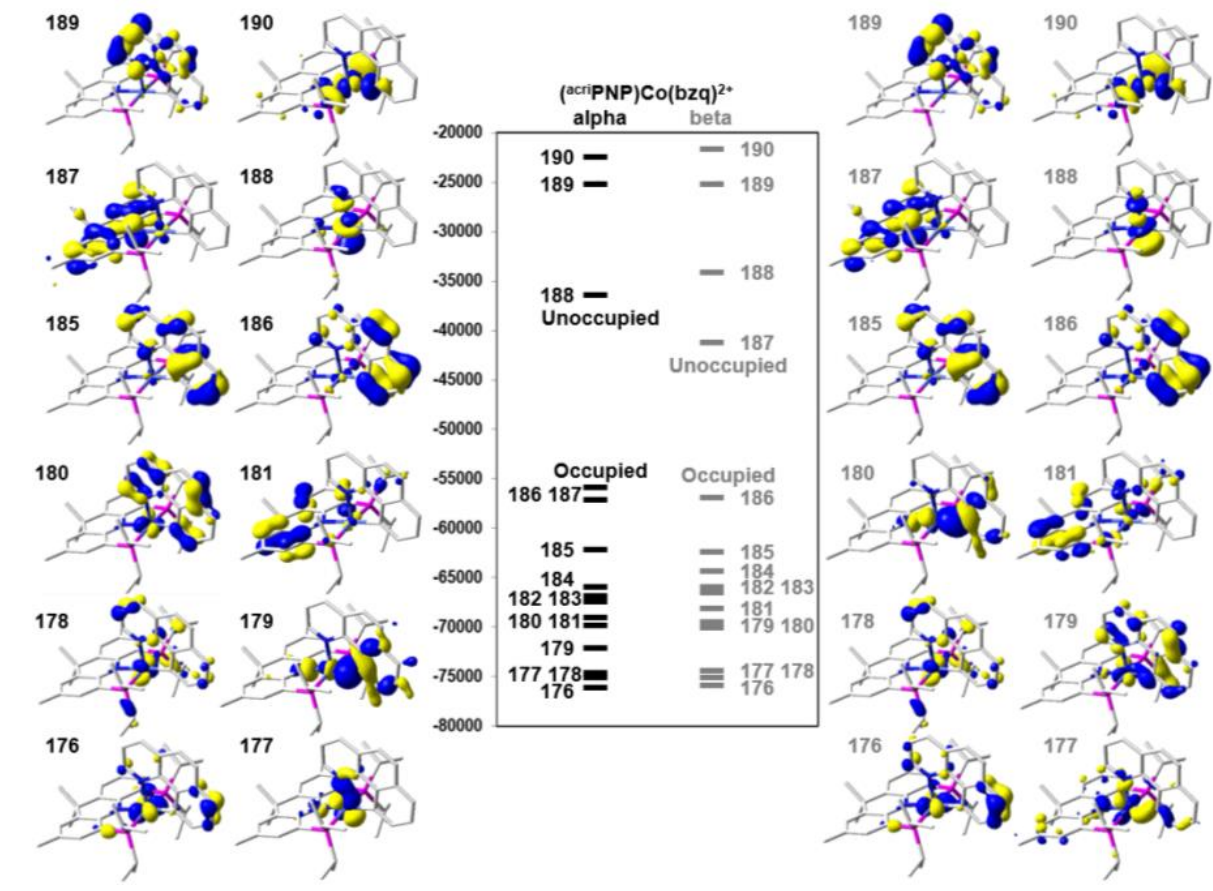

Figure S69. Electronic structures for the closed shell singlet state $\left\{\left({ }^{\text {acripNP }}\right) \operatorname{Co}(\mathrm{ppy})\right\}^{-}\left(\mathbf{2}-\mathbf{p p y} \mathbf{y}^{-}\right)$ derived from the geometry optimization and frequency calculations using unrestricted B3LYP level of DFT; energies in $\mathrm{cm}^{-1}$. Lobal representations correspond to the orbitals indicated by the number with 0.05 isocontours.

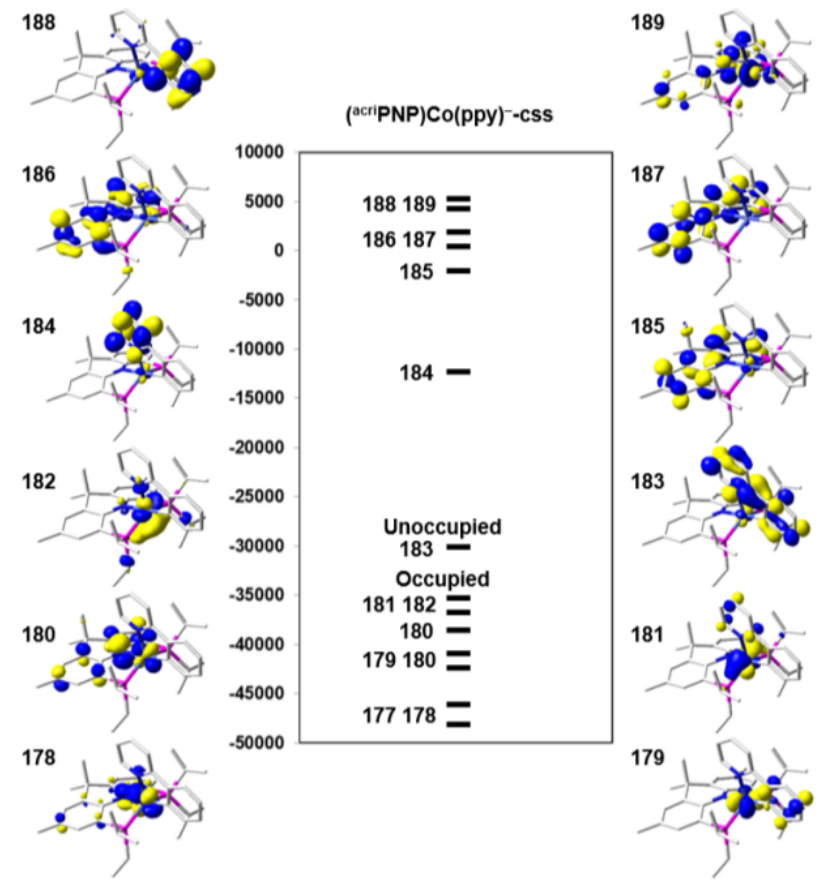




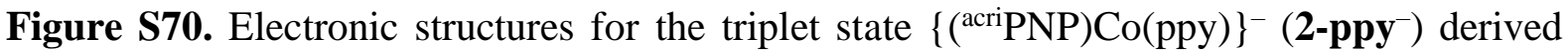
from the geometry optimization and frequency calculations using unrestricted B3LYP level of DFT; energies in $\mathrm{cm}^{-1}$. Lobal representations correspond to the orbitals indicated by the number with 0.05 isocontours.

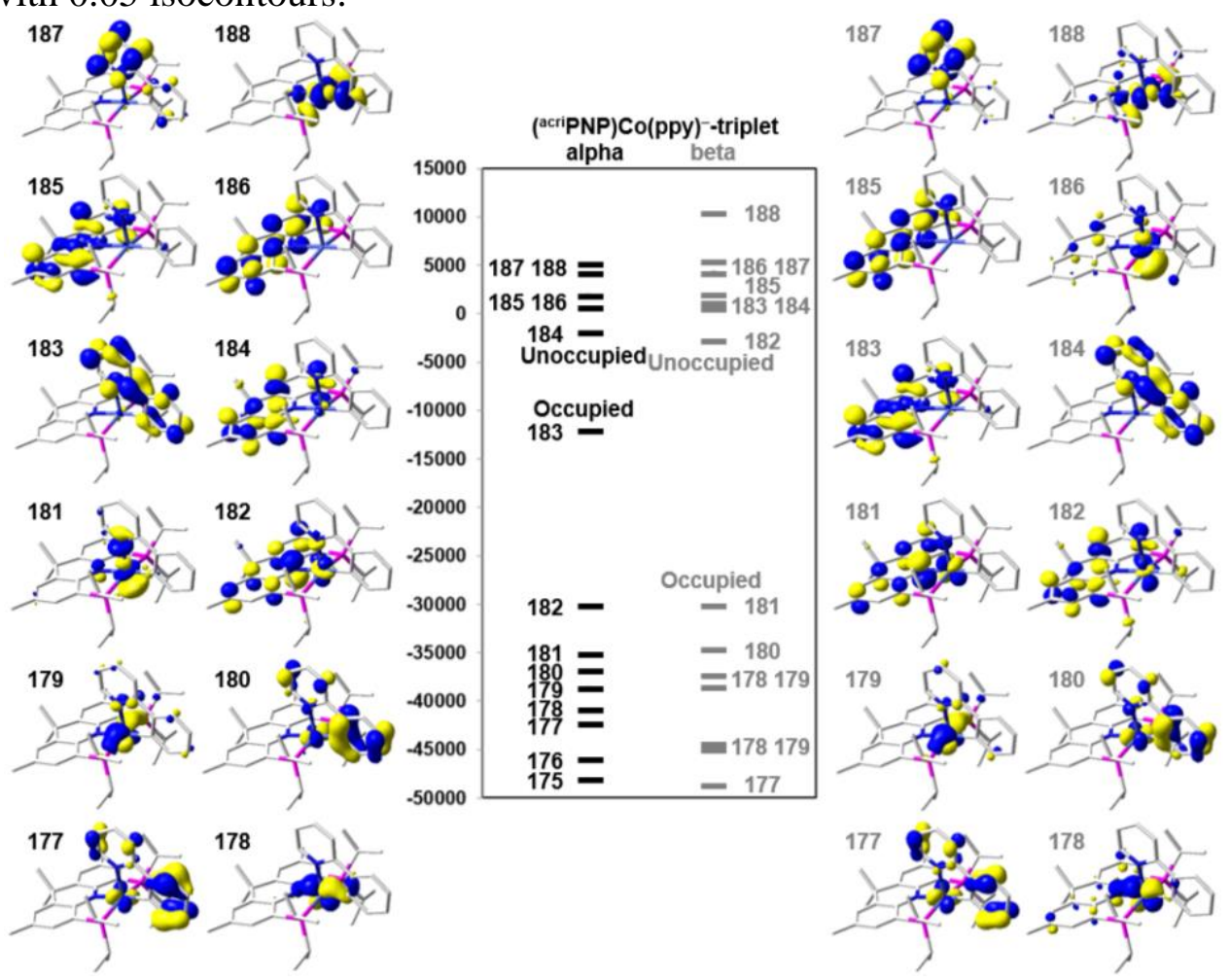

Table S16. Computed energies of $\left\{\left({ }^{\text {acripNP }}\right) \operatorname{Co}(\mathrm{ppy})\right\}^{-}\left(\mathbf{2}-\mathbf{p p y} \mathbf{y}^{-}\right)$in $\mathrm{kcal} \cdot \mathrm{mol}^{-1}$.

\begin{tabular}{cccccccc}
\hline 2-ppy & Def2-SVP & Def2-TZVPP & $\Delta \mathrm{E}$ & $\Delta \mathrm{Z}_{0}$ & $\Delta \mathrm{E}_{\text {thermal }}$ & $-\mathrm{T} \Delta \mathrm{S}$ & $\Delta \mathrm{G}$ \\
CSS & 0 & 0 & 0 & 0 & 0 & 0 & 0 \\
Triplet & 1.60 & 1.47 & 3.07 & -1.44 & 0.32 & -1.54 & 0.41 \\
\hline
\end{tabular}


Figure S71. Electronic structures for the open shell singlet state of (acriPNP)Co(bpy) (4-bpy) derived from the geometry optimization and frequency calculations using unrestricted B3LYP level of DFT; energies in $\mathrm{cm}^{-1}$. Lobal representations correspond to the orbitals indicated by the number with 0.05 isocontours.

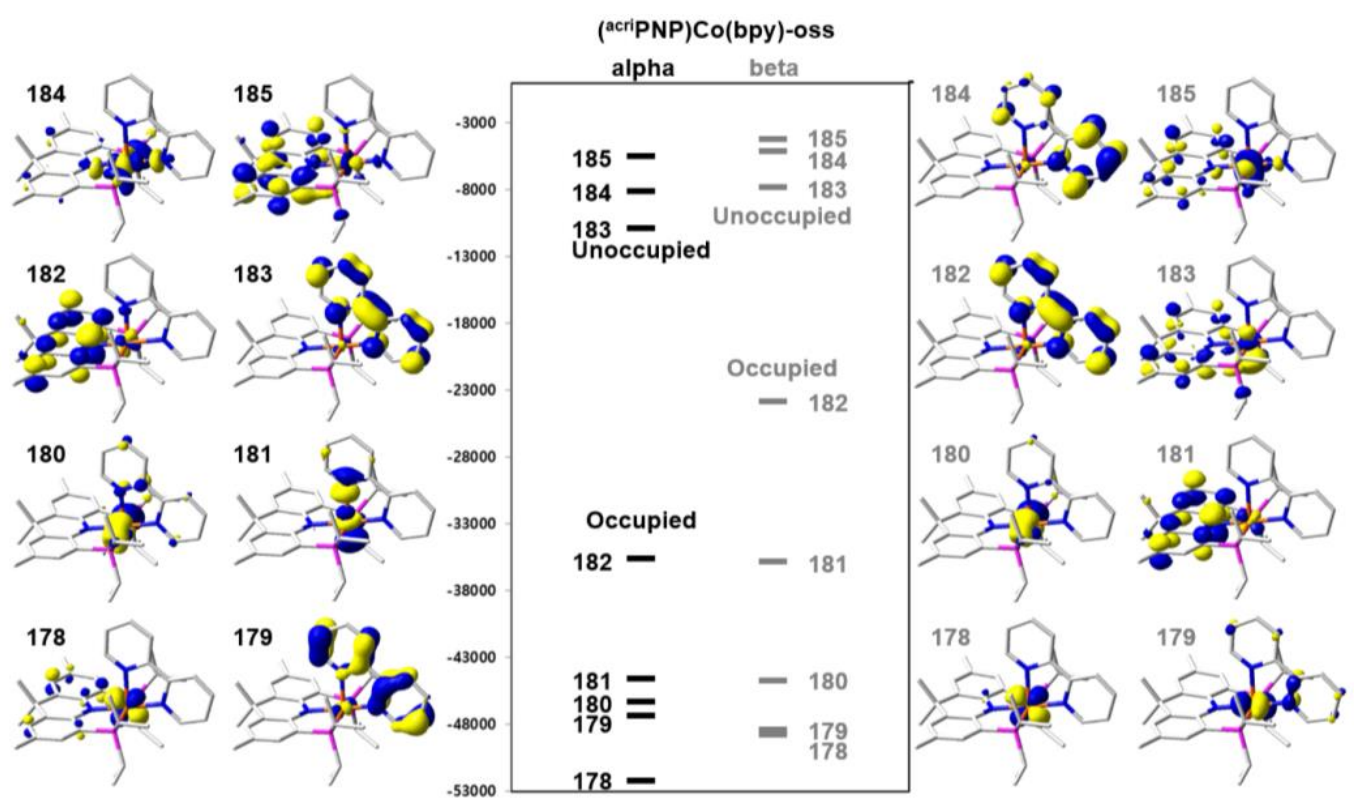

Figure S72. Electronic structures for the triplet state of (acripNP)Co(bpy) (4-bpy) derived from the geometry optimization and frequency calculations using unrestricted B3LYP level of DFT; energies in $\mathrm{cm}^{-1}$. Lobal representations correspond to the orbitals indicated by the number with 0.05 isocontours.

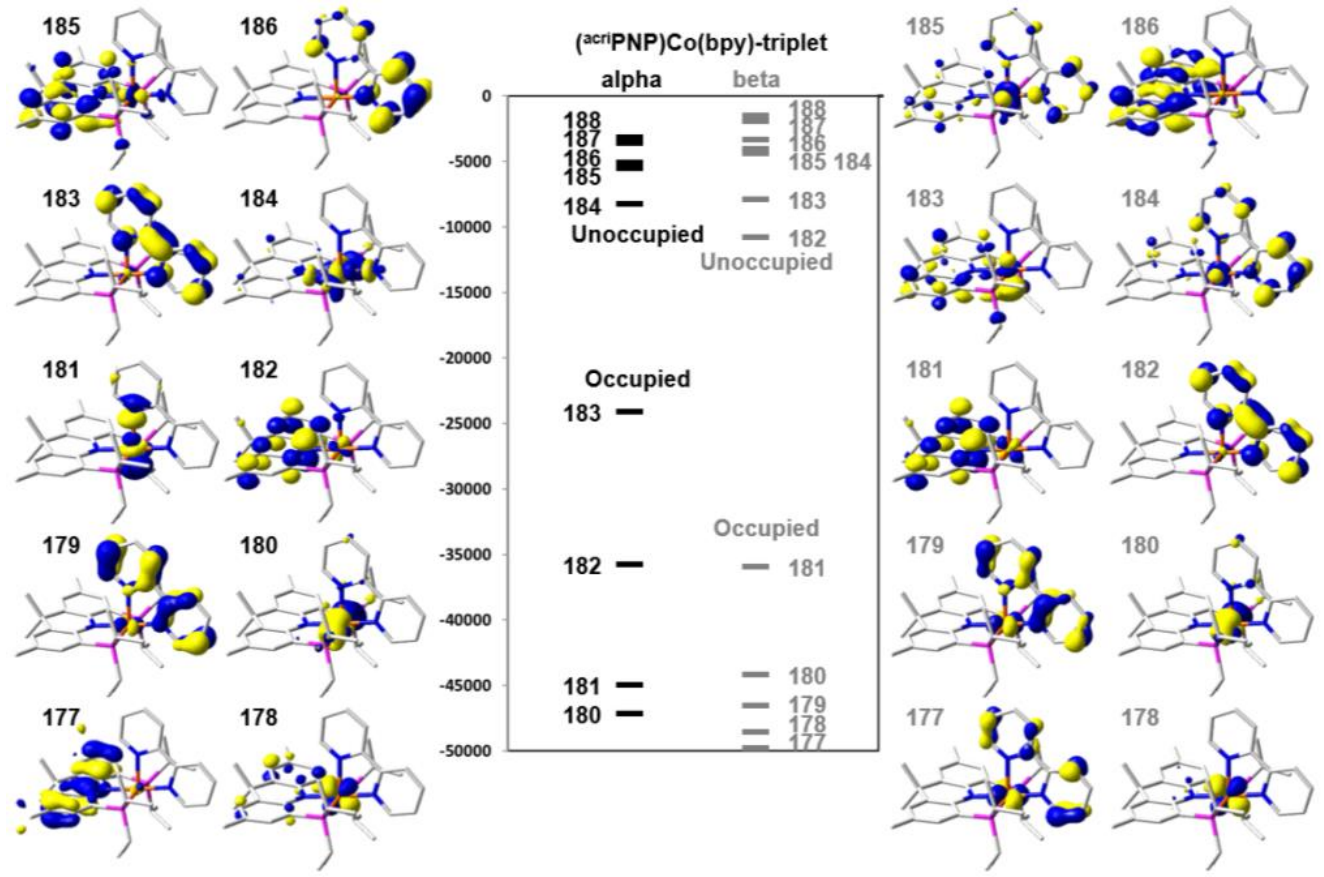


Figure S73. Electronic structures for the closed shell singlet state of (acriPNP)Co(bpy) (4-bpy) derived from the geometry optimization and frequency calculations using unrestricted B3LYP level of DFT; energies in $\mathrm{cm}^{-1}$. Lobal representations correspond to the orbitals indicated by the number with 0.05 isocontours.

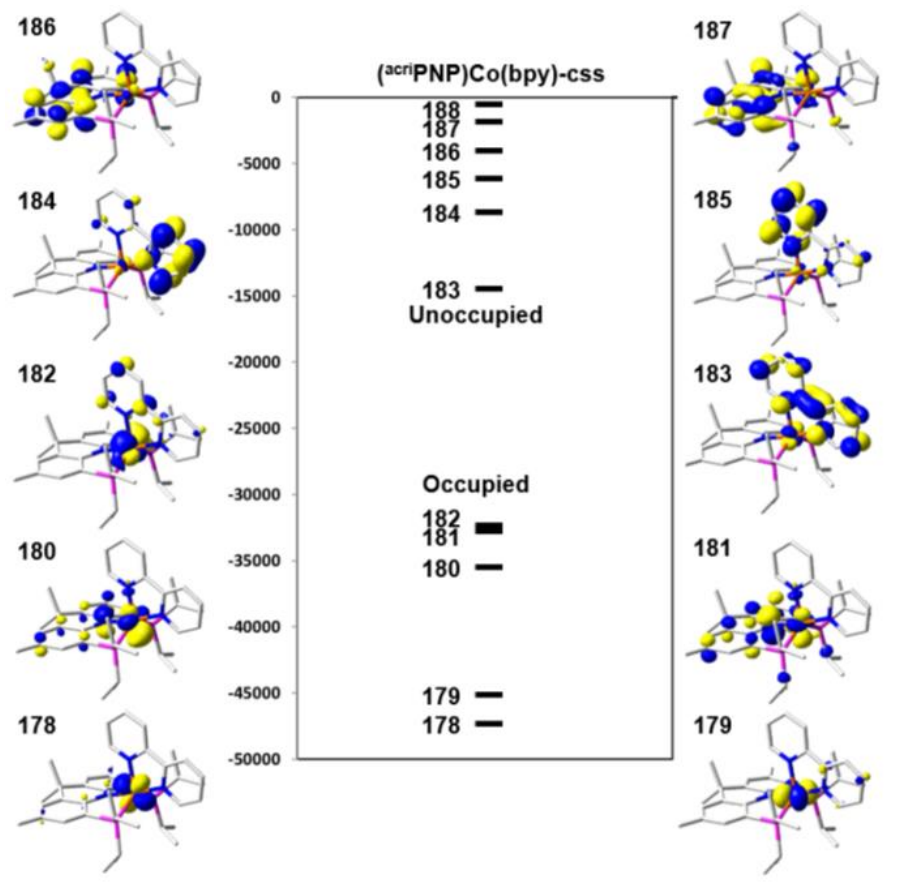

Figure S74. Electronic structures for the open shell singlet state of (acriPNP)Co(phen) (4-phen) derived from the geometry optimization and frequency calculations using unrestricted B3LYP level of DFT; energies in $\mathrm{cm}^{-1}$. Lobal representations correspond to the orbitals indicated by the number with 0.05 isocontours.

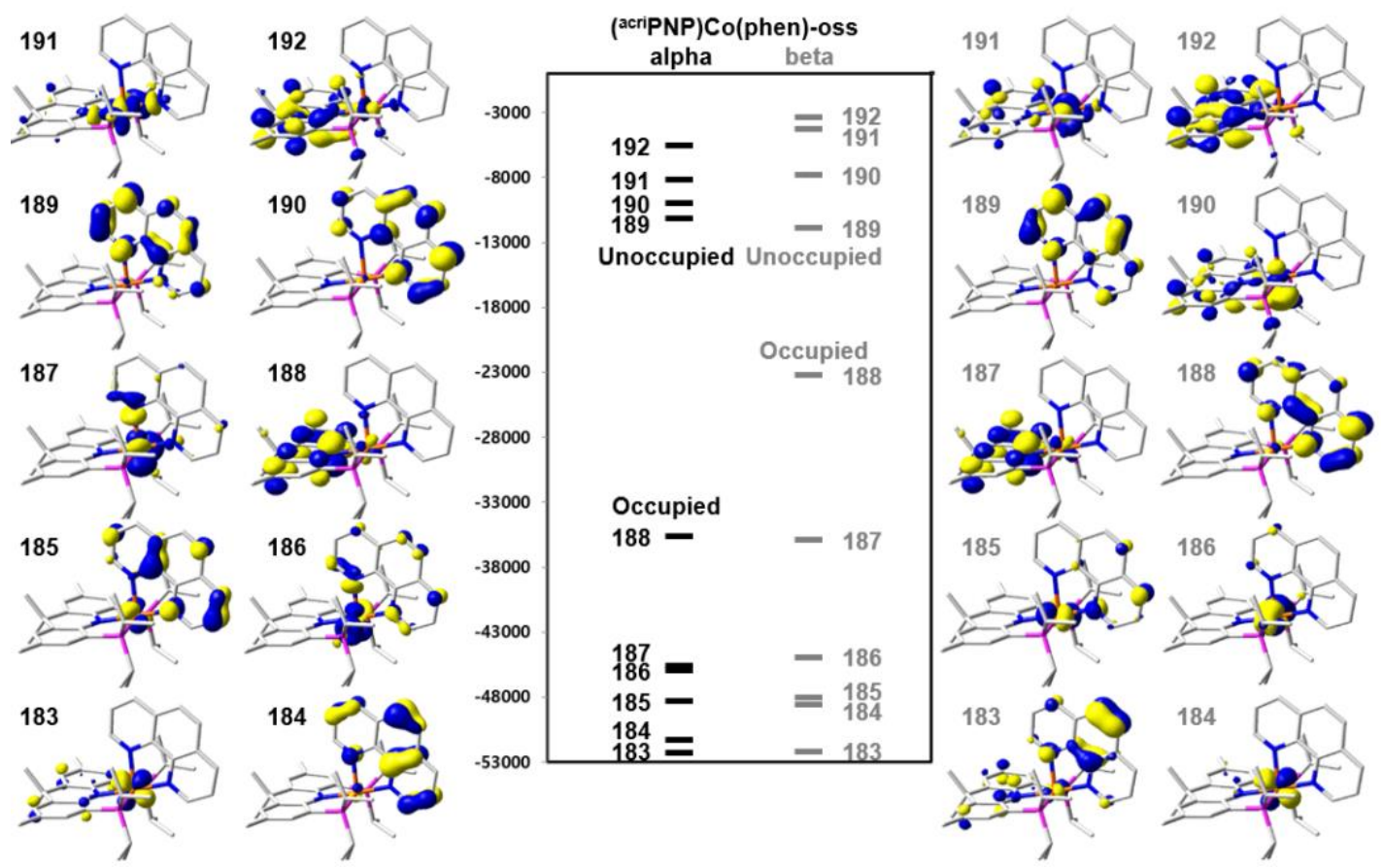


Figure S75. Electronic structures for the triplet state of (acriPNP)Co(phen) (4-phen) derived from the geometry optimization and frequency calculations using unrestricted B3LYP level of DFT; energies in $\mathrm{cm}^{-1}$. Lobal representations correspond to the orbitals indicated by the number with 0.05 isocontours.

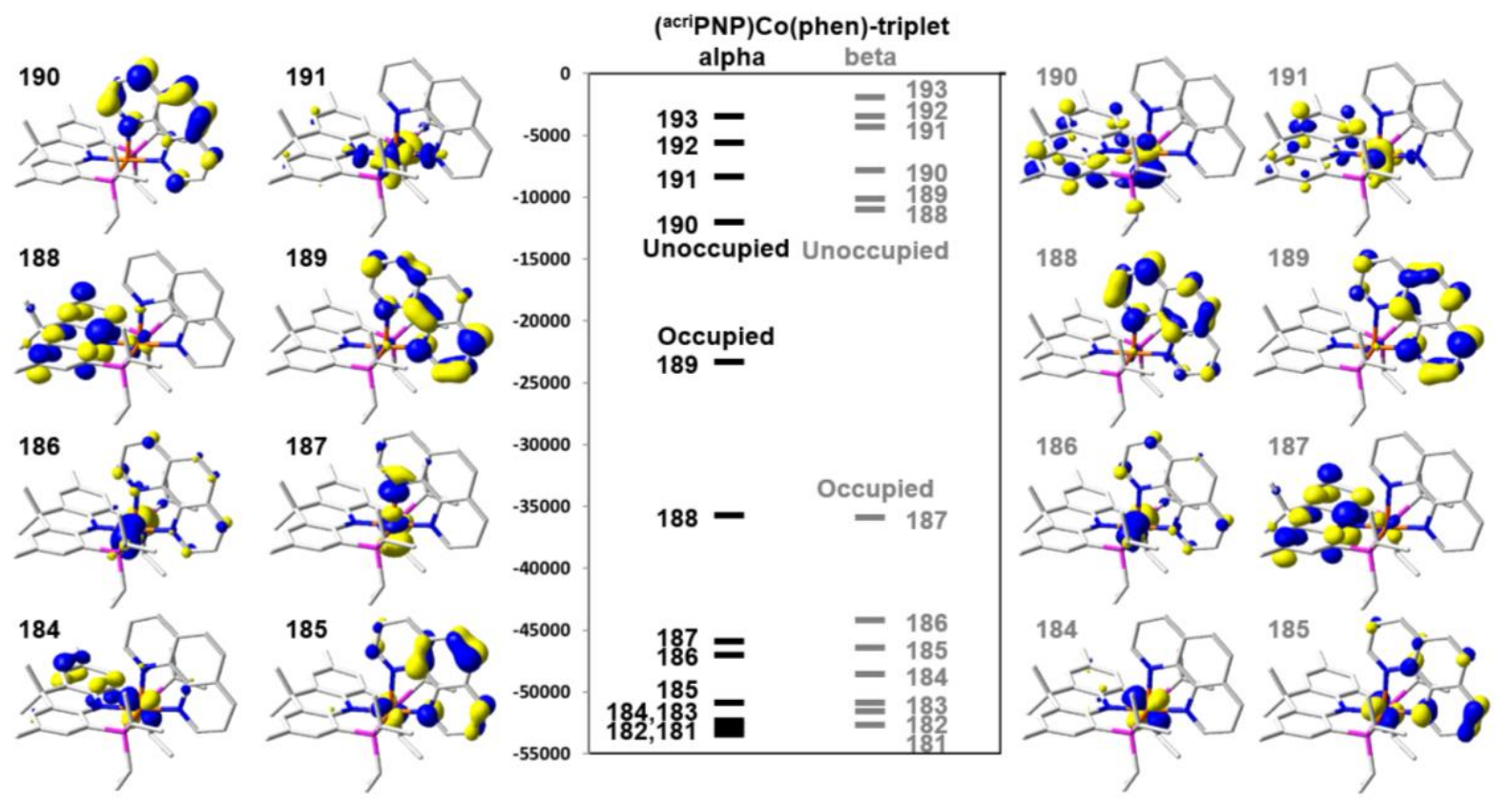

Figure S76. Electronic structures for the closed shell singlet state of (acriPNP)Co(phen) (4phen) derived from the geometry optimization and frequency calculations using unrestricted B3LYP level of DFT; energies in $\mathrm{cm}^{-1}$. Lobal representations correspond to the orbitals indicated by the number with 0.05 isocontours.

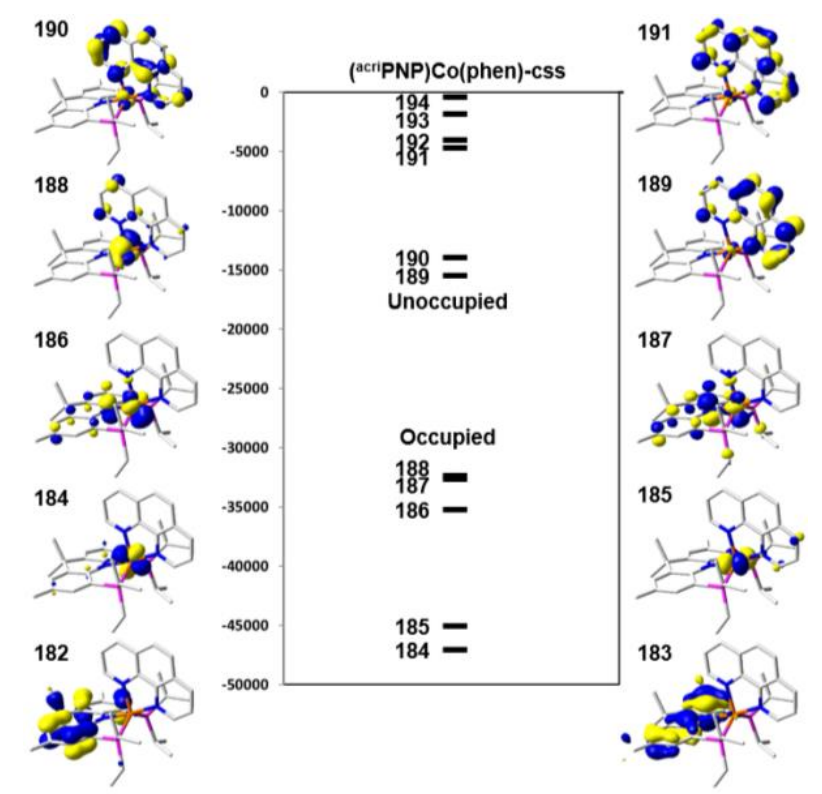


Table S17. Computed energies of (acripNP)Co(bpy) (4-bpy) and (acriPNP)Co(phen) (4-phen) in $\mathrm{kcal} \cdot \mathrm{mol}^{-1}$.

\begin{tabular}{cccccccc}
\hline 4-bpy & Def2-SVP & Def2-TZVPP & $\Delta \mathrm{E}$ & $\Delta \mathrm{Z}_{0}$ & $\Delta \mathrm{E}_{\text {thermal }}$ & $-\mathrm{T} \Delta \mathrm{S}$ & $\Delta \mathrm{G}$ \\
OSS & 0 & 0 & 0 & 0 & 0 & 0 & 0 \\
CSS & 5.05 & -1.39 & 3.67 & 0.75 & -0.19 & 0.50 & 4.73 \\
Triplet & -0.42 & -0.01 & -0.42 & 0.02 & -0.01 & -0.65 & -1.04 \\
\hline 4-phen & Def2-SVP & Def2-TZVPP & $\Delta \mathrm{E}$ & $\Delta \mathrm{Z}_{0}$ & $\Delta \mathrm{E}_{\text {thermal }}$ & $-\mathrm{T} \Delta \mathrm{S}$ & $\Delta \mathrm{G}$ \\
OSS & 0 & 0 & 0 & 0 & 0 & 0 & 0 \\
CSS & 4.78 & -1.48 & 3.30 & 1.45 & -0.22 & 0.45 & 4.97 \\
Triplet & -0.33 & -0.05 & -0.39 & 0.10 & -0.01 & -0.63 & -0.93 \\
\hline
\end{tabular}

Table S18. Mulliken spin density for the open shell singlet state of (acriPNP)Co(bpy) (4-bpy) and (acriPNP)Co(phen) (4-phen).

\begin{tabular}{ccc}
\hline & 4-bpy & 4-phen \\
\hline $\mathrm{N}_{\text {equatorial }}$ & -0.21 & -0.27 \\
$\mathrm{~N}_{\text {axial }}$ & -0.12 & -0.07 \\
$\mathrm{~N}_{\text {acriPNP }}$ & -0.02 & -0.02 \\
\hline
\end{tabular}

Figure S77. Electronic structures for $\left\{\left({ }^{\text {acri } P N P}\right) \mathrm{Co}(\mathrm{bpy})\right\}^{+}\left(\mathbf{4}-\mathbf{b p y} \mathbf{y}^{+}\right)$derived from the geometry optimization and frequency calculations using unrestricted B3LYP level of DFT; energies in $\mathrm{cm}^{-1}$. Lobal representations correspond to the orbitals indicated by the number with 0.05 isocontours.

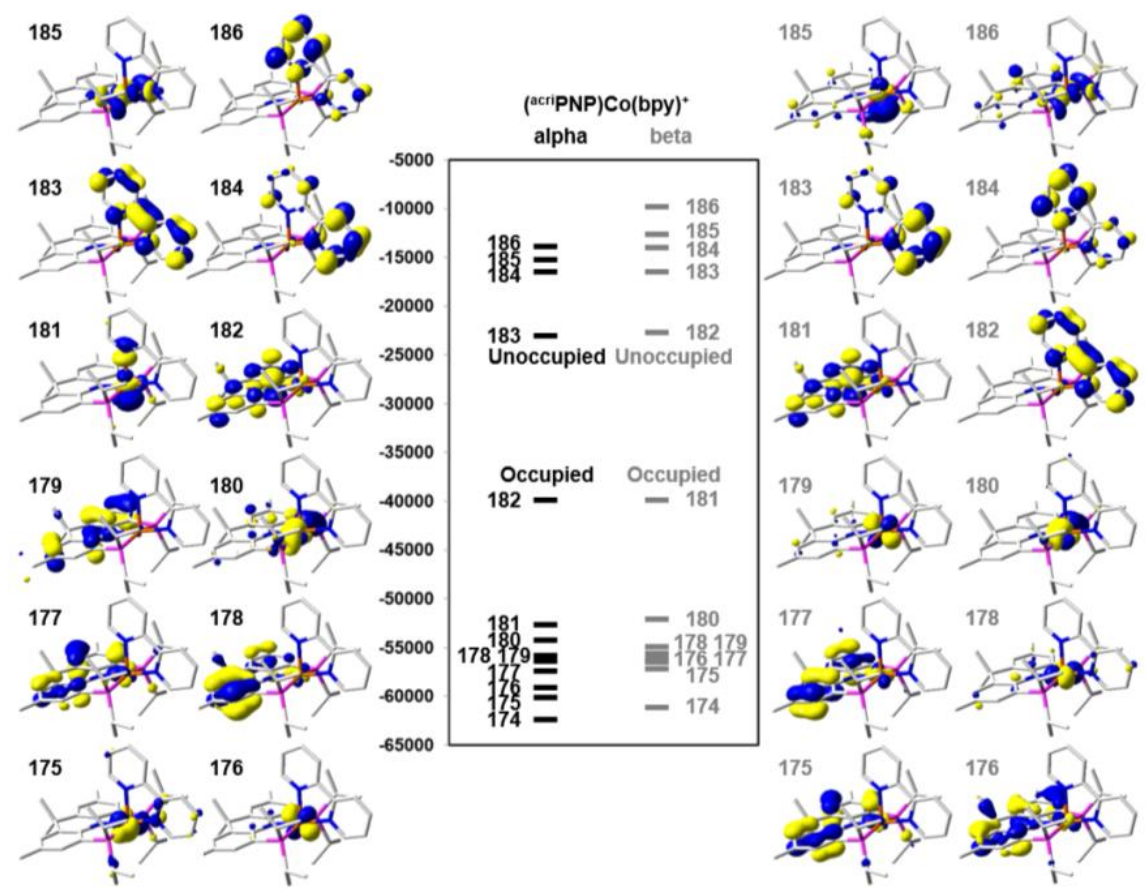


Figure S78. Electronic structures for $\left\{\left({ }^{\text {acriPNP }}\right) \operatorname{Co}(\text { phen })\right\}^{+}$(4-phen $\left.{ }^{+}\right)$derived from the geometry optimization and frequency calculations using unrestricted B3LYP level of DFT; energies in $\mathrm{cm}^{-1}$. Lobal representations correspond to the orbitals indicated by the number with 0.05 isocontours.

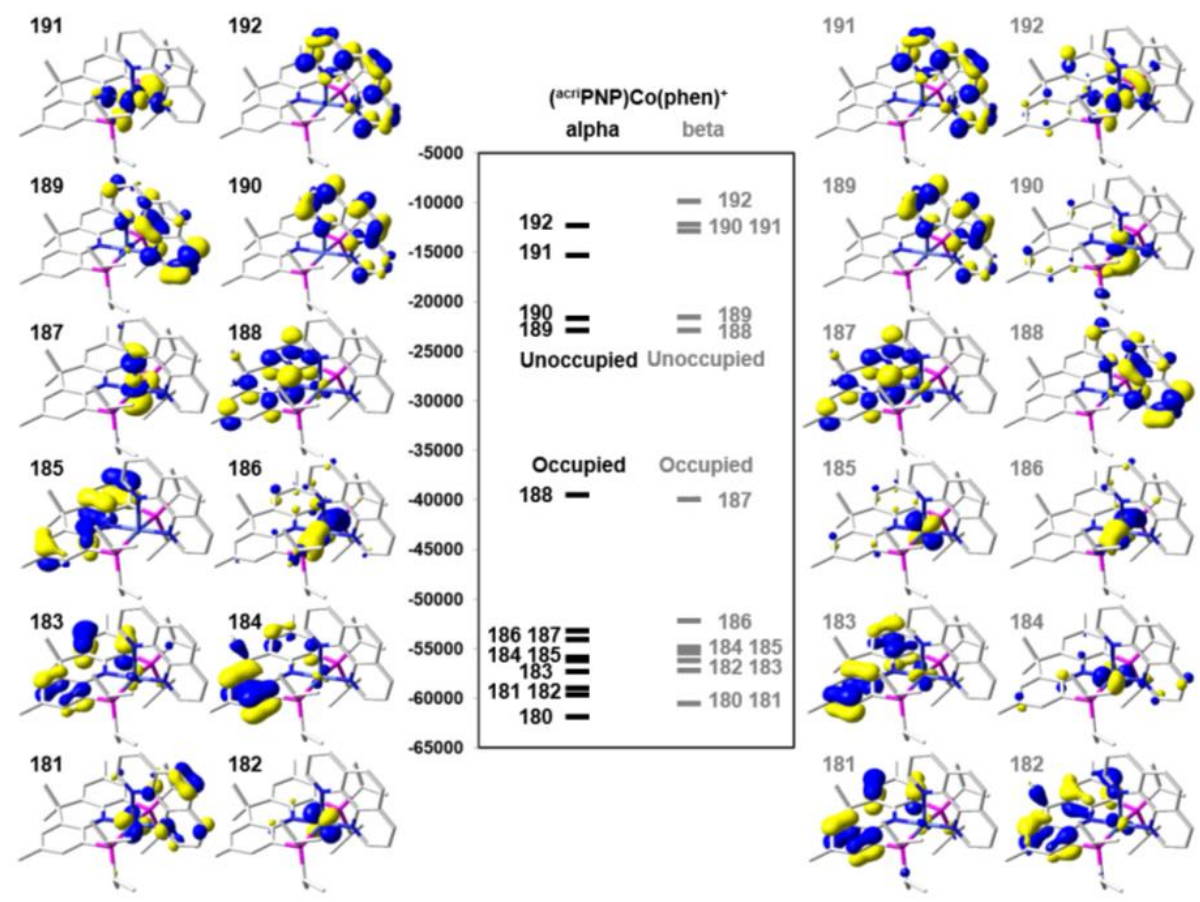

Figure S79. Electronic structures for the open-shell singlet state of 4-bpy and 4-bpy ${ }^{+}$derived from the geometry optimization and frequency calculations using unrestricted B3LYP level of DFT; energies in $\mathrm{cm}^{-1}$. Lobal representations correspond to the orbitals indicated by the number with 0.05 isocontours.

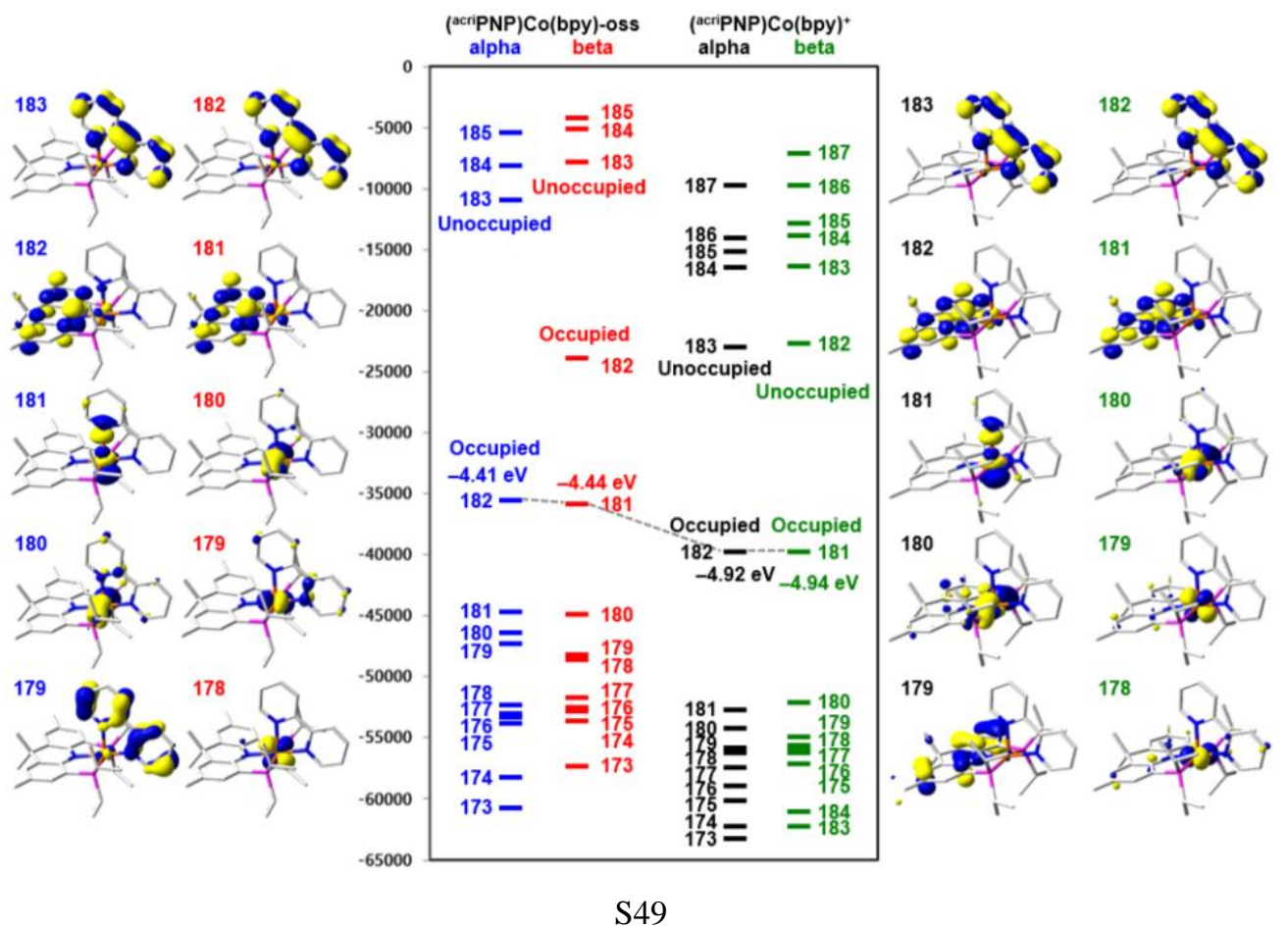


Figure S80. DFT-calculated acriPNP ligand-based orbital diagram for 4-bpy and 4-bpy ${ }^{+}$.

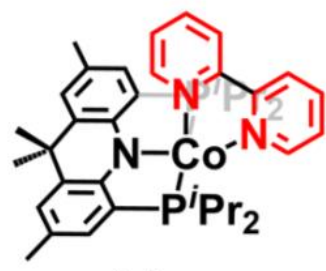

4-bpy

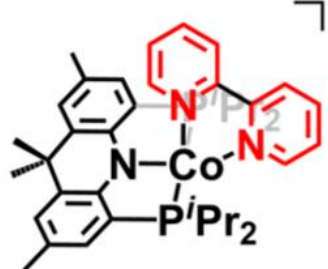

4-bpy ${ }^{+}$

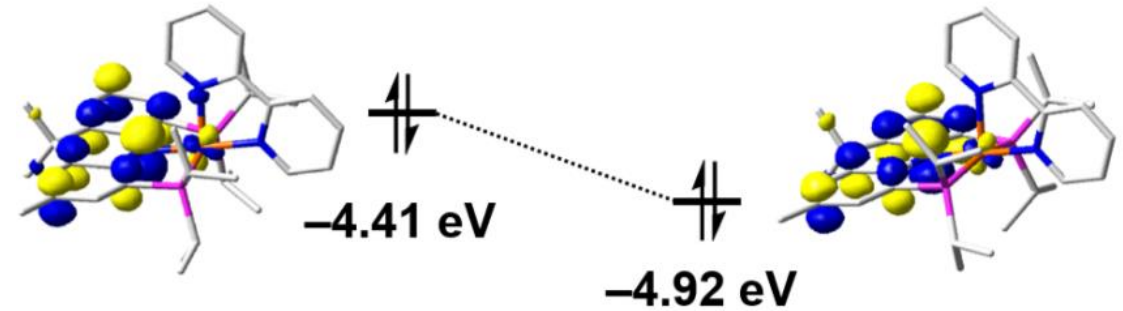

Figure S81. Electronic structures for the open shell singlet state of $\left\{\left({ }^{\text {acripNP }}\right) \operatorname{Co}(\text { bpy })\right\}^{2+}(4-$ bpy ${ }^{2+}$ ) derived from the geometry optimization and frequency calculations using unrestricted B3LYP level of DFT; energies in $\mathrm{cm}^{-1}$. Lobal representations correspond to the orbitals indicated by the number with 0.05 isocontours.
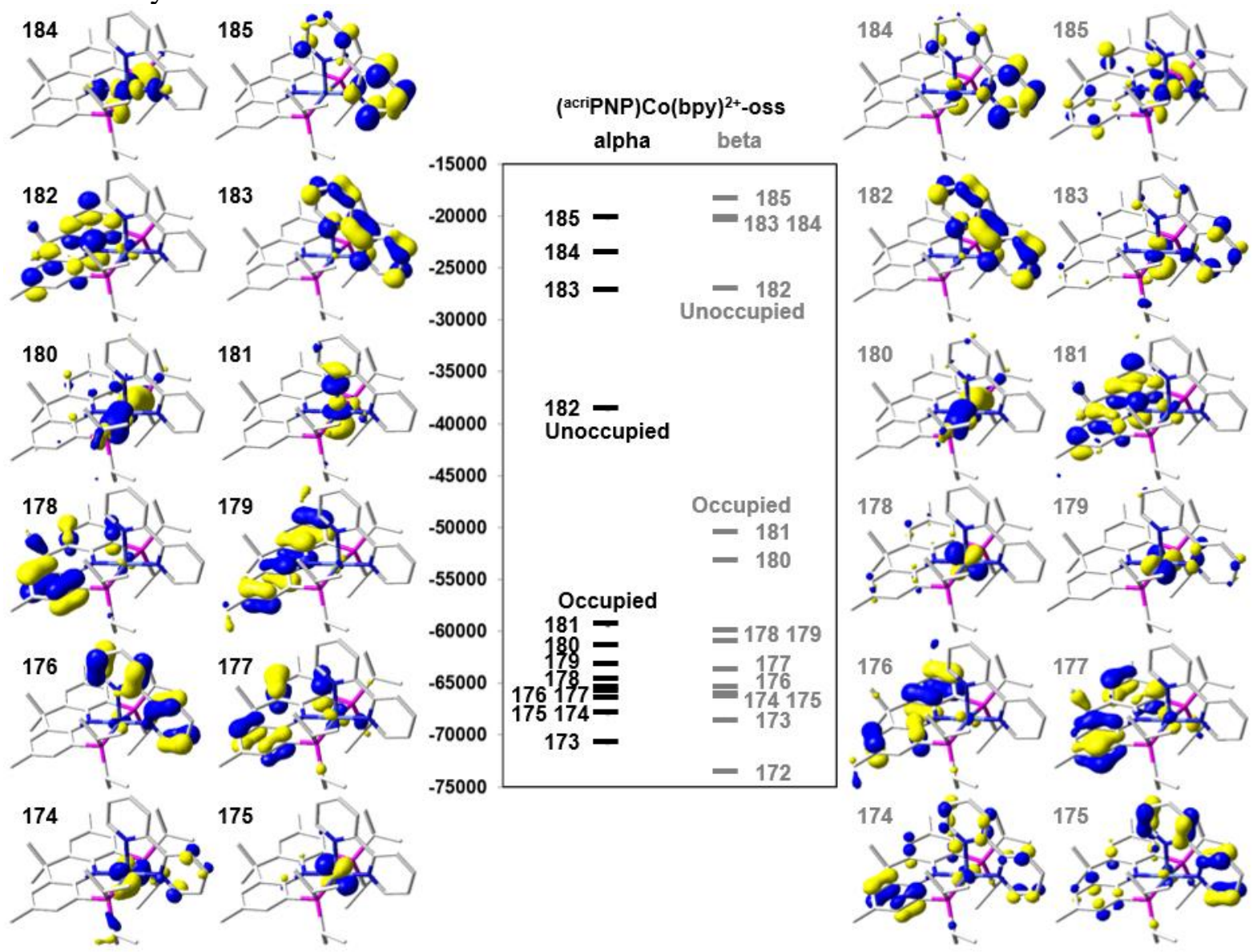


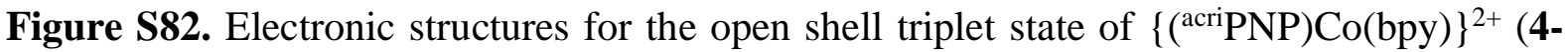
$\mathbf{b p y}^{2+}$ ) derived from the geometry optimization and frequency calculations using unrestricted B3LYP level of DFT; energies in $\mathrm{cm}^{-1}$. Lobal representations correspond to the orbitals indicated by the number with 0.05 isocontours.

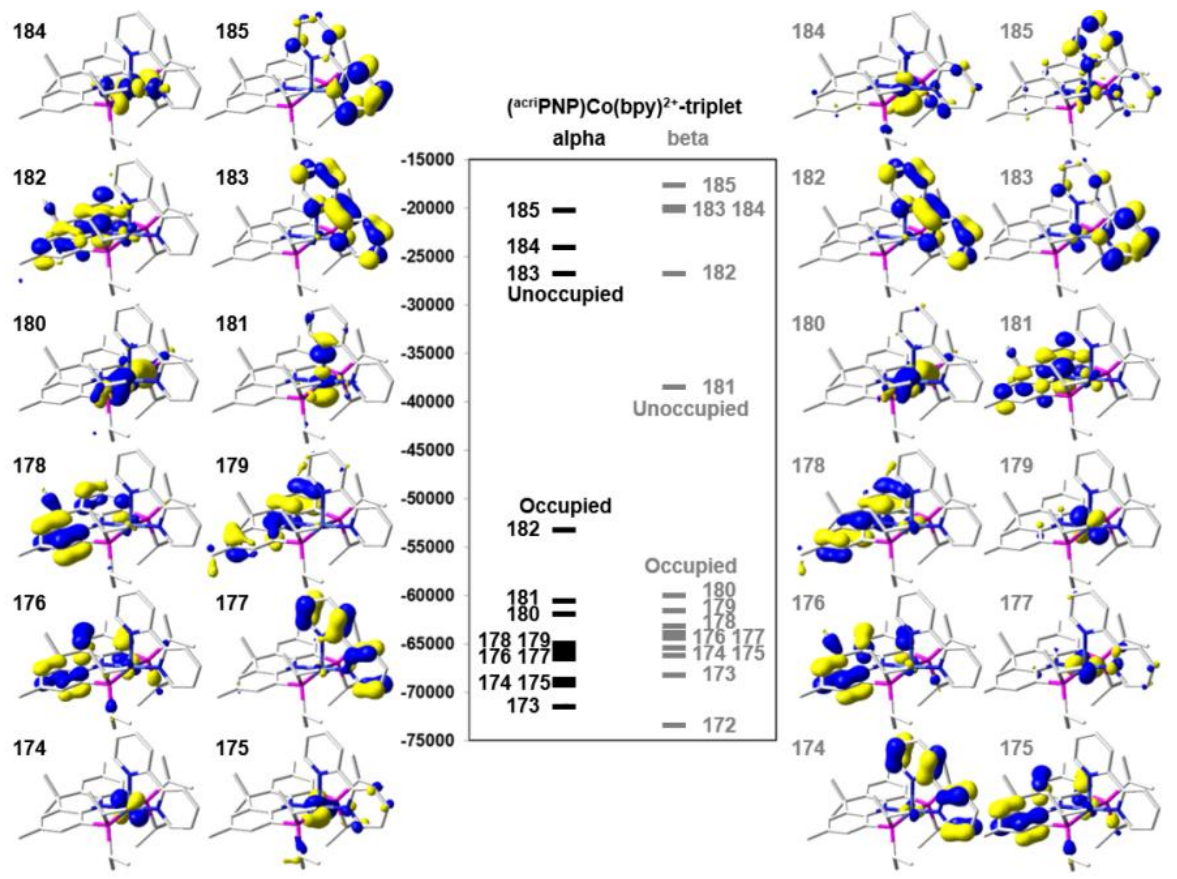

Figure S83. Electronic structures for the closed shell singlet state of $\left\{\left({ }^{\text {acripNP}}\right) \operatorname{Co}(b p y)\right\}^{2+}$ (4bpy $\left.^{2+}\right)$ and $\left\{(\text { acriPNP } \mathrm{Co}(\text { phen })\}^{2+}\left(4-\right.\right.$ phen $\left.^{2+}\right)$ derived from the geometry optimization and frequency calculations using unrestricted B3LYP level of DFT; energies in $\mathrm{cm}^{-1}$. Lobal representations correspond to the orbitals indicated by the number with 0.05 isocontours.

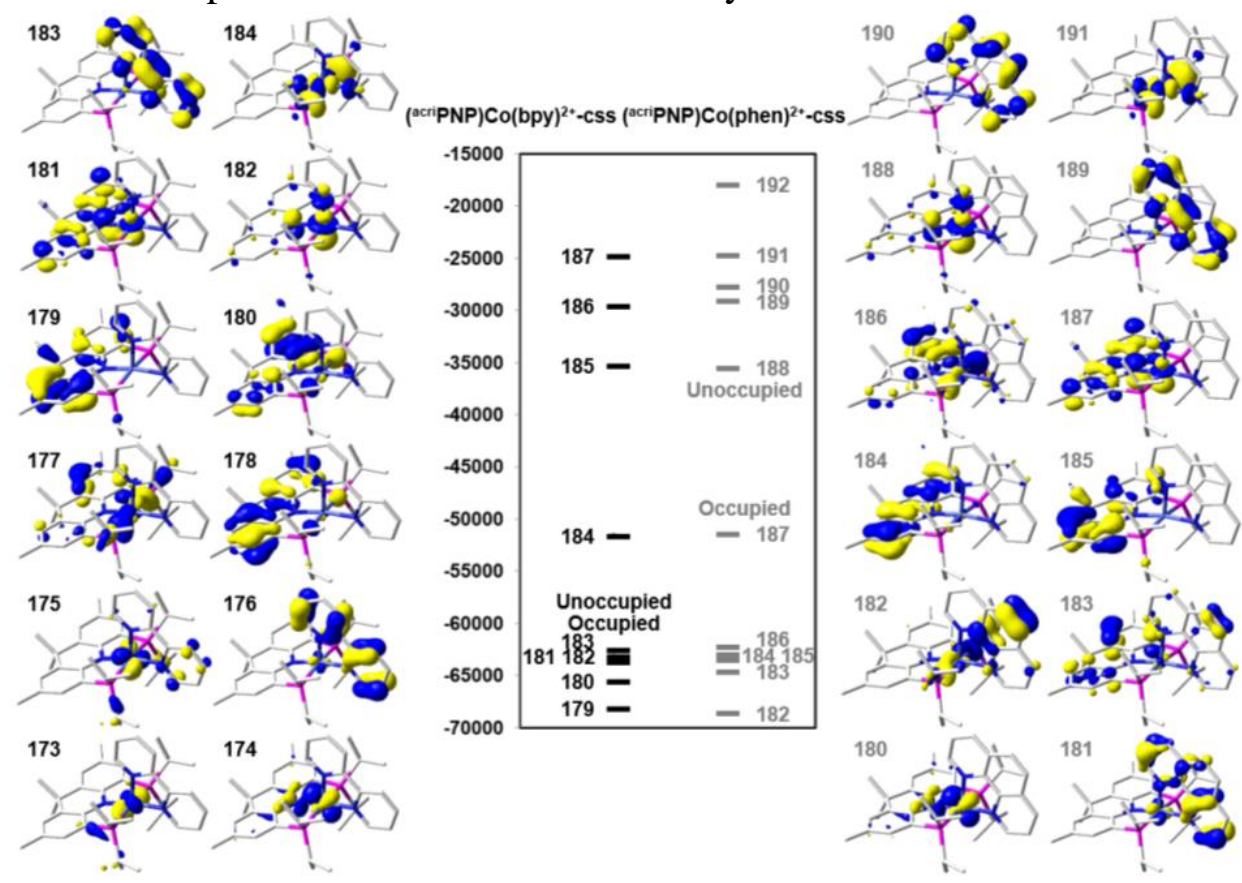


Figure S84. Electronic structures for the open shell singlet state of $\{$ (acriPNP)Co(phen $)\}^{2+}(4-$ phen $^{2+}$ ) derived from the geometry optimization and frequency calculations using unrestricted B3LYP level of DFT; energies in $\mathrm{cm}^{-1}$. Lobal representations correspond to the orbitals indicated by the number with 0.05 isocontours.

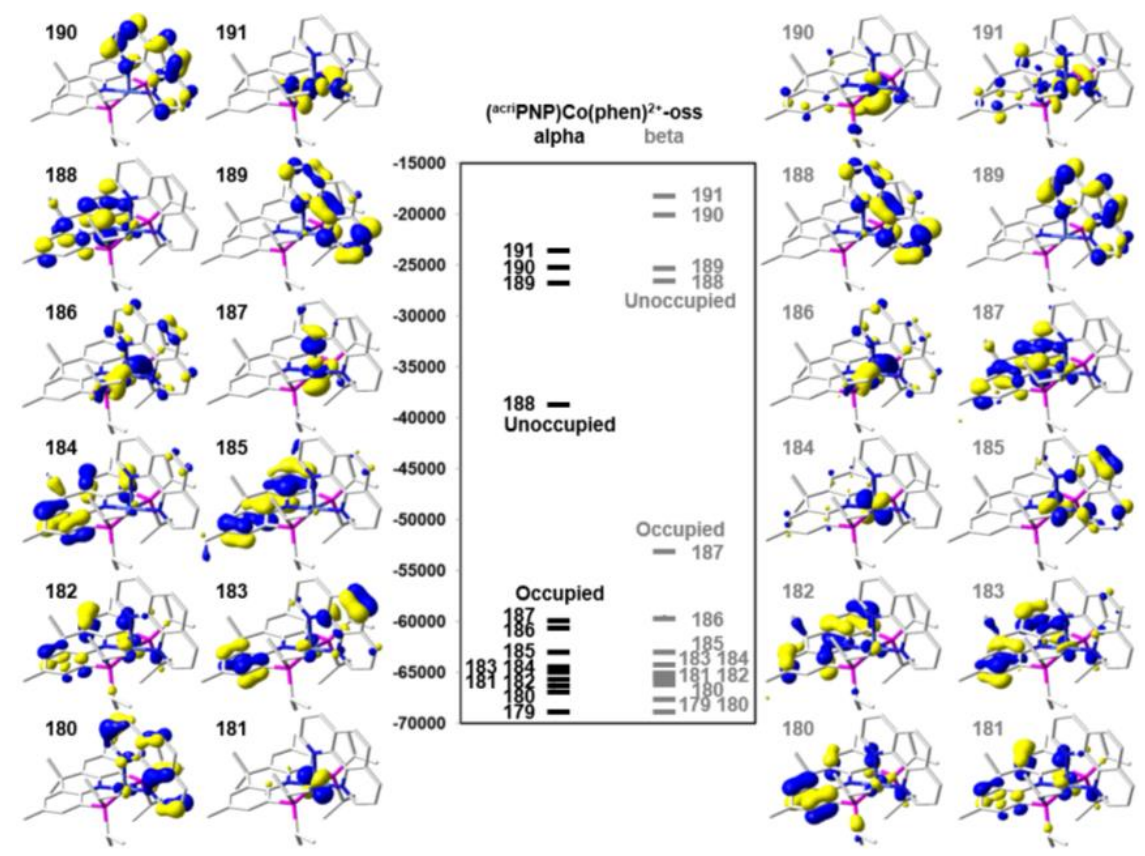

Figure S85. Electronic structures for the open shell triplet state of (acriPNP)Co(phen $)^{2+}(4-$ phen $^{2+}$ ) derived from the geometry optimization and frequency calculations using unrestricted B3LYP level of DFT; energies in $\mathrm{cm}^{-1}$. Lobal representations correspond to the orbitals indicated by the number with 0.05 isocontours.
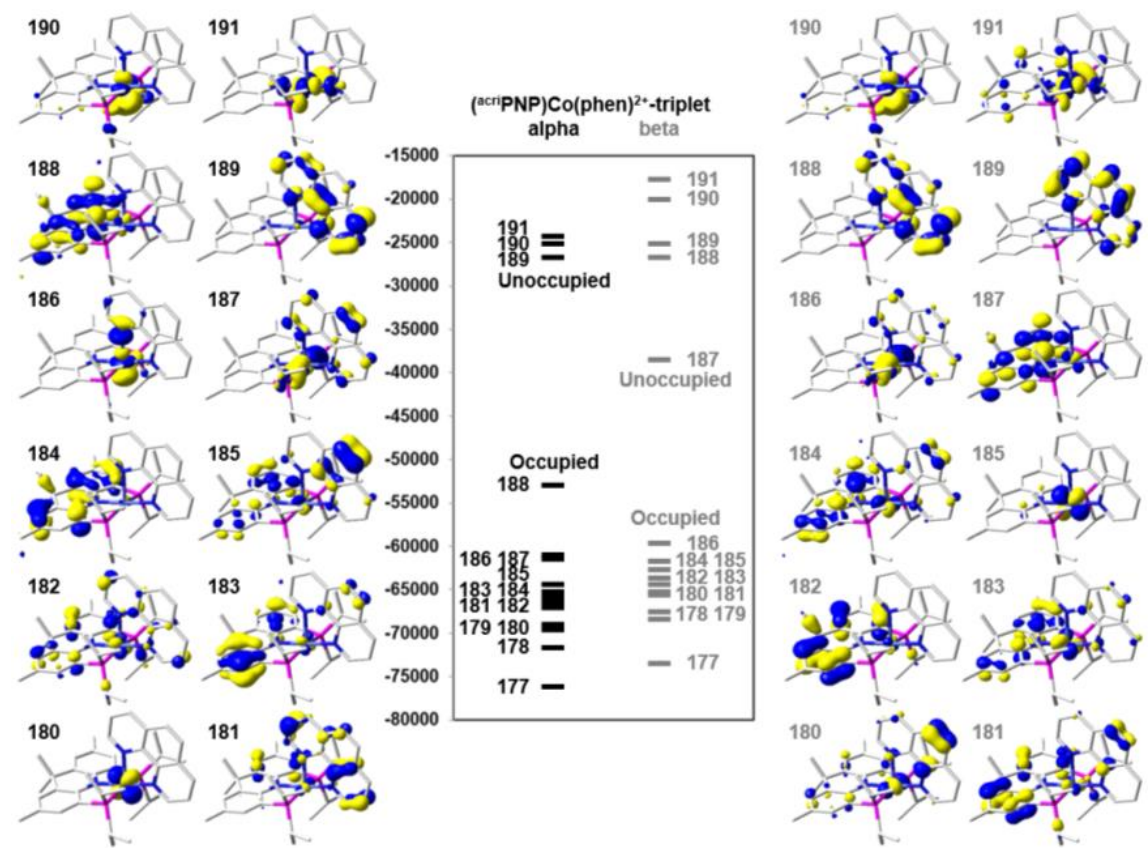
Table S19. Computed energies of $\left\{\left({ }^{\text {acripNP }}\right) \mathrm{Co}(\mathrm{bpy})\right\}^{2+}\left(\mathbf{4}-\mathrm{bpy}^{2+}\right)$ and $\left\{\right.$ ( $\left.\left.{ }^{\text {acriPNP}}\right) \mathrm{Co}(\mathrm{phen})\right\}^{2+}$ (4-phen ${ }^{2+}$ ) in $\mathrm{kcal} \cdot \mathrm{mol}^{-1}$.

\begin{tabular}{cccccccc}
\hline 4-bpy $^{2+}$ & Def2-SVP & Def2-TZVPP & $\Delta \mathrm{E}$ & $\Delta \mathrm{Z}_{0}$ & $\Delta \mathrm{E}_{\text {thermal }}$ & $-\mathrm{T} \Delta \mathrm{S}$ & $\Delta \mathrm{G}$ \\
OSS & 0 & 0 & 0 & 0 & 0 & 0 & 0 \\
CSS & 11.82 & 0.49 & 12.32 & 0.89 & -0.03 & 1.36 & 14.27 \\
Triplet & 0.11 & 0.02 & 0.13 & 0.06 & -0.29 & -0.34 & -0.18 \\
\hline 4-phen $^{2+}$ & Def2-SVP & Def2-TZVPP & $\Delta \mathrm{E}$ & $\Delta \mathrm{Z}_{0}$ & $\Delta$ Ethermal $^{-}$ & $-\mathrm{T} \Delta \mathrm{S}$ & $\Delta \mathrm{G}$ \\
OSS & 0 & 0 & 0 & 0 & 0 & 0 & 0 \\
CSS & 12.73 & 0.50 & 13.23 & 0.93 & -0.30 & 1.21 & 15.06 \\
Triplet & 0.06 & 0.01 & 0.08 & 0.05 & -0.03 & -0.35 & -0.26 \\
\hline
\end{tabular}

Figure S86. Electronic structures for the doublet of $\left\{\left({ }^{a c r i P N P}\right) \operatorname{Co}(\mathrm{bpy})\right\}^{-}\left(\mathbf{4}-\mathrm{bpy}^{-}\right)$derived from the geometry optimization and frequency calculations using unrestricted B3LYP level of DFT; energies in $\mathrm{cm}^{-1}$. Lobal representations correspond to the orbitals indicated by the number with 0.05 isocontours.

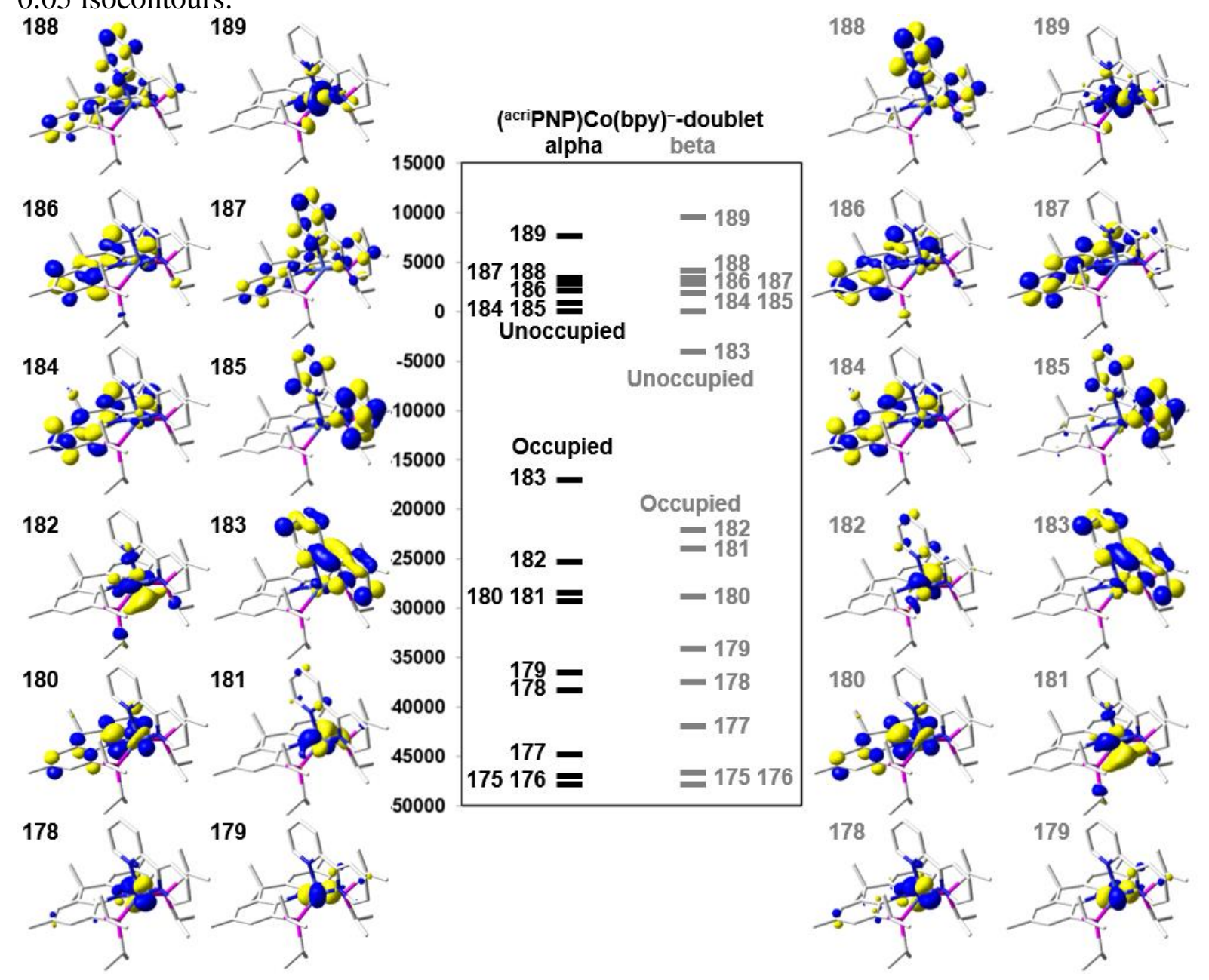


Figure S87. Electronic structures for the quartet of $\left\{\left({ }^{\text {acriPNP }}\right) \mathrm{Co}(\mathrm{bpy})\right\}^{-}\left(\right.$4-bpy $\left.{ }^{-}\right)$derived from the geometry optimization and frequency calculations using unrestricted B3LYP level of DFT; energies in $\mathrm{cm}^{-1}$. Lobal representations correspond to the orbitals indicated by the number with 0.05 isocontours.

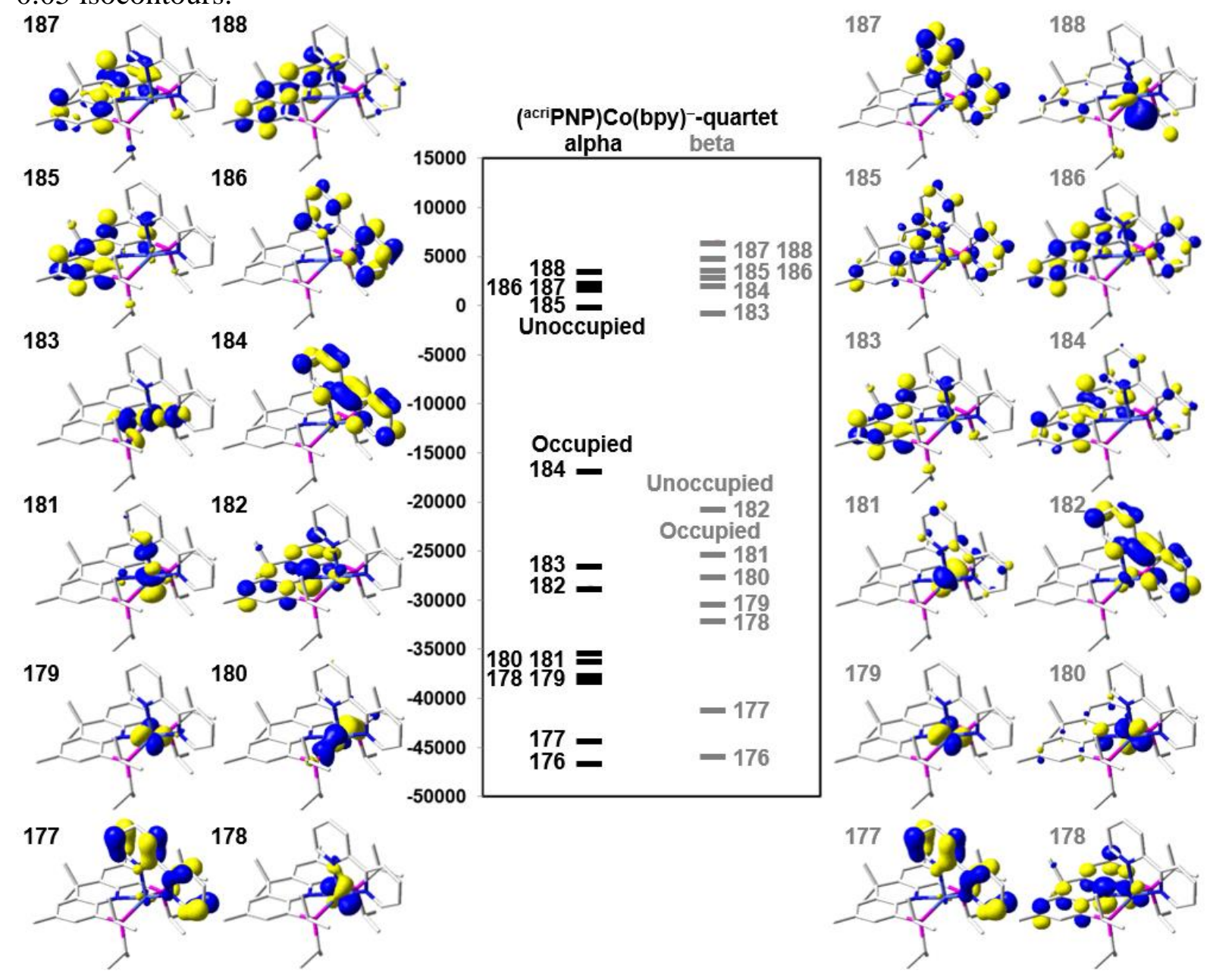

Table S20. The energy of $\left\{\left({ }^{\text {acriPNP }}\right) \mathrm{Co}(\mathrm{bpy})\right\}^{-}\left(\mathbf{4 - b p y}{ }^{-}\right)$in $\mathrm{kcal} \cdot \mathrm{mol}^{-1}$.

\begin{tabular}{cccccccc}
\hline 4-ppy $^{-}$ & Def2-SVP & Def2-TZVPP & $\Delta \mathrm{E}$ & $\Delta \mathrm{Z}_{0}$ & $\Delta \mathrm{E}_{\text {thermal }}$ & $-\mathrm{T} \Delta \mathrm{S}$ & $\Delta \mathrm{G}$ \\
Doublet & 0 & 0 & 0 & 0 & 0 & 0 & 0 \\
Quartet & -6.91 & -0.07 & -6.98 & -0.41 & 0.28 & -1.46 & -8.57 \\
\hline
\end{tabular}


Figure S88. Electronic structures for the closed shell singlet state of (acriPNP)Co(biph) (6) derived from the geometry optimization and frequency calculations using unrestricted B3LYP level of DFT; energies in $\mathrm{cm}^{-1}$. Lobal representations correspond to the orbitals indicated by the number with 0.05 isocontours.

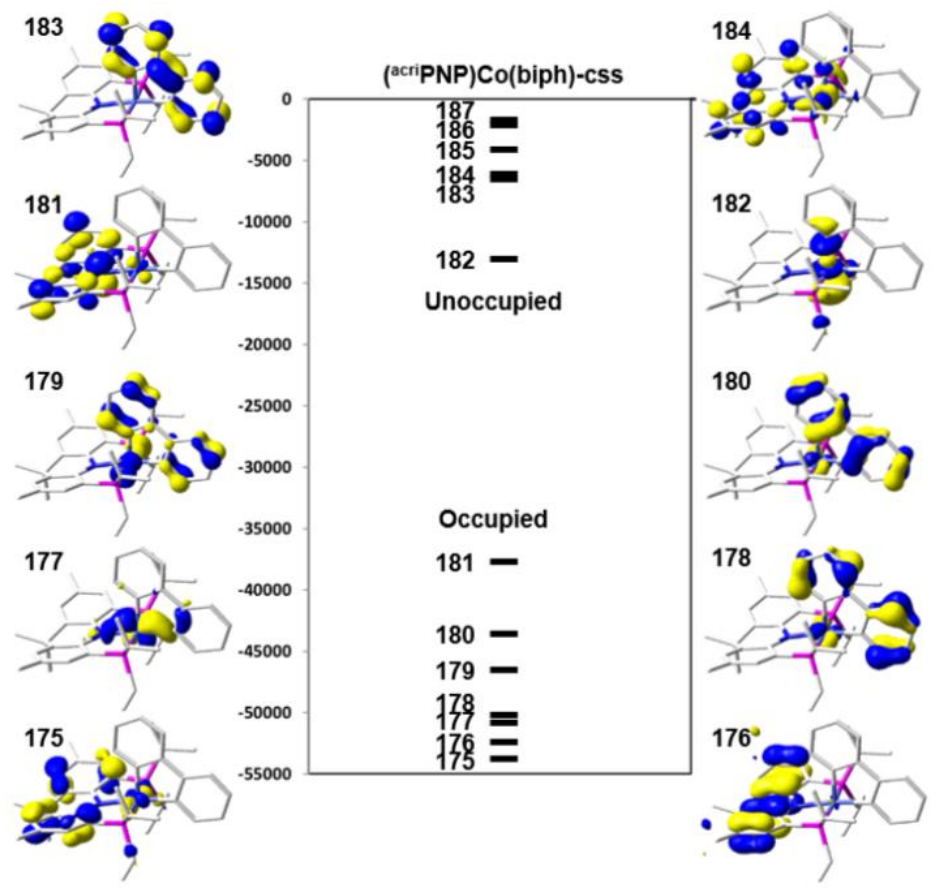

Figure S89. Electronic structures for $\left\{\left({ }^{\text {acri }} \mathrm{PNP}\right) \mathrm{Co}(\mathrm{biph})\right\}^{-}\left(\mathbf{6}^{-}\right)$derived from the geometry optimization and frequency calculations using unrestricted B3LYP level of DFT; energies in $\mathrm{cm}^{-1}$. Lobal representations correspond to the orbitals indicated by the number with 0.05 isocontours.
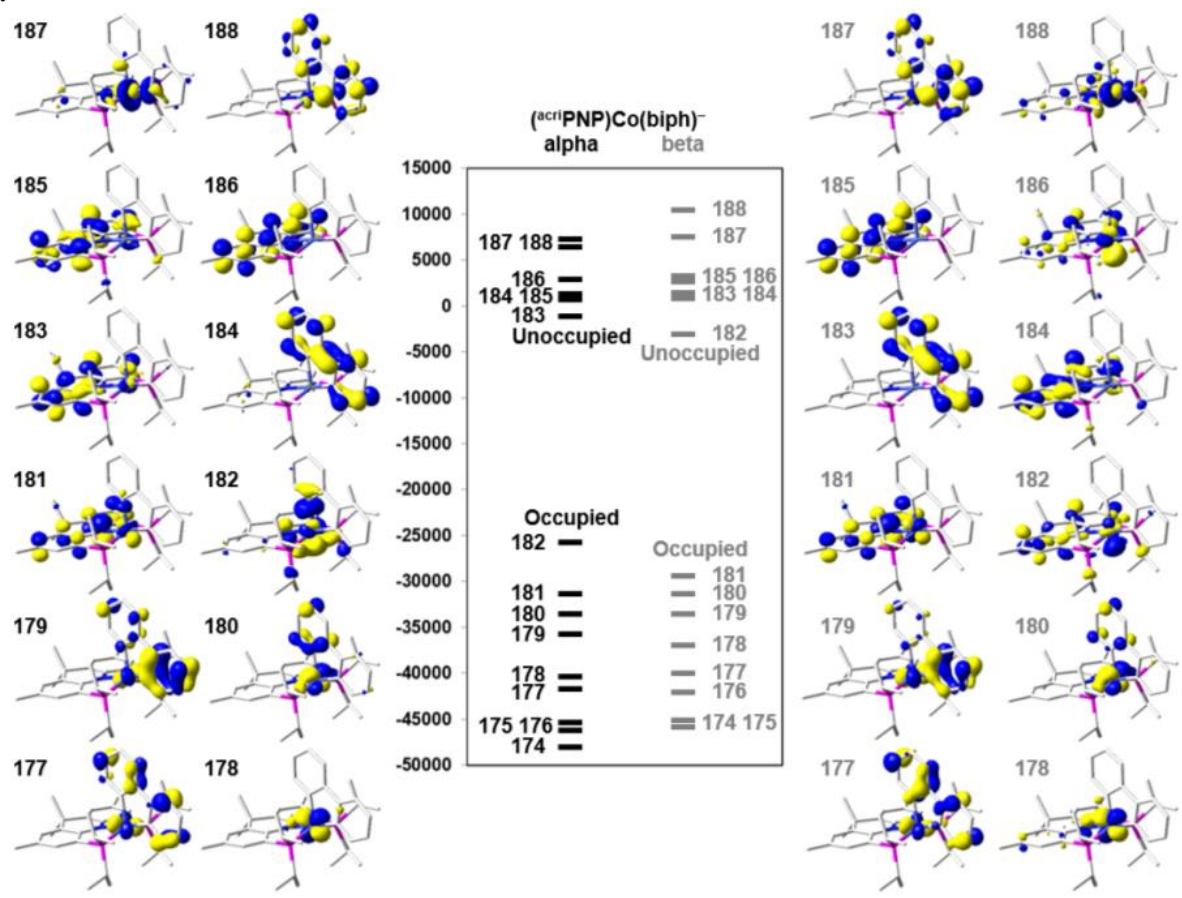
Figure S90. Electronic structures for the closed shell singlet state of $\left\{\left({ }^{\text {acripNP }}\right) \operatorname{Co}(\mathrm{biph})\right\}^{2-}$ $\left(6^{2-}\right)$ derived from the geometry optimization and frequency calculations using unrestricted B3LYP level of DFT; energies in $\mathrm{cm}^{-1}$. Lobal representations correspond to the orbitals indicated by the number with 0.05 isocontours.

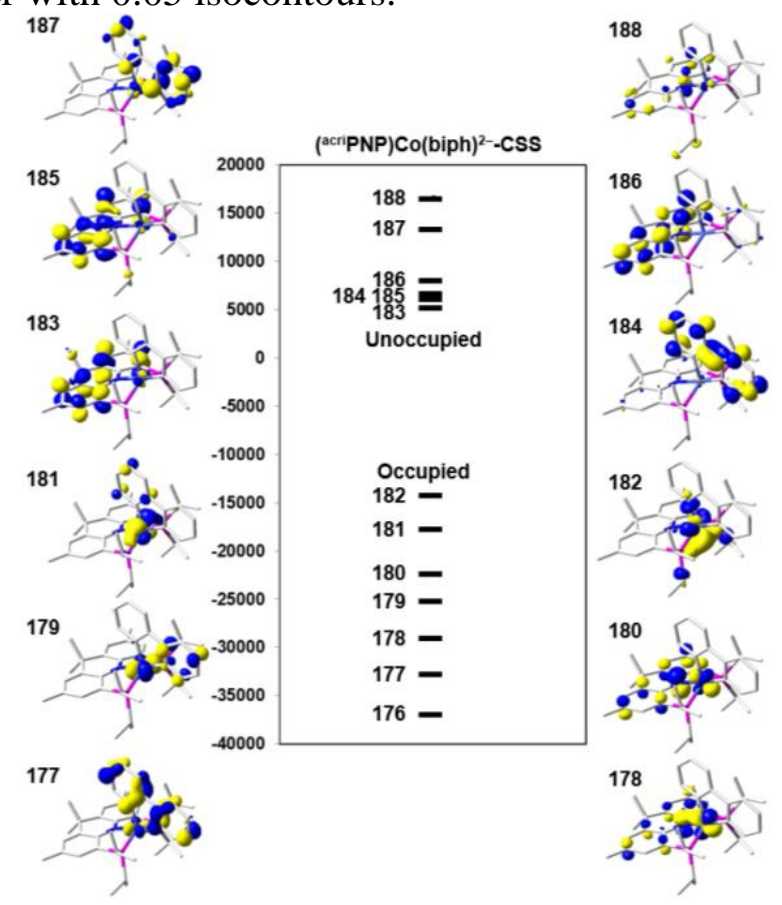

Figure S91. Electronic structures for the triplet state of $\left\{\left({ }^{\text {acriPNP }}\right) \operatorname{Co}(\text { biph })\right\}^{2-}\left(6^{2-}\right)$ derived from the geometry optimization and frequency calculations using unrestricted B3LYP level of DFT; energies in $\mathrm{cm}^{-1}$. Lobal representations correspond to the orbitals indicated by the number with 0.05 isocontours.

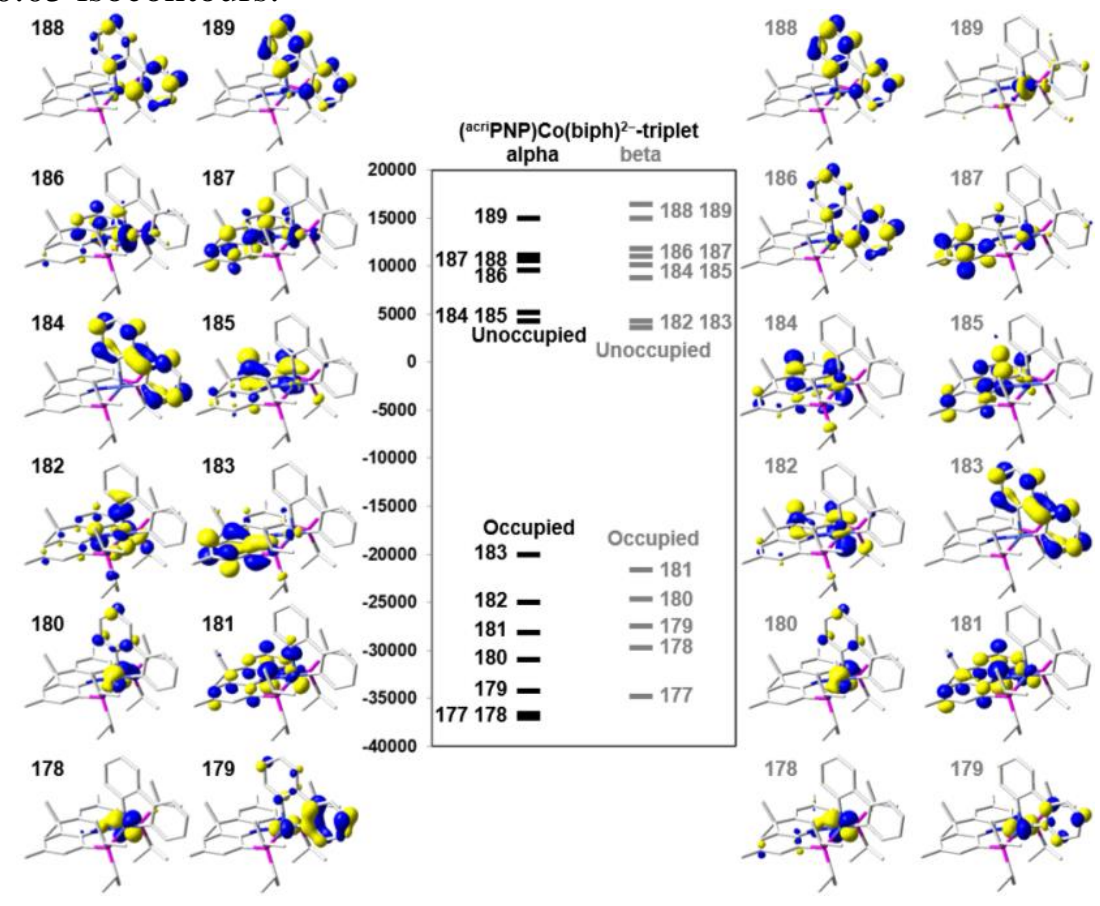


Table S21. The energy of $\left\{\left({ }^{\text {acripNP }}\right) \mathrm{Co}(\mathrm{biph})\right\}^{2-}\left(\mathbf{6}^{2-}\right)$ in $\mathrm{kcal} \cdot \mathrm{mol}^{-1}$.

\begin{tabular}{cccccccc}
\hline $\mathbf{6}^{\mathbf{2}-}$ & Def2-SVP & Def2-TZVPP & $\Delta \mathrm{E}$ & $\Delta \mathrm{Z}_{0}$ & $\Delta \mathrm{E}_{\text {thermal }}$ & $-\mathrm{T} \Delta \mathrm{S}$ & $\Delta \mathrm{G}$ \\
Singlet & 0 & 0 & 0 & 0 & 0 & 0 & 0 \\
Triplet & 13.74 & 0.95 & 14.7 & -1.80 & 0.35 & -0.87 & 12.37 \\
\hline
\end{tabular}

